SAND95-8200

Unlimited Distribution

Printed November 1994

\title{
DYNAMIC RESPONSE OF A TRANSDUCER MOUNTED AT ONE END OF AN ACOUSTICAL CAVITY WHICH IS SUBJECTED TO A SPECIFIED PRESSURE AT THE OPEN END OF THE CAVITY*
}

\author{
G. A. Benedetti \\ and \\ J. Z. Benson \\ Solid Mechanics Department \\ Sandia National Laboratories, Livermore, California
}

\begin{abstract}
The purpose of this report is to develop a mathematical model for a pressure transducer mounted in a fluid filled cavity (a system) and examine the pressure "measurement" error of the cavity and transducer by computing the dynamic response (output pressure) of the system to a specified pressure time history (input pressure). The "measurement" error is determined by comparing the calculated output pressure to the specified input pressure.

The dynamic response of a transducer mounted at one end of a one-dimensional acoustical cavity is determined. The cavity is filled with a compressible isentropic fluid, and the fluid at the open end of the cavity (i.e., the boundary at $x=0$ ) is subjected to a specified uniform axial input pressure. At the other end of the cavity the transducer is represented as a mass, spring, and damper system. Consequently, the boundary condition at $x=\ell$ is also time dependent.

The general solution to the boundary value problem, as well as the steady state solution for periodic excitation, is obtained by integrating a coupled set of ordinary differential equations.
\end{abstract}

*This work was supported by the U. S. Department of Energy under Contract Number

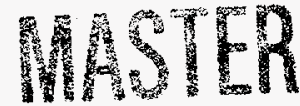
DE-AC04-94AL85000.

DISTRIBUTION OF THIS DOCUMENT IS UNLIMITED

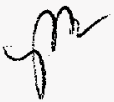


Since the steady state response for harmonic excitation is discussed at length in reference 1, the transient dynamic response is of particular interest in this report. Consequently, pressure shock response spectra are determined for a variety of specified input pressure time histories.

Solutions to the non-dimensional equations of motion are used to generate the nondimensional response spectra. Consequently, the response spectra apply to any one-dimensional acoustic cavity with the same non-dimensional physical system parameters. Numerical examples are provided to illustrate application to practical problems.

Under certain conditions, the time dependent boundary condition associated with the transducer can be replaced with reasonable accuracy by the boundary condition associated with a rigid wall. For this case the equations of motion uncouple and an analytical solution is obtained in the form of an integral which can be evaluated by numerical quadrature.

When the time ratio $\frac{\tau_{1}}{T_{1}}$ is much less than 2.5 , very large pressure measurement errors occur. This results because the highest "measured" input frequency associated with the oscillatory pressure is close to or greater than the first natural frequency of the transducer cavity. Note that the transducer cavity has multiple natural frequencies (refer to reference 1.)

It is also shown that accurate high frequency oscillatory pressure measurements depend not only on the transducer's cavity configuration, frequency response of the measurement system, etc., but can also depend on the diameter of the transducer's sensing element. 


\section{DISCLAIMER}

This report was prepared as an account of work sponsored by an agency of the United States Government. Neither the United States Government nor any agency thereof, nor any of their employees, make any warranty, express or implied, or assumes any legal liability or responsibility for the accuracy, completeness, or usefulness of any information, apparatus, product, or process disclosed, or represents that its use would not infringe privately owned rights. Reference herein to any specific commercial product, process, or service by trade name, trademark, manufacturer, or otherwise does not necessarily constitute or imply its endorsement, recommendation, or favoring by the United States Government or any agency thereof. The views and opinions of authors expressed herein do not necessarily state or reflect those of the United States Government or any agency thereof. 


\section{DISCLAIMER}

Portions of this document may be illegible in electronic image products. Images are produced from the best available original document. 


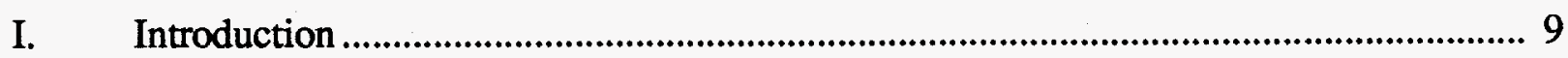

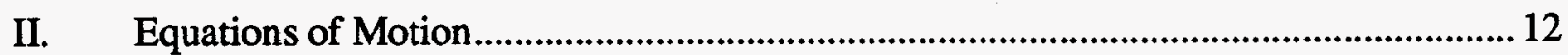

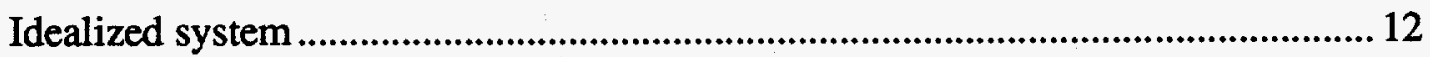

Partial differential equation of motion ................................................................. 13

Boundary conditions ……....................................................................................... 17

III. Complete Solution for the System Dynamic Response ................................................... 18

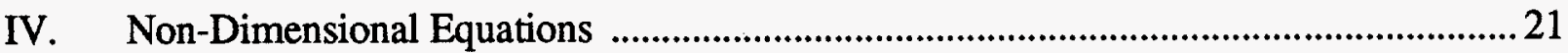

Partial differential equation of motion ..................................................................... 21

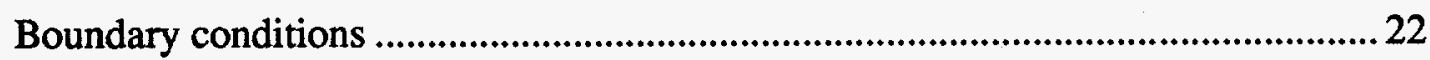

Complete solution for the system dynamic response ................................................ 23

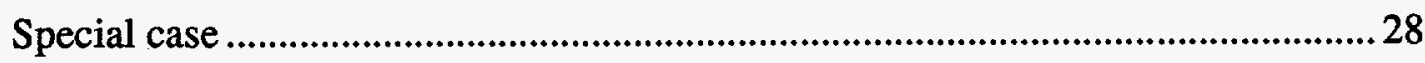

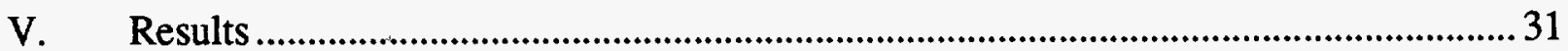

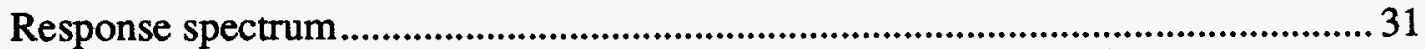

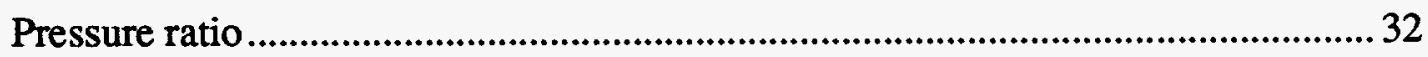

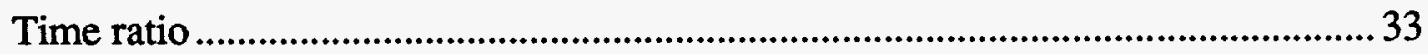

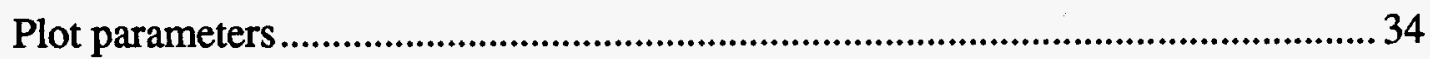

Normalized non-dimensional specified input pressures ...............................................35

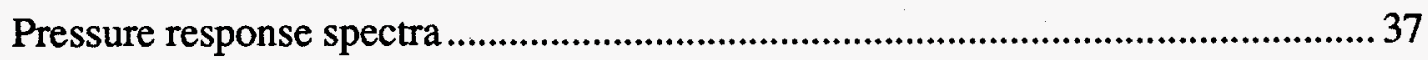

Numerical examples................................................................................................ 71

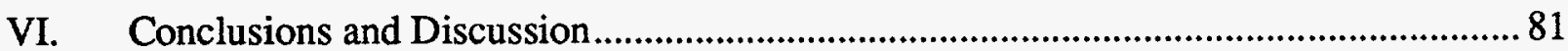

Additional comments regarding measurement error associated with the

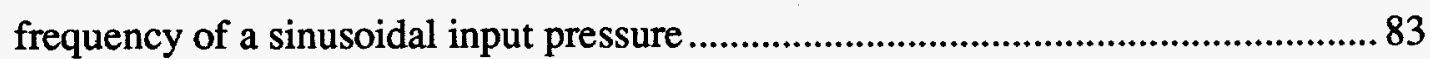

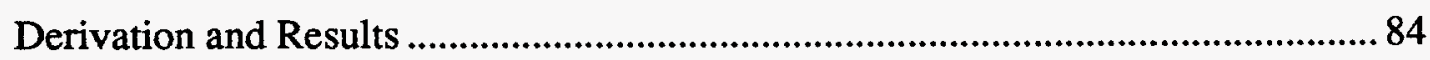

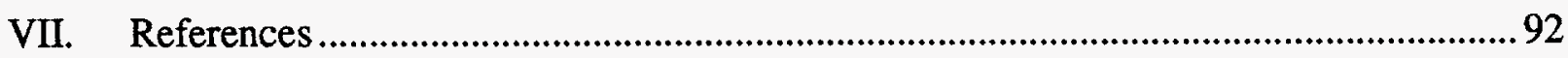

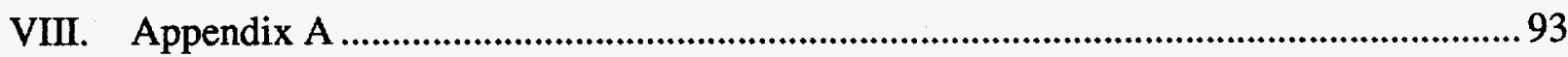

Pressure-time response plots ...................................................................................93 
NOMENCLATURE

A cross-sectional area of tube (or cavity) and piston

$c \quad$ viscous damping coefficient for piston at $x=\ell$

$c_{n} \quad$ viscous damping coefficient for $n^{\text {th }}$ mode for the fluid

$c_{c} \quad$ critical viscous damping coefficient for piston at $x=\ell ; c_{c}=2 m \omega_{\text {nat }}$

$c_{c_{n}} \quad$ critical viscous damping coefficient for $n^{\text {th }}$ mode for compressible fluid; $c_{c_{n}}=2 \rho_{o} \mathrm{~A} \omega_{n}$

$c, c_{o} \quad$ speed of sound; speed of sound at reference conditions in compressible fluid

$c_{p}, c_{v} \quad$ specific heats at constant pressure and constant volume for fluid, respectively

$C_{d n} \quad n^{\text {th }}$ viscous damping coefficient for compressible fluid

$f \quad$ frequency

$J_{1} \quad$ Bessel function of the first kind and first order

$k, k_{f} \quad$ spring constant; ratio of $c_{p}$ to $c_{v}$ for fluid

$\ell \quad$ tube or cavity length at static equilibrium position

$m \quad$ piston mass at right-hand end of cavity

$N \quad$ number of generalized coordinates

$n, r \quad$ indices

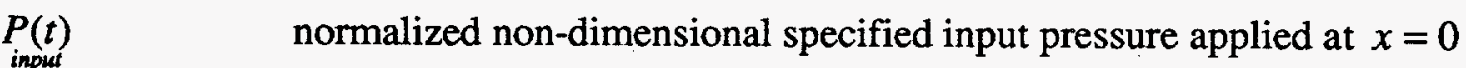

$$
\underset{\text { input }}{P(t)}=\frac{1}{\rho_{o} c_{o}^{2}}\left[p(0, t)-p_{o}\right] \text { and }\left|P_{\text {input }}(\tau)\right|_{\text {max }}=1.0
$$

$\underset{\text { input }}{P(\tau)} \quad$ non-dimensional specified input pressure applied at $\xi=0$

$$
P_{\text {input }}(\tau)=\frac{1}{\rho_{o} c_{o}^{2}}\left[\underset{\text { nor }}{p}(0, \tau)-p_{o}\right]
$$

$\underset{\text { output }}{P(t)} \quad$ non-dimensional output pressure at $x=\ell$

$$
\underset{\text { output }}{P(t)}=\frac{1}{\rho_{o} c_{o}^{2}}\left[p(\ell, t)-p_{o}\right]
$$

$\underset{\text { ouput }}{P(\tau)} \quad$ non-dimensional output pressure at $\xi=1$

$$
\underset{\text { output }}{P(\tau)}=\frac{1}{\rho_{o} c_{o}^{2}}\left[p(1, \tau)-p_{o}\right]
$$

6 
$p$

$p(0, t)$

$\underset{\text { nor }}{p}(0, \tau)$

po

$p(\ell, t)$

$p(1, \tau)$

$\frac{p_{\text {out }}}{p_{\text {in }}}$

$t$

$t_{1}$

$T_{1}$

$\tau$

$\tau_{1}$

$u(x, t)$

$\bar{u}_{c}(n, t)$

$w(\xi, \tau)$

$\bar{w}_{c}(n, \tau)$

$x$

$\xi$

$\alpha$

$\theta$

$\theta_{n}$

$\theta_{\text {nat }}$ cavity pressure

specified input pressure

specified normalized input pressure

cavity pressure at static equilibrium or reference conditions

output pressure at $x=\ell$

output pressure at $\xi=1$

pressure ratio, $\frac{p_{\text {out }}}{p_{\text {in }}}=\frac{\left|\begin{array}{l}P(t) \\ \text { output }\end{array}\right|}{\left|\begin{array}{l}P(t) \\ \text { input }\end{array}\right|}$

time

rise time, pulse duration, half-period of a sinusoid

first natural period of an open-closed cavity, non-dimensional

non-dimensional time, $\tau=\frac{c_{o}}{\ell} t$

rise time, pulse duration, half-period of a sinusoid, non-dimensional

compressible fluid displacement in axial direction

finite Fourier cosine transform of $u(x, t)$

compressible fluid displacement in axial direction, non-dimensional

$$
w(\xi, \tau)=\frac{u(\xi, \tau)}{\ell}
$$

finite Fourier cosine transform of $w(\xi, \tau)$

longitudinal coordinate

non-dimensional longitudinal coordinate, $\xi=\frac{x}{\ell}$

ratio of mass of fluid in cavity to mass of piston located at $x=\ell$

non-dimensional frequency of input forcing function, $\theta=\omega \frac{\ell}{c_{o}}$

$\mathrm{n}^{\text {th }}$ non-dimensional natural frequency for fluid in cavity, $\theta_{n}=\omega_{n} \frac{\ell}{c_{o}}$

non-dimensional natural frequency of transducer, $\theta_{\text {nat }}=\omega_{\text {nat }} \frac{\ell}{c_{0}}$ 
$\lambda$

$\zeta$

$\zeta_{n}$

$\rho, \rho_{o}$

$\omega$

$\omega_{n}$

$\omega_{\text {nd }}$

$\omega_{\text {nat }}$

( )

( )

wave length

position along $x$ axis; damping factor for piston at $x=\ell$ or ratio of viscous damping coefficient to the critical viscous damping coefficient for the piston $n^{\text {th }}$ damping factor for fluid or ratio of the $n^{\text {th }}$ viscous damping coefficient to the critical viscous damping coefficient for the fluid for the $n^{\text {th }}$ mode mass density; mass density at static equilibrium for compressible fluid circular frequency of input forcing function $n^{\text {th }}$ undamped natural circular frequency for fluid in cavity $n^{\text {th }}$ damped natural circular frequency for fluid in cavity undamped natural circular frequency of transducer located at $x=\ell$,

$$
\omega_{\text {nat }}=\left[\frac{k}{m}\right]^{1 / 2}
$$

differentiation with respect to real time, $t$ differentiation with respect to non-dimensional time, $\tau$ 


\section{DYNAMIC RESPONSE OF A TRANSDUCER MOUNTED AT ONE END OF AN ACOUSTICAL CAVITY WHICH IS SUBJECTED TO A SPECIFIED PRESSURE AT THE OPEN END OF THE CAVITY}

\section{Introduction}

The purpose of this report is to develop a mathematical model for a pressure transducer mounted in a fluid filled cavity (a system) and examine the pressure "measurement" error of the cavity and transducer by computing the dynamic response (output pressure) of the system to a specified pressure time history (input pressure). The "measurement" error is determined by comparing the calculated output pressure to the specified input pressure.

The objective of this report is to determine the dynamic response (e.g., displacement and pressure) of a transducer mounted at one end of a one-dimensional acoustical cavity which is filled with a compressible isentropic fluid. The fluid at the open end of the cavity (i.e., the boundary at $x=0$ ) is subjected to a specified uniform axial input pressure (i.e., excitation or input). At the other end of the cavity the transducer is represented as a mass, spring, and damper system. Consequently, the boundary condition at $x=\ell$ is also time dependent and more complicated than the boundary condition at $x=0$.

The general solution to the boundary value problem, as well as the steady state solution for periodic excitation, is obtained by numerically integrating a coupled set of second order ordinary differential equations. Simplifications to the coupled set of equations results in an analytical solution in integral form which is evaluated numerically. The results in reference 1 for harmonic excitation were checked by numerically integrating the appropriate equations in this report.

The results obtained in this report are useful in determining the dynamic response (transient and steady state) of a measurement system such as illustrated in Figure 1. Pressure shock response spectra are determined for a variety of input pressure time functions. These spectra are useful in determining if the magnitude of certain input pressure time histories are significantly altered (i.e., amplified or attenuated, which is a measurement error) by the pressure transducer cavity and/or the transducer. The important and practical case is also examined where the natural frequency of the transducer is much higher than the first natural frequency of the cavity, and the first natural frequency of the cavity is much higher than the highest "measured" input frequency, i.e., $\theta_{\text {nat }} \gg \theta_{1}$ and $\theta_{1} \gg \theta_{\max }$. 
Solutions to the non-dimensional equations of motion are used to generate the nondimensional response spectra. Consequently, the response spectra apply to any one-dimensional acoustic cavity with the same non-dimensional physical system parameters. Numerical examples are provided to illustrate application to practical problems.

Under certain conditions, the time dependent boundary condition associated with the transducer can be replaced with reasonable accuracy by the boundary condition associated with a rigid wall. For this case the equations of motion uncouple and an analytical solution is obtained in the form of an integral which can be evaluated by numerical quadrature.

A major conclusion: for the non-dimensional physical system parameters shown on the response spectra plots and for input pressure time histories which are ramped, triangular, or sinusoidal where the time duration of the input $\tau_{D}$ is an integer multiple of the half period $\tau_{1}$ of the sinusoid (i.e., $\tau_{D}=n \tau_{1}$, where $n=1,2,3, \ldots . N$ ), the maximum measurement error between the maximum output and maximum input pressures (i.e., maximum normalized pressure) is less than or equal to about 20 to $25 \%$ when the time ratio is equal to or greater than 2.5 . That is, when

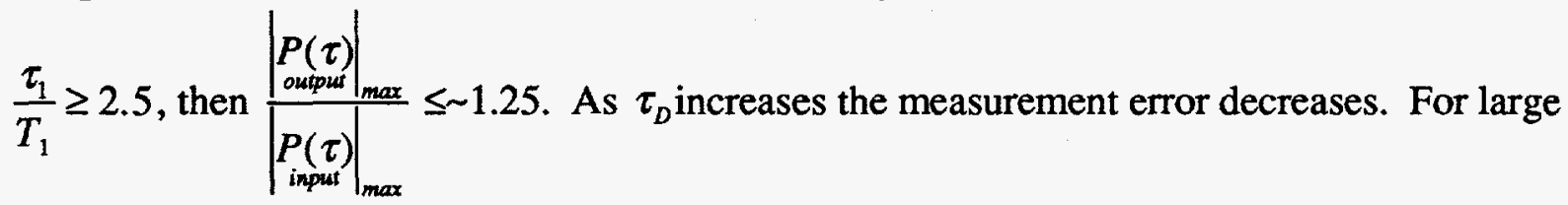
values of $\frac{\tau_{1}}{T_{1}}$ and $\tau_{D}$, the measurement error approaches zero. When the time ratio $\frac{\tau_{1}}{T_{1}}$ is much less than 2.5, very large pressure measurement errors occur. This results because the highest "measured" input frequency associated with the oscillatory pressure is close to or greater than the first natural frequency of the transducer cavity.

References 1 and 2 show that stepped-recessed pressure transducer cavities, which may be grease-filled, are suitable for measuring quasi-static pressure in solid propellant guns and regenerative liquid propellant guns, provided the frequencies contained in the quasi-static pressure pulse are low relative to the fundamental frequency for the empty stepped cavity.

Accurately measured oscillatory pressure-time histories are required to determine the dynamic structural response for projectiles (as well as for guns) and to assess their structural integrity with respect to oscillatory pressure environments in regenerative liquid propellant guns, RLPGs. Unfortunately, grease-filled two diameter cavities or stepped-recessed ports, which have a low fundamental frequency relative to most of the measured frequencies contained in the oscillatory pressures, have been used to measure oscillatory pressures in regenerative liquid propellant guns for many years, and this has resulted in an inaccurate oscillatory pressure data base over a large region of the frequency range of interest. Analysis and laboratory experiments both confirm this. Further, the issue of using grease-filled stepped-recessed transducer ports versus through-hole transducer ports to measure oscillatory pressures has been ongoing for about 
three years and apparently is still not totally resolved. There is ample data which shows that grease-filled stepped-recessed cavities are not suitable for measuring oscillatory pressures over a large region of the frequency range of interest. Therefore, it is strongly recommended that this issue be resolved so that the structural integrity of projectiles (as well as regenerative liquid propellant guns) in the inventory can be accurately assessed when subjected to the oscillatory pressure environment associated with RLPGs [Refs. 1 and 2].

It is also shown that accurate high frequency oscillatory pressure measurements depend not only on the transducer's cavity configuration, frequency response of the measurement system, etc., but can also depend on the diameter of the transducer's sensing element. 


\section{Equations of Motion}

\section{Idealized System}

The idealized system shown in Figure 1 consists of two movable pistons and a compressible fluid surrounded by a straight rigid tube of uniform cross-sectional area. The movable rigid massless piston at the left-hand end of the tube (or cavity) is subjected to a uniform axial pressure specified with respect to time. At the right-hand end of the tube (or cavity) the movable rigid piston, which is connected to a linear spring and a linear viscous damper, responds to pressures (or forces) generated in the compressible fluid by the movable massless piston at the left-hand end of the tube. This system is an approximation for a transducer mounted at the right-hand end of a one-dimensional cavity of length $\ell$ where the transducer is represented by the mass, spring and damper system. The movable massless piston at the lefthand end of the cavity is used to generate displacement and pressure waves in the compressible fluid within the cavity.

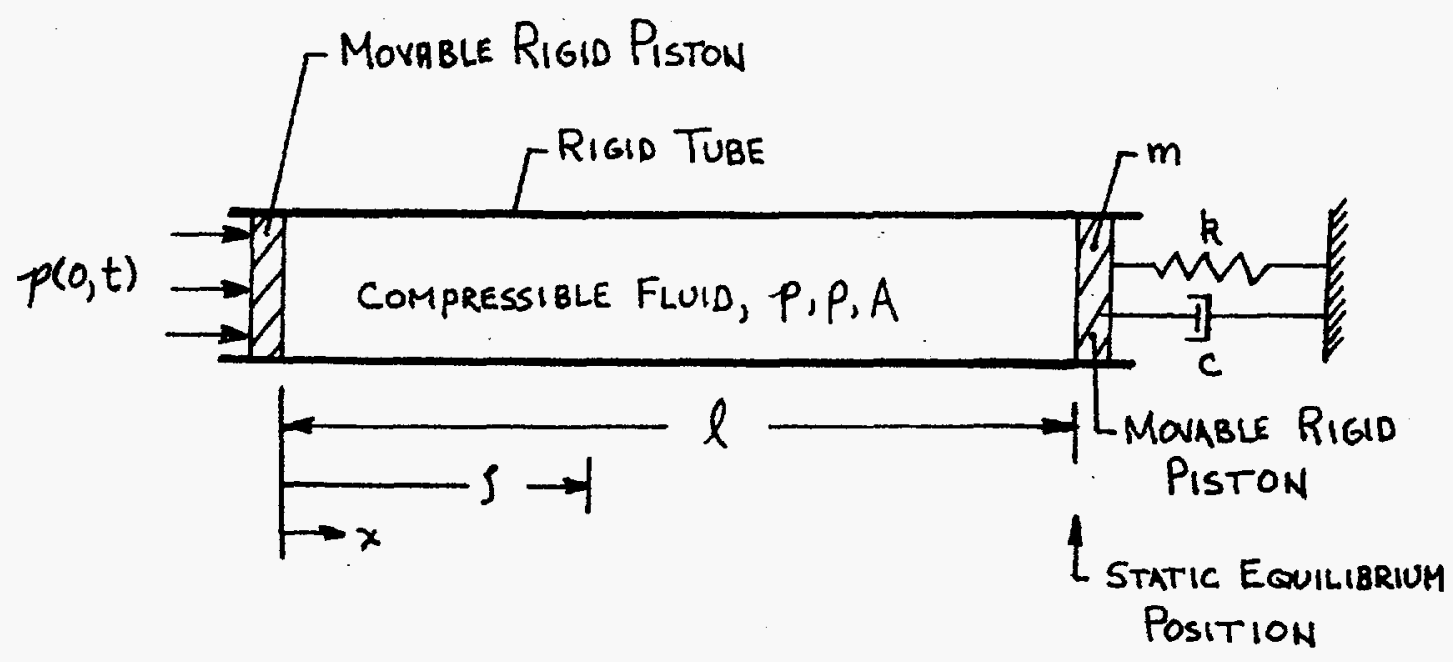

Figure 1. Idealized system. The massless piston at $x=0$ is subjected to a uniform axial pressure specified with respect to time. The piston at $x=\ell$ is supported by a linear spring and linear viscous damper. The rigid tabe is uniform in cross-section and is filled with a compressible fluid. At the static equilibrium position, the density and pressure of the fluid within the tube are $\rho_{o}$ and $p_{o}$ respectively. The fluid properties (e.g., fluid density and pressure) do not vary in any plane perpendicular to the longitudinal or $x$ axis. The isentropic compressible fluid occupies the entire volume of the cavity, which is defined by the rigid tube and the two movable pistons. The massspring-damper system represents a transducer. For the acoustical analysis of a one-dimensional cavity, the wave length, $\lambda=c_{o} / f$ in the cavity fluid is much larger than $\ell, A^{1 / 2}$, and $(\ell A)^{1 / 3}$. That is, $\lambda \gg$ any cavity dimension. Also, changes in pressure are small with respect to the mean pressure. 
The compressible fluid in the tube (or cavity) is assumed to be isentropic and the fluid density and pressure do not vary in any plane perpendicular to the longitudinal or $x$ axis. At the static equilibrium position, the density and pressure of the compressible fluid in the tube are $\rho_{o}$ and $p_{o}$, respectively. The constitutive equation $p=p(\rho)$ for an isentropic fluid is generally nonlinear. In order to linearize the constitutive equation only small changes in the fluid density $\rho$ and pressure $p$ are considered relative to the reference values of $\rho_{o}$ and $p_{o}{ }^{1}$ In addition, it is also assumed that the compressible fluid in the cavity is a perfect gas and that the wave length $\lambda=c_{o} / f$ in the cavity fluid is much greater than any cavity dimension.

The purpose of the subsequent analysis is to determine the pressure dynamic response of the transducer (spring-mass-damper system) in a one-dimensional cavity when the movable massless piston at the left-hand end of the cavity is subjected to a uniform axial pressure specified with respect to time.

\section{Partial Differential Equation of Motion}

Consider the deformed fluid element shown in Figure 2 and recall that the properties of the fluid do not vary in any plane perpendicular to the longitudinal or $x$ axis.

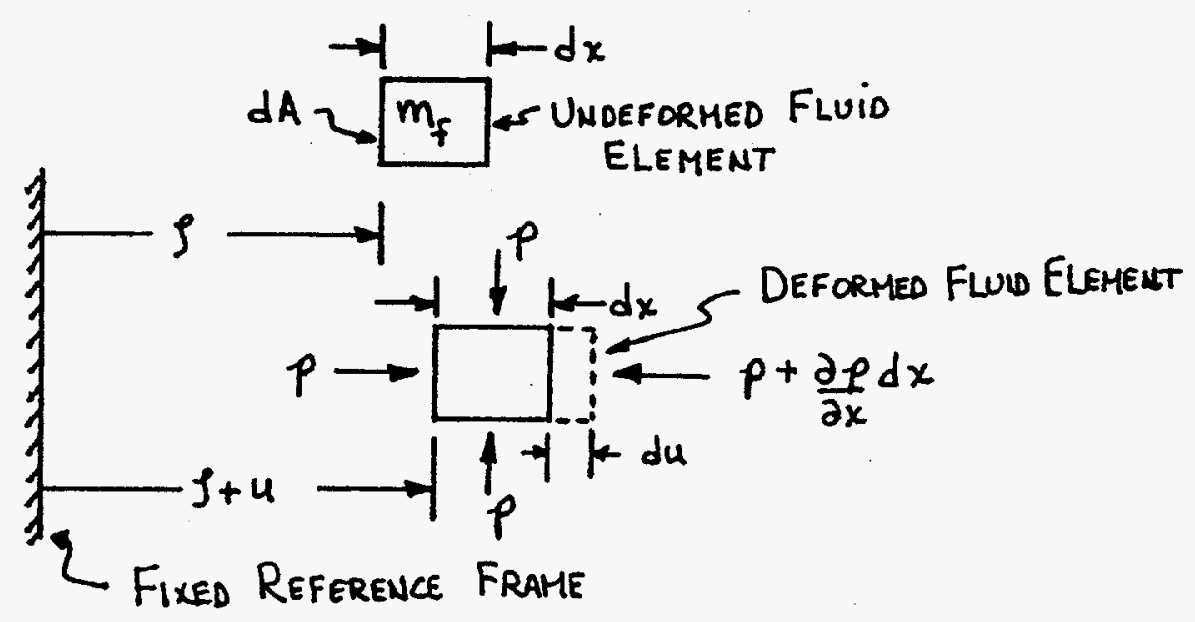

Figure 2. Undeformed and deformed fluid element.

\footnotetext{
${ }^{1}$ This linear approximation to the constitutive equation, as well as linear equations of motion, results in a linearized model and is sometimes called the acoustic approximation.
} 
Applying Newton's second law of motion to the deformed fluid element results in Equation (1).

$$
-\frac{\partial p}{\partial x}-C_{d n} \frac{\partial u}{\partial t}=\rho\left(1+\frac{\partial u}{\partial x}\right) \frac{\partial^{2} u}{\partial t^{2}}
$$

Conservation of mass for the undeformed and deformed fluid element results in Eq. (2).

$$
\rho_{o}=\rho\left(1+\frac{\partial u}{\partial x}\right)
$$

Substituting Eq. (2) into Eq. (1) results in Eq. (3).

$$
-\frac{\partial p}{\partial x}-C_{d n} \frac{\partial u}{\partial t}=\rho_{o} \frac{\partial^{2} u}{\partial t^{2}}
$$

Since the fluid is isentropic, the pressure $p$ can be expressed as a function of the density $\rho$. Also, since the linearized model considers only small changes in the fluid pressure and density with respect to their initial or static equilibrium values $p_{o}$ and $\rho_{o}$, the fluid pressure can be expressed in terms of fluid density by expanding the constitutive equation $p=p(\rho)$ in a Taylor's series about the initial values of $p_{o}$ and $\rho_{o}$ where nonlinear or higher order terms in the series are neglected. The Taylor's series expansion for the fluid pressure is expressed by Eq. (4). ${ }^{2}$

$$
p=p_{o}+\left.\left(\rho-\rho_{o}\right) \frac{d p}{d \rho}\right|_{\rho=\rho_{o}}+\text { H.O. terms }
$$

${ }^{2}$ The Taylor's series, Eq. (4), can be obtained from the power series

$$
p=\sum_{\mathrm{n}=0}^{\mathrm{n}=\infty} a_{\mathrm{n}}\left(\rho-\rho_{o}\right)^{n}
$$

provided $p=p(\rho)$ is continuous and has derivatives. The values of $a_{\mathrm{n}}$ for $n=0,1,2,3$, etc. are: $a_{o}=p\left(\rho_{o}\right)=p_{o} ; a_{1}=\left.\frac{d p}{d \rho}\right|_{\rho=\rho_{o}} ; a_{2}=\left.\frac{1}{2} \frac{d^{2} p}{d \rho^{2}}\right|_{\rho=\rho_{o}} ; a_{3}=\left.\frac{1}{6} \frac{d^{3} p}{d \rho^{3}}\right|_{\rho=\rho_{o}} ;$ itc. 
It is advantageous to express the fluid density $\rho$ in terms of $\rho_{o}$ and $\frac{\partial u}{\partial x}$. Eq. (5) results directly from Eq. (2).

$$
\rho-\rho_{o}=-\rho_{o} \frac{\partial u}{\partial x}\left[\frac{1}{1+\frac{\partial u}{\partial x}}\right]
$$

Note that

$$
\frac{1}{1+\frac{\partial u}{\partial x}}=1-\frac{\partial u}{\partial x}+\left(\frac{\partial u}{\partial x}\right)^{2}-\cdots
$$

Since $\frac{\partial u}{\partial x} \ll<1$, Eq. (5) reduces to Eq. (6). Eq (6) will subsequently be used in Eq. (4).

$$
\rho-\rho_{o}=-\rho_{o} \frac{\partial u}{\partial x}
$$

Since the compressible fluid is isentropic, all processes for the fluid are adiabatic. The fluid is also a perfect gas. Consequently, the equation of state for the gas (fluid) is expressed as Eq. (7).

$$
p\left(\frac{1}{\rho}\right)^{k_{f}}=C
$$

where $k_{f}$ is the ratio of the specific heat at constant pressure to the specific heat at constant volume and $\mathrm{C}$ is a constant. ${ }^{3} \mathrm{Eq}$. (8) results from Eq. (7) by taking the derivative of the pressure $p$ with respect to the density $\rho$.

$$
\frac{d p}{d \rho}=\frac{k_{f} p}{\rho}
$$

For a reversible adiabatic process in a perfect gas, the speed of sound can be expressed by Eq. (9).

3 The specific heats at constant pressure and constant volume are constant for a perfect gas. Also,

$$
C=p_{o}\left(\frac{1}{\rho_{o}}\right)^{k} \text {. }
$$




$$
c=\sqrt{\frac{k_{f} p}{\rho}}
$$

Substituting Eq. (9) into Eq. (8) and evaluating the derivative in Eq. (8) at the reference density $\rho_{o}$ of the compressible fluid (gas) results in Eq. (10)

$$
\left.\frac{d p}{d \rho}\right|_{\rho=\rho_{0}}=c_{o}^{2}
$$

where $c_{o}^{2}=\frac{k_{f} p_{o}}{\rho_{o}} . c_{o}$ represents the speed of sound in the gas at the reference conditions.

Eq. (11) results from incorporating Eqs. (6) and (10) into Eq. (4).

$$
p=p_{o}-\rho_{o} c_{o} \frac{\partial u}{\partial x}
$$

and

$$
\frac{d p}{d x}=-\rho_{o} c_{o}^{2} \frac{\partial^{2} u}{\partial x^{2}}
$$

Utilizing Eq. (12) and Eq. (3) results in Eq. (13), which represents the linearized partial differential equation for the longitudinal motion of the compressible isentropic fluid (perfect gas) within the rigid tube shown in Figure 1.

$$
\begin{aligned}
& \frac{\partial^{2} u}{\partial x^{2}}=\frac{1}{c_{o}^{2}} \frac{\partial^{2} u}{\partial t^{2}}+\frac{C_{d n}}{\rho_{o} c_{o}{ }^{2}} \frac{\partial u}{\partial t} 0 \leq x \leq \ell \\
& t>0
\end{aligned}
$$

The initial conditions for Eq. (13) are expressed by Eq. (14).

$$
\begin{aligned}
& u(x, 0)=0 \\
& \frac{\partial u(x, 0)}{\partial t}=0
\end{aligned}
$$

The time dependent boundary conditions associated with Eq. (13) are discussed in the next section. 


\section{Boundary Conditions}

The specified uniform axial pressure $p(0, t)$ applied to the movable massless piston at $x=0$ is determined from Eq. (11). Consequently, the boundary condition at $x=0$ is a specified function of time given by Eq. (15).

$$
-\frac{\partial u}{\partial x}(0, t)=\underset{\text { input }}{P(t)}
$$

where $\quad \underset{\text { inpua }}{P(t)}=\frac{1}{\rho_{o} c_{o}^{2}}\left[p(0, t)-p_{o}\right]$

Applying Newton's second law of motion in the $x$ direction to the movable piston at $x=\ell$ results in Eq. (16):

$$
\left.\mathrm{Ap}\right|_{x=\ell+u(\ell, t)}=m \frac{d^{2} u}{d t^{2}}(\ell, t)+c \frac{d u}{d t}(\ell, t)+k u(\ell, t)+k \delta
$$

where the force acting on the left-hand face of the piston at $x=\ell+u(\ell, t)$ is Ap $\left.\right|_{x=\ell+u(\ell, t)}$ Utilizing Eq. (11) in Eq. (16) results in Eq. (17) for the boundary condition at $x=\ell$.

$$
m \frac{d^{2} u(\ell, t)}{d t^{2}}+c \frac{d u(\ell, t)}{d t}+k u(\ell, t)+\rho_{o} c_{o}^{2} \mathrm{~A} \frac{\partial u(\ell, t)}{\partial x}=0
$$

Note that $u(\ell, t)$ is measured with respect to the static equilibrium position of the spring, that $A p_{o}=k \delta$ and that $\frac{u(\ell, t)}{\ell} \ll 1$.

Eqs. (15) and (17) represent the time dependent boundary conditions at $x=0$ and $x=\ell$, respectively. 


\section{Complete Solution for the System Dynamic Response}

Because of the nature of the boundary conditions, it is convenient to transform Eq. (13) by using a finite Fourier cosine transform:

$$
\bar{u}_{c}(n, t)=\int_{x=0}^{x=\ell} u(x, t) \cos \frac{\mathrm{n} \pi}{\ell} x d x
$$

where the inverse transform is expressed by Eq. (19) 4

$$
u(x, t)=\frac{1}{\ell} \bar{u}_{c}(0, t)+\frac{2}{\ell} \sum_{n=1}^{n=\infty} \bar{u}_{c}(n, t) \cos \frac{n \pi}{\ell} x
$$

Transforming Eq. (13) results in the following ordinary differential equation:

$$
\left[\frac{\partial u}{\partial x}(x, t) \cos \frac{n \pi}{\ell} x+\frac{n \pi}{\ell} u(x, t) \sin \frac{n \pi}{\ell} x\right]_{x=0}^{x=\ell}-\left(\frac{n \pi}{\ell}\right)^{2} \bar{u}_{c}(n, t)=\frac{1}{c_{0}^{2}} \ddot{\bar{u}}_{c}(n, t)+\frac{C_{d n}}{\rho_{o} c_{o}^{2}} \dot{\bar{u}}_{c}(n, t)
$$

where $n=0,1,2,3, \ldots N$

and from Eq. (15)

$$
-\frac{\partial u}{\partial x}(0, t)=\frac{1}{\rho_{o} c_{o}^{2}}\left[p(0, t)-p_{o}\right]
$$

Eq. (22) results directly from Eq. (17). Note that the right side of Eq. (22) is evaluated at $x=\ell$.

$$
\frac{\partial u}{\partial x}(\ell, t)=-\frac{1}{\rho_{o} c_{o}^{2} A}[m \ddot{u}(x, t)+c \dot{u}(x, t)+k u(x, t)]_{x=\ell}
$$

Utilizing Eq. (19) and its appropriate derivatives with respect to time in Eq. (22) results in Eq. (23).

${ }^{4}$ Recall that $0 \leq x \leq \ell$ and that within this range the function $u(x, t)$ must satisfy Dirichlet's conditions. At any point of discontinuity, the left side of Eq. (19) should be replaced by the average,

$$
\frac{1}{2}[u(x+0, t)+u(x-0, t)]
$$




$$
\begin{aligned}
\frac{\partial u(\ell, t)}{\partial x}= & -\frac{1}{\rho_{o} c_{0}^{2} \mathrm{~A} \ell}\left[m \ddot{\bar{u}}_{c}(0, t)+c \dot{\bar{u}}_{c}(0, t)+k \bar{u}_{c}(0, t)\right] \\
& -\frac{1}{\rho_{o} c_{o}^{2} \mathrm{~A}} \frac{2}{\ell} \sum_{r=1}^{r=\infty}(-1)^{r}\left[m \ddot{\bar{u}}_{c}(r, t)+c \dot{\bar{u}}_{c}(r, t)+k \bar{u}_{c}(r, t)\right]
\end{aligned}
$$

where $n$ and $\cos n \pi$ are replaced by $r$ and $(-1)^{r}$, respectively.

Substituting Eqs. (15) and (23) into Eq. (20) and simplifying, results in a set of coupled ordinary differential equations for $\bar{u}_{c}(n, t)$ :

$$
\begin{aligned}
& \ddot{\bar{u}}_{c}(n, t)+\frac{(-1)^{n}}{\alpha} \ddot{\bar{u}}_{c}(0, t)+(-1)^{n} \frac{2}{\alpha} \sum_{r=1}^{r=N}(-1)^{r} \ddot{\bar{u}}_{c}(r, t) \\
& +2 \zeta_{n} n \pi \dot{\bar{u}}_{c}(n, t)+(-1)^{n} \frac{2 \zeta \theta_{\text {nat }}}{\alpha} \dot{\bar{u}}_{c}(0, t)+(-1)^{n} \frac{4 \zeta \theta_{n a t}}{\alpha} \sum_{r=1}^{r=N}(-1)^{r} \dot{\bar{u}}_{c}(r, t) \\
& +(n \pi)^{2} \bar{u}_{c}(n, t)+(-1)^{n} \frac{\theta_{n a t}^{2}}{\alpha} \bar{u}_{c}(0, t)+(-1)^{n} \frac{2 \theta_{\text {nat }}^{2}}{\alpha} \sum_{r=1}^{r=N}(-1)^{r} \bar{u}_{c}(r, t)=c_{o}^{2} P_{\text {input }}(t) \\
& n=0,1,2,3, \ldots N
\end{aligned}
$$

where

$$
\begin{aligned}
& \alpha=\frac{\rho_{o} \ell \mathrm{A}}{m} \\
& \zeta_{n}=\frac{c_{n}}{c_{c_{n}}} \\
& c_{c_{n}}=2 \rho_{o} \mathrm{~A} \omega_{n} \\
& \zeta=\frac{c}{c_{c}} \\
& c_{c}=2 m \omega_{\text {nat }} \\
& \omega_{\text {nat }}=\left[\frac{k}{m}\right]^{1 / 2} \\
& \omega_{n}=n \pi \frac{c_{o}}{\ell} \\
& \theta_{\text {nat }}=\omega_{\text {nat }} \frac{\ell}{c_{o}} \\
& P_{\text {input }}(t)=\frac{1}{\rho_{o} c_{o}^{2}}\left[p(0, t)-p_{o}\right]
\end{aligned}
$$




$$
\begin{aligned}
& c_{o}^{2}=\frac{k_{f} p_{o}}{\rho_{o}} \\
& k_{f}=\frac{c_{p}}{c_{v}} \\
& C_{d n}=2 \zeta_{n} \omega_{n} \rho_{o}
\end{aligned}
$$

and $\underset{\text { input }}{P(t)}$ is a non-dimensional specified input pressure applied at $x=0$. Note that $\underset{\text { input }}{P(t)}$ is determined from $p(0, t)$ which is the specified input pressure applied to the face of the massless piston located at $x=0$.

The transformed initial conditions are expressed by Eq. (26) and are obtained from Eqs. (14) and (18).

$$
\begin{aligned}
& \bar{u}_{c}(n, 0)=0 \\
& \dot{\bar{u}}_{c}(n, 0)=0
\end{aligned} \quad n=0,1,2,3, \ldots \mathrm{N}
$$

The longitudinal displacement $u(x, t)$ of the isentropic compressible fluid (gas) is obtained by solving Eqs. (24) for $\bar{u}_{c}(n, t) n=0,1,2,3, \ldots . \mathrm{N}$ and then applying Eq. (19) for the inverse transform. ${ }^{5}$ The output pressure $p(\ell, t)$ is obtained by using Eq. (23) in conjunction with Eq. (11) applied at the boundary $x=\ell$.

It is convenient to non-dimensionalize Eqs. (11), (13), (14), (17), (18), (19), (22), (23), and (24). This is discussed in the next section.

5 The mass, damping and stiffness matrices represented by Eqs. (24) are not symmetric nor are they skew symmetric. Note that Eq. (24) in reference 1 is symmetric. Eq. (24) from reference 1 is given below.

$$
\begin{aligned}
& \ddot{\bar{u}}_{s}(n, t)+\frac{2}{\alpha}(-1)^{n-1} \sum_{r=1}^{r=N}(-1)^{r-1} \ddot{\bar{u}}_{s}(r, t)+2 \zeta_{n} \omega_{n}\left[\dot{\bar{u}}_{s}(n, t)+\frac{2}{\alpha} \frac{\zeta}{\zeta_{n}} \frac{\omega_{n a t}}{\omega_{n}}(-1)^{n-1} \sum_{r=1}^{r=N}(-1)^{r-1} \dot{\bar{u}}_{s}(r, t)\right] \\
& +\omega_{n}^{2}\left[\bar{u}_{s}(n, t)+\frac{2}{\alpha} \frac{\omega_{n a t}^{2}}{\omega_{n}^{2}}(-1)^{n-1} \sum_{r=1}^{r=N}(-1)^{r-1} \bar{u}_{s}(r, t)\right]=\omega_{n} c_{o} U(t), \quad n=1,2,3, \ldots N
\end{aligned}
$$




\section{Non-Dimensional Equations}

\section{Partial Differential Equation of Motion}

$\xi$ and $\tau$ are non-dimensional space and time coordinates and are defined by Eqs. (27) and (28).

$$
\begin{aligned}
& \xi=\frac{x}{\ell} \\
& \tau=\frac{c_{o}}{\ell} t
\end{aligned}
$$

$w(\xi, \tau)$ is a non-dimensional fluid particle axial displacement and is defined by Eq. (29).

$$
w(\xi, \tau)=\frac{u(\xi, \tau)}{\ell}
$$

Utilizing Eqs. (27), (28), and (29) in Eq. (13) results in Eq. (30) for the partial differential equation of motion where $w=w(\xi, \tau)$.

$$
\begin{aligned}
& \frac{\partial^{2} w}{\partial \xi^{2}}=\frac{\partial^{2} w}{\partial \tau^{2}}+\frac{C_{d n} \ell}{\rho_{o} c_{o}} \frac{\partial w}{\partial \tau} \\
& 0 \leq \xi \leq 1 \\
& \tau>0
\end{aligned}
$$

The initial conditions for Eq. (30) are expressed by Eq. (31)

$$
\begin{aligned}
& w(\xi, 0)=0 \\
& \frac{\partial w}{\partial \tau}(\xi, 0)=0
\end{aligned}
$$

The time-dependent non-dimensional boundary conditions associated with Eq. (30) are discussed in the next section. 


\section{Boundary Conditions}

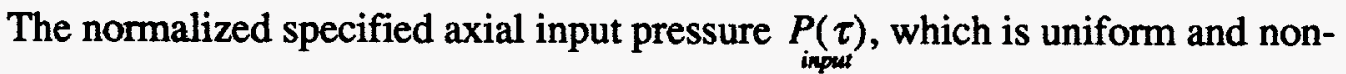
dimensional, is applied to the movable massless piston at $\xi=0$ and the boundary condition is determined from Eq. (11) by utilizing Eqs. (27), (28), and (29).

$$
-\frac{\partial w}{\partial \xi}(0, \tau)=P(\tau)
$$

where $\quad \underset{\text { inpus }}{P(\tau)}=\frac{1}{\rho_{o} c_{0}^{2}}\left[\underset{\text { nor }}{p}(0, \tau)-p_{o}\right]$

and $\quad \underset{\text { oor }}{p}(0, \tau)=\rho_{o} c_{o}^{2}\left[\frac{p_{o}}{\rho_{o} c_{o}^{2}}+\frac{p\left(0, \frac{c_{o}}{\ell} t\right)}{\left|p\left(0, \frac{c_{o}}{\ell} t\right)\right|_{\max }}\right]$

The boundary condition at $\xi=1$ is determined from Eq. (17) and is expressed by Eq. (33a).

$$
\frac{\partial w}{\partial \xi}(1, \tau)=-\frac{1}{\alpha}\left[w^{\prime \prime}(\xi, \tau)+2 \zeta \theta_{\text {nat }} w^{\prime}(\xi, \tau)+\theta_{\text {nat }}^{2} w(\xi, \tau)\right]_{\xi=1}
$$

where 


$$
\begin{aligned}
& \alpha=\frac{\rho_{o} \ell A}{\mathrm{~m}} \\
& \theta_{\text {nat }}=\omega_{\text {nat }} \frac{\ell}{\mathrm{c}_{\mathrm{o}}} \\
& \zeta=\frac{c}{c_{c}} \\
& c_{c}=2 m \omega_{\text {nat }} \\
& \omega_{\text {nat }}=\left[\frac{k}{m}\right]^{1 / 2} \\
& \xi=\frac{x}{\ell} \\
& \tau=\frac{c_{o} t}{\ell} \\
& \frac{\partial()^{\prime}}{\partial \tau}=()^{\prime} \\
& \frac{\partial()}{\partial t}=()
\end{aligned}
$$

Eqs. (32) and (33) represent the non-dimensional time dependent boundary conditions at $\xi=0$ and $\xi=1$, respectively.

\section{Complete Solution for the System Dynamic Response}

Due to the nature of the boundary conditions, it is convenient to transform Eq. (30) by using a finite Fourier cosine transform:

$$
\bar{w}_{c}(n, \tau)=\int_{\xi=0}^{\xi=1} w(\xi, \tau) \cos n \pi \xi d \xi
$$

where the inverse transform is expressed by Eq. (35). ${ }^{6}$

6 Recall that $0 \leq \xi \leq 1$ and that within this range the function $w(\xi, \tau)$ must satisfy Dirichlet's conditions. At any point of discontinuity, the left side of Eq. (35) should be replaced by the average, $\frac{1}{2}[w(\xi+0, \tau)+w(\xi-0, \tau)]$. 


$$
w(\xi, \tau)=\bar{w}_{c}(0, \tau)+2 \sum_{n=1}^{n=\infty} \bar{w}_{c}(n, \tau) \cos n \pi \xi
$$

Eq. (35) is determined by expanding $w(\xi, \tau)$ in a Fourier cosine series [Eq. (36)] and substituting Eq. (36) into Eq. (34)

$$
w(\xi, \tau)=\sum_{r=0}^{r=\infty} a_{r}(\tau) \cos r \pi \xi
$$

and integrating. This results in the following:

$$
\begin{array}{ll}
a_{o}(\tau)=\bar{w}_{c}(0, \tau) & \text { for } r=n=0 \\
a_{n}(\tau)=2 \bar{w}_{c}(n, \tau) & \text { for } r=n \\
& \text { and } r \neq 0
\end{array}
$$

Substituting Eq. (37) into Eq. (36) results in Eq. (35).

Transforming Eq. (30) results in the following ordinary differential equation. Note that integration by parts is necessary to evaluate $\frac{\partial^{2} w}{\partial \xi^{2}}(\xi, \tau)$.

$$
\begin{aligned}
& {\left[\frac{\partial w}{\partial \xi}(\xi, \tau) \cos n \pi \xi+(n \pi) w(\xi, \tau) \sin n \pi \xi\right]_{\xi=0}^{\xi=1}-(n \pi)^{2} \bar{w}_{c}(n, \tau)=} \\
& \bar{w}_{c}^{\prime \prime}(n, \tau)+\frac{C_{d n} \ell}{\rho_{o} c_{o}} \bar{w}_{c}^{\prime}(n, \tau) \\
& n=0,1,2,3, \ldots . . N
\end{aligned}
$$

Eq. (38) reduces to Eq. (39) upon substitution of the limits of integration.

$$
\begin{array}{cc}
\bar{w}_{c}^{\prime \prime}(n, t)+\frac{C_{d_{n}} \ell}{\rho_{o} c_{o}} \bar{w}_{c}^{\prime}(n, t)+(n \pi)^{2} \bar{w}_{c}(n, \tau)-\left.\frac{\partial w}{\partial \xi}(\xi, \tau)\right|_{\xi=1} \cos n \pi= \\
-\left.\frac{\partial w}{\partial \xi}(\xi, \tau)\right|_{\xi=0} & n=0,1,2,3, \ldots . . N
\end{array}
$$

Recall that $\bar{w}_{c}(n, \tau)$ is the finite Fourier cosine transform of $w(\xi, \tau)$.

Utilizing Eq. (35) and its appropriate derivatives with respect to $\tau$ in Eq. (33a) results in Eq. (40) which represents the transformed time dependent boundary condition at $\xi=1$. 


$$
\begin{gathered}
-\left.\frac{\partial w}{\partial \xi}(\xi, \tau)\right|_{\xi=1}=\frac{1}{\alpha} \bar{w}_{c}^{\prime \prime}(0, \tau)+\frac{2}{\alpha} \sum_{r=1}^{r=N}(-1)^{r} \bar{w}_{c}^{\prime \prime}(r, \tau) \\
+\frac{2 \zeta \theta_{\text {nat }}}{\alpha} \bar{w}_{c}^{\prime}(0, \tau)+\frac{4 \zeta \theta_{\text {nat }}^{r=N}}{\alpha} \sum_{r=1}^{r=N}(-1)^{r} \bar{w}_{c}^{\prime}(r, \tau) \\
+\frac{\theta_{n a t}^{2}}{\alpha} \bar{w}_{c}(0, \tau)+\frac{2 \theta_{\text {nat }}^{2} \sum_{r=1}^{r=N}(-1)^{r} \bar{w}_{c}(r, \tau)}{\alpha}
\end{gathered}
$$

where $n$ and $\cos n \pi$ are replaced with $r$ and $(-1)^{r}$, respectively.

Substituting Eqs. (32a) and (40) into Eq. (39) and simplifying results in a set of coupled ordinary differential equations for $\bar{w}_{c}(n, \tau)$ :

$$
\begin{aligned}
& \bar{w}_{c}^{\prime \prime}(n, \tau)+\frac{(-1)^{n}}{\alpha} \bar{w}_{c}^{\prime \prime}(0, \tau)+(-1)^{n} \frac{2}{\alpha} \sum_{r=1}^{r=N}(-1)^{r} \bar{w}_{c}^{\prime \prime}(r, \tau) \\
& +2 \zeta_{n} n \pi \bar{w}_{c}^{\prime}(n, \tau)+(-1)^{n} \frac{2 \zeta \theta_{\text {nat }}}{\alpha} \bar{w}_{c}^{\prime}(0, \tau)+(-1)^{n} \frac{4 \zeta \theta_{n a t}}{\alpha} \sum_{r=1}^{r=N}(-1)^{r} \bar{w}_{c}^{\prime}(r, \tau) \\
& +(n \pi)^{2} \bar{w}_{c}(n, \tau)+(-1)^{n} \frac{\theta^{2}{ }_{n a t}}{\alpha} \bar{w}_{c}(0, \tau)+(-1)^{n} \frac{2 \theta^{2}{ }_{n a t}}{\alpha} \sum_{r=1}^{r=N}(-1)^{r} \bar{w}_{c}(r, \tau)=P_{i n p u t} \\
& n=0,1,2,3, \ldots . . N
\end{aligned}
$$


where

$$
\begin{aligned}
& \alpha=\frac{\rho_{o} \ell A}{m} \\
& \zeta_{n}=\frac{c_{n}}{c_{c_{n}}} \\
& \frac{c_{n}}{c_{c_{n}}}=2 \rho_{o} A \omega_{n} \\
& \zeta=\frac{c}{c_{n}} \\
& c_{c}=2 m \omega_{\text {nat }} \\
& \omega_{\text {nat }}=\left[\frac{k}{m}\right]^{1 / 2} \\
& \omega_{n}=n \pi \frac{c_{o}}{\ell} \\
& \theta_{n}=\omega_{n} \frac{\ell}{c_{o}}=n \pi \\
& \theta_{\text {nat }}=\omega_{\text {nat }} \frac{\ell}{c_{o}} \\
& \tau=\frac{c_{o}}{\ell} t \\
& \xi=\frac{x}{\ell} \\
& c_{o}^{2}=\frac{k_{f} p_{o}}{\rho_{o}} \\
& \frac{\partial()}{\partial \tau}=()^{\prime} \\
& k_{f}=\frac{c_{p}}{c_{v}} \\
& C_{d n}=2 \zeta_{n} \omega_{n} \rho_{o} \\
& P(\tau)=\frac{1}{\rho_{o} c_{o}^{2}}\left[\underset{\text { inpor }}{p}(0, \tau)-p_{o}\right] \\
& \underset{\text { nor }}{p}(0, \tau)=\rho_{o} c_{o}^{2}\left[\frac{p_{o}}{\rho_{o} c_{o}^{2}}+\frac{p\left(0, \frac{c_{o}}{\ell} t\right)}{\left|p\left(0, \frac{c_{o}}{\ell} t\right)\right|_{\max }}\right]
\end{aligned}
$$


and $\underset{\text { input }}{P(\tau)}$ is a specified function of time. Recall that $\underset{\text { input }}{ }(\tau)$ is a normalized non-dimensional specified input pressure (uniform axial pressure) applied to the massless piston located at $\xi=0$. $\underset{n o r}{p}(0, \tau)$ is a specified normalized input pressure applied at $\xi=0$.

The transformed initial conditions are expressed by Eq. (42) and are obtained from Eq. (31).

$$
\begin{aligned}
& \bar{w}_{c}(n, 0)=0 \\
& \bar{w}_{c}^{\prime}(n, 0)=0
\end{aligned}
$$$$
\mathrm{n}=0,1,2,3, \ldots . \mathrm{N}
$$

The non-dimensional longitudinal displacement $w(\xi, \tau)$ of the isentropic compressible fluid (gas) is obtained by solving Eqs. (41) for $\bar{w}_{c}(n, \tau), n=0,1,2,3, \ldots N$, and then applying Eq. (35) for the inverse transform. ${ }^{7}$ The pressure in the fluid is obtained from Eq. (43)

$$
P(\xi, \tau)=-\frac{\partial w}{\partial \xi}(\xi, \tau)
$$

where

$$
-\frac{\partial w}{\partial \xi}(\xi, \tau)=2 \pi \sum_{n=1}^{n=\infty} n \bar{w}_{c}(n, \tau) \sin n \pi \xi
$$

and

$$
P(\xi, \tau)=\frac{1}{\rho_{o} c_{0}^{2}}\left[p(\xi, \tau)-p_{o}\right]
$$

The normalized non-dimensional input pressure $P(\tau)$ in specified with respect to $\tau$ and acts on the massless piston at $\xi=0$. Refer to Eq. (32b). The non-dimensional output pressure $\underset{\text { ouppur }}{P(\tau)}$ acting on the transducer piston located at $\xi=1$ is obtained from Eq. (44). That is,

$$
\underset{\text { oupput }}{P(\tau)}=-\frac{\partial w}{\partial \xi}(1, \tau)
$$

\footnotetext{
7 The mass, damping, and stiffness matrices represented by Eqs. (41) are not symmetric nor are they skew symmetric.
} 
where $-\frac{\partial w}{\partial \xi}(1, \tau)$ is expressed by Eq. (40)

and

$$
\underset{\text { oupput }}{P(\tau)}=\frac{1}{\rho_{o} c_{0}^{2}}\left[p(1, \tau)-p_{o}\right]
$$

Note that once $P(\tau)$ is determined, the output pressure $p(1, \tau)$ with respect to non-dimensional time $\tau$ can be obtained from Eq. (45). Also, note that $p(1, \tau)$ can be changed to $p(1, t)$ by multiplying each non-dimensional time point $\tau_{\mathrm{i}}$ associated with $p(1, \tau)$ by $\frac{\ell}{c_{o}}$ where $\ell$ and $c_{o}$ depend on the particular problem under consideration. Recall that $\underset{n o r}{p}(0, \tau)$ is a specified normalized input pressure.

\section{Special Case}

Since $\alpha<<1$ in most practical problems, the off-diagonal terms in the mass, damping and stiffness matrices in Eq. (41) can be nearly as large as some of the terms on the main diagonal. Consequently, Eqs. (41) can be highly coupled.

In most practical problems the transducer's first natural frequency is much greater than the highest input frequency $\theta_{\text {nat }} \gg \theta_{\max }$. For this case the displacement boundary condition at $\xi=1$ can be considered as fixed; that is, $w(1, \tau)=0$. (Also, in most practical problems the first natural frequency of an open-closed cavity is much greater than the highest input frequency $\theta_{1} \gg \theta_{\max }$.) Utilizing the boundary condition $w(\xi, \tau)=0$ at $\xi=1$ results in Eq. (46) which greatly simplifies Eqs. (41) and (40). Note that Eq. (46) is an approximation for Eq. (41) and that in a large region of the parameter space the approximation is quite good. (Refer to Section V. Also, refer to Appendix A.)

$$
\bar{w}_{c}^{\prime \prime}(n, \tau)+2 \zeta_{n}(2 n-1) \frac{\pi}{2} \bar{w}_{c}^{\prime}(n, \tau)+\left[(2 n-1) \frac{\pi}{2}\right]^{2} \bar{w}_{c}(n, \tau)=P_{\text {input }}(\tau) \quad n=1,2,3, \ldots . . N
$$

where the initial conditions are given by Eq. (42) for $n=1,2,3, \ldots . N$. The non-dimensional longitudinal displacement $w(\xi, \tau)$ of the isentropic compressible fluid (gas) is obtained by 
solving Eq. (46) for $\bar{w}_{c}(n, \tau), n=1,2,3, \ldots N$, and then applying Eq. (47) for the inverse transform.

$$
w(\xi, \tau)=2 \sum_{n=1}^{n=N} \bar{w}_{c}(n, \tau) \cos (2 n-1) \frac{\pi}{2} \xi
$$

and

$$
-\frac{\partial w}{\partial \xi}(\xi, \tau)=\pi \sum_{n=1}^{n=N}(2 n-1) \bar{w}_{c}(n, \tau) \sin (2 n-1) \frac{\pi}{2} \xi
$$

The non-dimensional pressure in the fluid is obtained from Eq. (49)

$$
P(\xi, \tau)=-\frac{\partial w}{\partial \xi}(\xi, \tau)
$$

where

$$
P(\xi, \tau)=\frac{1}{\rho_{o} c_{o}^{2}}\left[p(\xi, \tau)-p_{o}\right]
$$

The normalized non-dimensional input pressure $\underset{\text { input }}{P(\tau)}$ is specified with respect to $\tau$ and acts on the massless piston located at $\xi=0$. Refer to Eq. (32b). The non-dimensional output pressure $P(\tau)$ acting on the transducer piston located at $\xi=1$ is obtained from Eq. (51). That is,

$$
\underset{\text { oupput }}{P(\tau)}=-\frac{\partial w}{\partial \xi}(1, \tau)
$$

where

$$
\underset{\text { ouput }}{P(\tau)}=\frac{1}{\rho_{o} c_{o}^{2}}\left[p(1, \tau)-p_{o}\right]
$$

and $-\frac{\partial w}{\partial \xi}(1, \tau)$ is obtained from Eq. (48) and expressed by Eq. (53). Note that $P(1, \tau)=\underset{\text { oupput }}{P(\tau)}$. 


$$
-\frac{\partial w}{\partial \xi}(1, \tau)=\pi \sum_{n=1}^{n=N}(2 n-1)(-1)^{n-1} \bar{w}_{c}(n, \tau)
$$

An alternative to numerically integrating Eqs. (46) to determine $\bar{w}_{c}(n, \tau)$ for $n=1,2,3, \ldots N$, is an analytical solution for Eq. (46) which is expressed in the form of a convolution integral given by Eq. (54) for zero initial conditions.

$$
\bar{w}_{c}(n, \tau)=\frac{1}{\theta_{n d}} \int_{t=0}^{t=\tau} P_{i n p u t}(t) e^{-\zeta_{n} \theta_{n}(\tau-t)} \sin \theta_{n d}(\tau-t) d t
$$

where

$$
\begin{aligned}
& \theta_{n d}=\theta_{n} \sqrt{1-\zeta_{n}^{2}} \\
& \theta_{n}=(2 n-1) \frac{\pi}{2}
\end{aligned}
$$

and $t$ is a dummy variable and $n=1,2,3, \ldots N$. 


\section{RESULTS}

\section{Response Spectrum}

A shock represents a sudden application of a force or other form of disruption which results in a transient response of a system. The maximum value of the response is a good measure of the severity of the shock and is, of course, dependent upon the dynamic characteristics of the system. In order to categorize all types of shock excitations, a single degree-of-freedom oscillator (spring- mass system) is usually chosen as a standard system. However, the pressure shock response spectra presented in this section are for an $\mathrm{N}$ degree-of-freedom system (e.g., the pressure transducer cavity).

The concept of the response spectrum is useful in design. A response spectrum is a plot of the maximum peak response of the system as a function of the first natural frequency of the system. Different types of shock excitations will then result in different response spectra.

Since a point on the response spectrum is determined from a single point (the maximum response) on the time response curve for the system (refer to Appendix A), it does not uniquely define the shock input. In fact, it is possible for two different shock excitations to have very similar response spectra. In spite of this limitation, the response spectrum is a useful concept that is extensively used.

The non-dimensional pressure shock response spectra presented in this section are for the pressure transducer cavity (an $\mathrm{N}$ degree-of-freedom system) shown in Figure 1 for various normalized non-dimensional specified input pressures $P_{\text {input }}(\tau)$ applied to the face of the massless piston located at $\xi=0$. Note that the maximum value of $P_{\text {input }}(\tau)$ is unity. Two types of boundary conditions are considered at the end of the cavity located at $\xi=1$ (or $x=\ell$ ).

The first type of boundary condition at $\xi=1$ is a spring-mass-damper system which is used to approximate the pressure transducer. The transformed non-dimensional equation of motion for this system is expressed by Eq. (41). On the plots, the first type of boundary condition is denoted as a generalized boundary condition.

The second type of boundary condition at $\xi=1$ is a fixed end (or rigid wall) and is incapable of any displacement (i.e., $w(1, \tau)=0$ ). This boundary condition is a good approximation for the transducer provided the transducer's first natural frequency is much greater than the highest input frequency $\theta_{\text {nat }} \gg \theta_{\max }$ where $\theta_{\max }$ is the highest input frequency in the "forcing function." The transformed non-dimensional equation of motion of this system is expressed by Eq. (46) or Eq. (54). On the plots the second type of boundary condition is denoted as a simplified boundary condition. 
The solutions to Eq. (46) by numerical integration and to Eq. (54) by numerical quadrature give identical results; however, numerical quadrature was found to be a much longer process. Consequently, the solutions to Eqs. (41) and (46) were obtained by numerical integration.

For most of the pressure shock response spectra plotted in this section, the non-dimensional system parameters are chosen for the important and practical situation where $\alpha<1$, $\theta_{\text {nat }} \gg \theta_{n}$ where $n=1$ and the damping factors for the cavity fluid and transducer are small, (i.e., $\zeta_{n} \ll<1, n=0,1,2,3, \ldots N$, and $\left.\zeta \ll 1\right)$. Recall that $\alpha$ is the ratio of fluid mass in the cavity to transducer piston mass located at $\xi=1$ (or $x=\ell$ ), and that $\theta_{1}$ is the first non-dimensional natural frequency of an open-closed cavity.

\section{Pressure Ratio}

The maximum normalized pressure (or non-dimensional pressure ratio) on the plots is defined as:

$$
\text { pressure ratio }=\frac{\left|\begin{array}{l}
P(\tau) \\
\text { ouppu }
\end{array}\right|}{\left|\begin{array}{l}
\max \\
P_{\text {inpux }}(\tau)
\end{array}\right|}
$$

where

$$
\left|P_{\text {ouppux }}(\tau)\right|_{\max }=\left|\frac{1}{\rho_{o} c_{o}^{2}}\left[p(1, \tau)-p_{o}\right]\right|_{\max }
$$

and

$$
\left|P_{i \text { inpur }}(\tau)\right|=\left|\frac{1}{\rho_{o} c_{o}^{2}}\left[\underset{\text { nor }}{p}(0, \tau)-p_{o}\right]\right|_{\max }=1.0
$$

where $\mid \begin{aligned} & P_{\text {inpus }}(\tau) \\ & \left.\right|_{\max }\end{aligned}$ is the maximum normalized non-dimensional input pressure and equals unity. The specified but normalized input pressure $\underset{\text { nor }}{p}(0, \tau)$ is expressed by Eq. (58)

$$
\underset{n o r}{p}(0, \tau)=\rho_{o} c_{o}^{2}\left[\frac{p_{o}}{\rho_{o} c_{o}^{2}}+\frac{p\left(0, \frac{c_{o}}{\ell} t\right)}{\left|p\left(0, \frac{c_{o}}{\ell} t\right)\right|_{\max }}\right]
$$


where $p\left(0, \frac{c_{o}}{\ell} t\right)$ is determined from the specified input pressure $p(0, t)$ by multiplying each time point $t_{i}$ by $\frac{c_{0}}{\ell}$. Note that the maximum absolute value of the last term within the brackets in Eq. (58) is unity. That is,

$$
\left|\frac{|p(0, t)|}{|p(0, t)|_{\max }}\right|_{\max }=\left|\frac{p\left(0, \frac{c_{o}}{\ell} t\right)}{\left|p\left(0, \frac{c_{o}}{\ell} t\right)\right|_{\max }}\right|_{\max }=1.0
$$

Recall that the specified input pressure $p(0, t)$ acts on the massless piston located at $\xi=0$ (or $x=0$ ) and that $p(0, t)$ is specified with respect to the real time $t$. Note that $\tau=\frac{c_{o}}{\ell} t$. Also note that for the pressure wave to travel the length $\ell$ of the cavity at a speed of $c_{o}$ requires a non-dimensional time of unity; i.e., $\tau=1$. Substitution of Eq. (58) into Eq. (57) and utilizing Eq. (59) satisfies Eq. (57).

\section{Time Ratio}

The time ratio on the plots is defined as: 8

$$
\text { time ratio }=\frac{\tau_{1}}{T_{1}}
$$

$\tau_{1}$ is shown on each "forcing function" plot and is associated with the normalized nondimensional specified input pressure $\underset{\text { input }}{P(\tau)}$ and with the specified normalized input pressure $\underset{\text { nor }}{p}(0, \tau) . \tau_{1}$ is a non-dimensional time (i.e., $\tau_{1}=\frac{c_{o}}{\ell} t_{1}$ ) and is either a rise time, a pulse duration, or the half-period of a sinusoid. Specifically, $\tau_{1}$ is the rise time of a ramp-up input pressure; the time duration of a step input pressure; the time duration of an isosceles triangular input pressure; the time for a half-period of sinusoidal input pressure. The maximum amplitude of the normalized non-dimensional specified input pressure $\left.P_{\text {input }}(\tau)\right|_{\max }$ is unity for all "forcing functions." $T_{1}$ is the non-dimensional first natural period of a cavity open on one end and closed on the other

$8 T_{1}$ is used in the time ratio to normalize the ratio. 
end. For example, the $\mathrm{n}^{\text {th }}$ non-dimensional natural frequency for an open-closed cavity is given by Eq. (60). Refer to Eq. (46).

$$
\theta_{n}=(2 n-1) \frac{\pi}{2} \quad n=1,2,3, \ldots . . N
$$

The $\mathrm{n}^{\text {th }}$ non-dimensional natural period is given by Eq. (61).

$$
T_{n}=\frac{2 \pi}{\theta_{n}} \quad n=1,2,3, \ldots . N
$$

or

$$
T_{n}=\frac{4}{(2 n-1)} \quad n=1,2,3, \ldots . . N
$$

Therefore, $T_{1}=4$. Recall that $\theta_{n}=\omega_{n} \frac{\ell}{c_{o}}$ and that $\omega_{n}=(2 n-1) \frac{\pi}{2} \frac{c_{o}}{\ell}$ for an open-closed cavity.

\section{Plot Parameters}

The non-dimensional physical system parameters, $\theta_{\text {nat }}, \zeta_{n}, \zeta$, and $\alpha$, normalized nondimensional specified input pressure $P(\tau)$, cavity configuration and boundary conditions associated with the response plots are provided with each plot. The general boundary condition implies that the solution vector to Eq. (41) is utilized; the simplified boundary condition implies that the solution vector to Eq. (46) is utilized. The maximum number of terms $\mathrm{N}$ used in the series solution for $\bar{w}_{c}(n, \tau)$ [for example, in Eqs. (41) and (46)] varied from 12 to 18 to satisfy a convergence criteria as follows. During the process of numerically integrating Eq. (41) or Eq. (46) for a particular value of $\tau_{1}$ which is associated with the normalized non-dimensional specified input pressure $P(\tau)$ or "forcing function," the maximum amplitude of each Fourier transform harmonic for $n=0,1,2,3, \ldots N$ was computed and normalized to a maximum value of one for all $n$. If the average of this quantity for the last two values of $n(\mathrm{~N}$ and $\mathrm{N}-1)$ did not exceed one percent, the numerical solution for that particular value of $\tau_{1}$ was considered to have converged. This provided excellent convergence for the solution within the parameter space investigated. 
On some of the response spectra plots for sinusoidal inputs, the response function may appear double valued for small values of the time ratio $\frac{\tau_{1}}{T_{1}}$. This results from using a "smoothing function" for a small set of time ratio data points in the region where $0<\frac{\tau_{1}}{T_{1}}<0.5$. For linear systems the response spectra plots are single valued for each time ratio point $\frac{\tau_{1}}{T_{1}}$.

On the response spectra plots denoted as a generalized boundary condition, the nondimensional first natural frequency of the transducer is equal to unity (i.e., $\theta_{\text {nat }}=1$ ) on the first set of plots, equal to $\pi$ on the second set of plots, and equal to $\pi^{2}$ on the third set of plots.

\section{Normalized Non-Dimensional Specified Input Pressures}

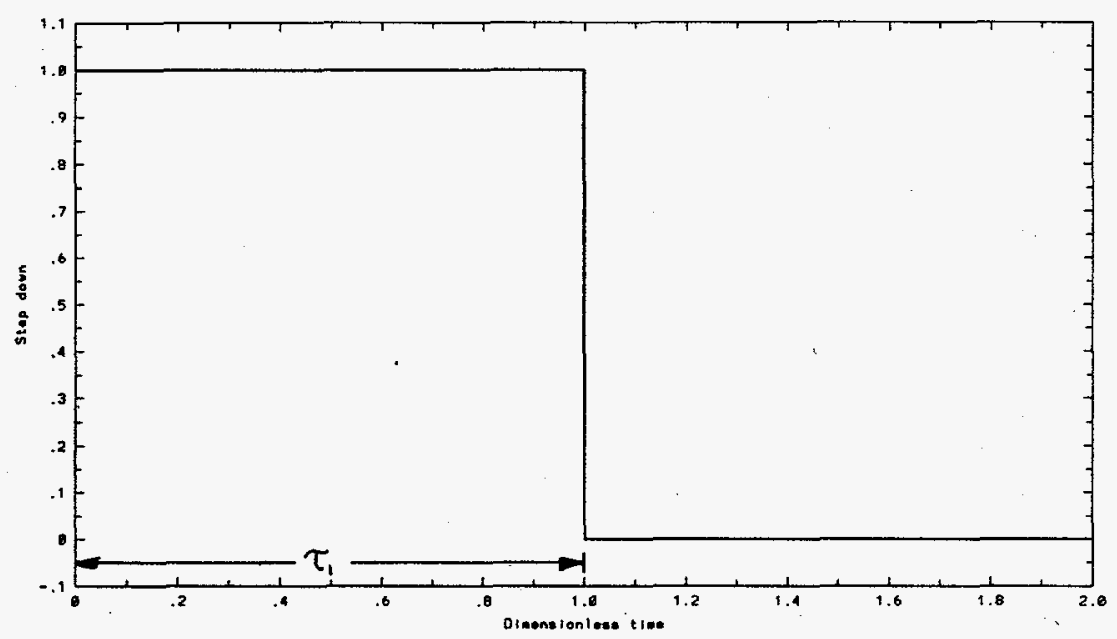

Figure 3

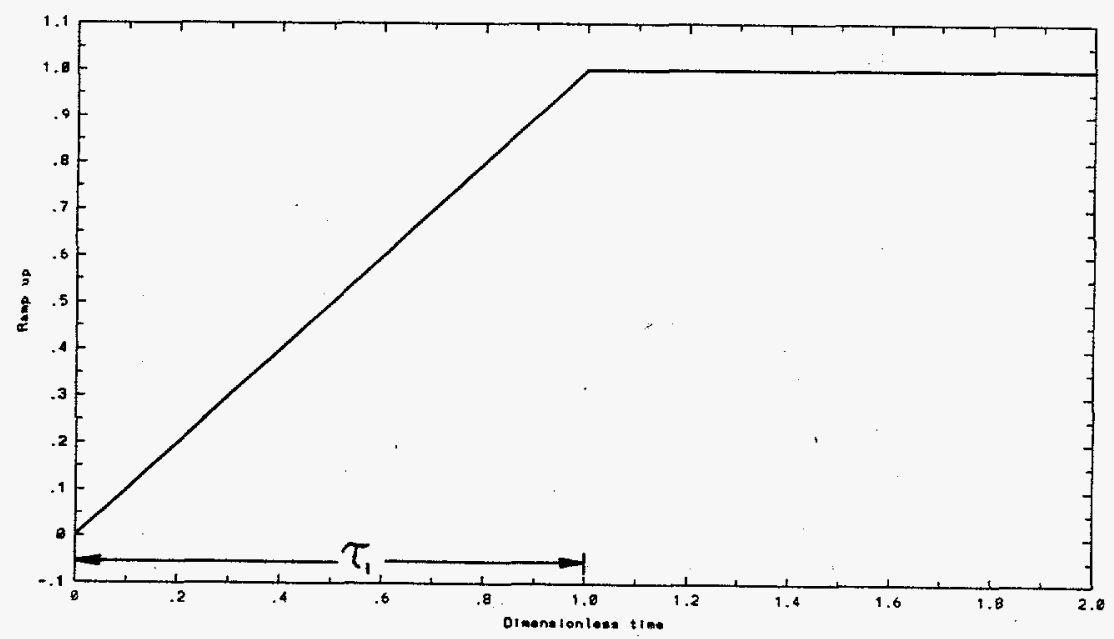

Figure 4 


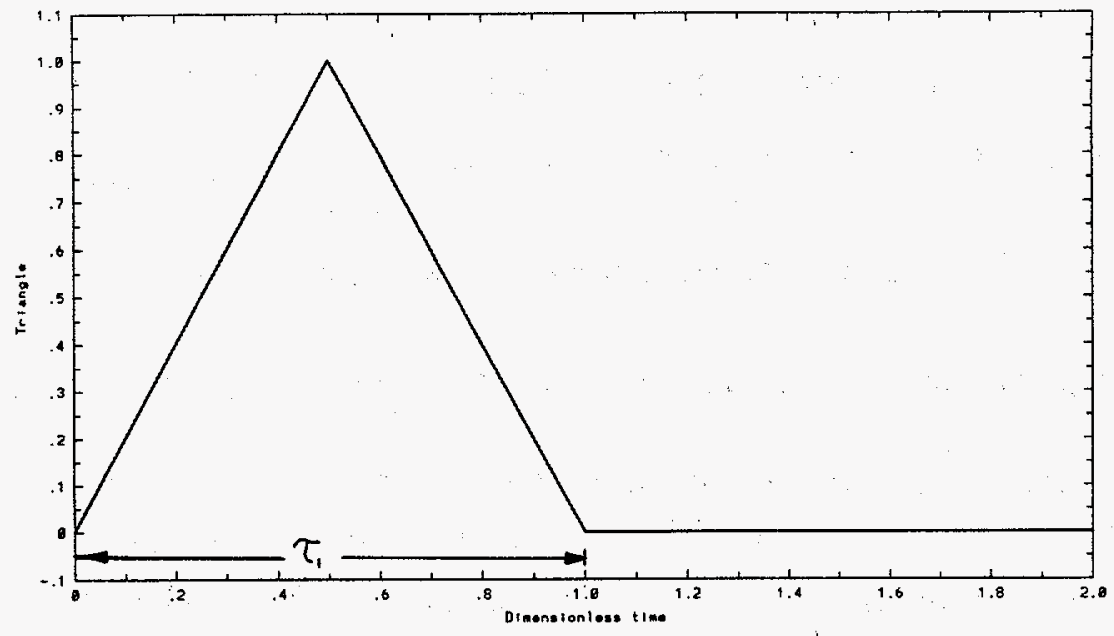

Figure 5

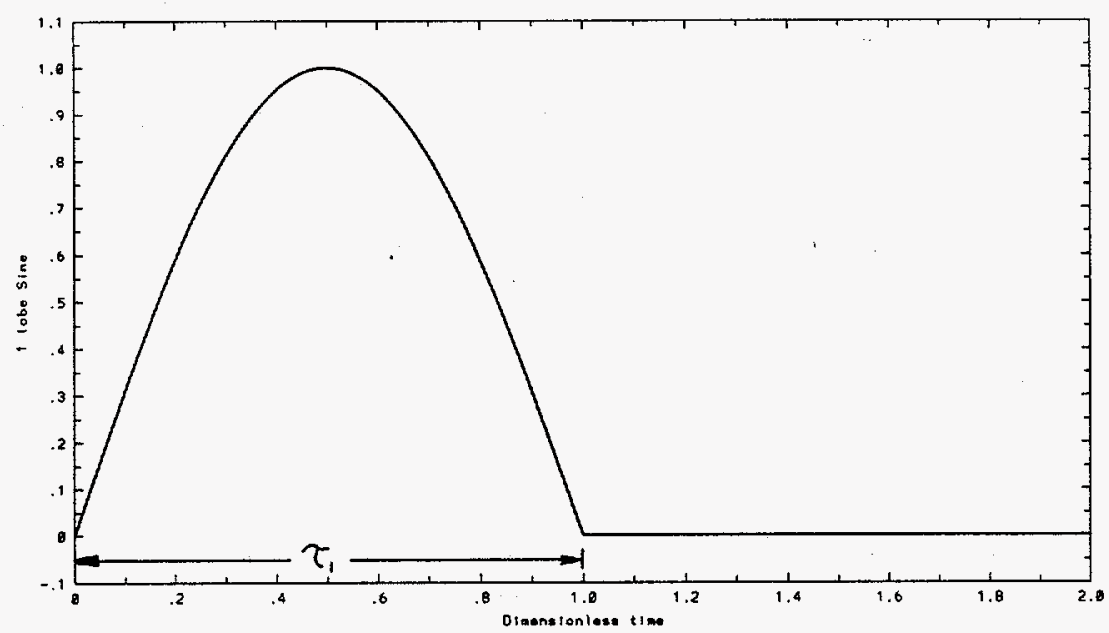

Figure 6

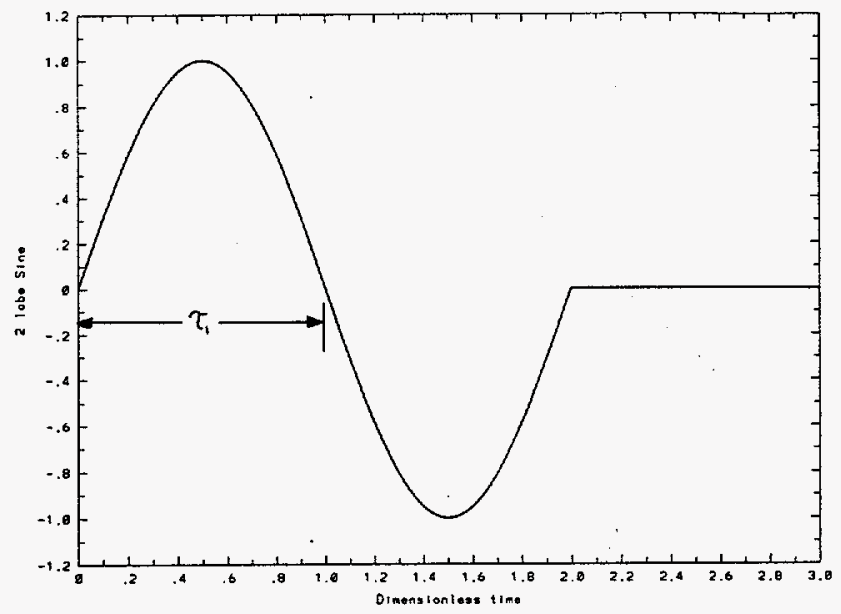

Figure 7 


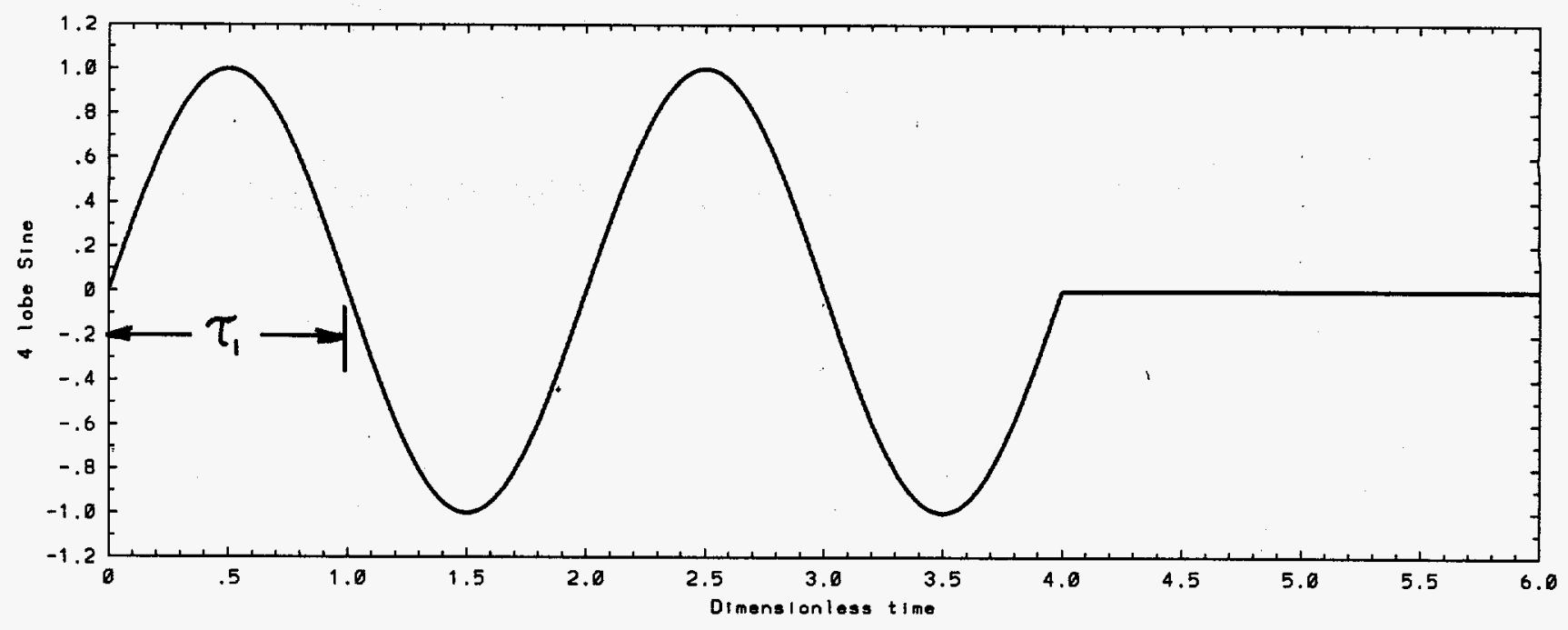

Figure 8

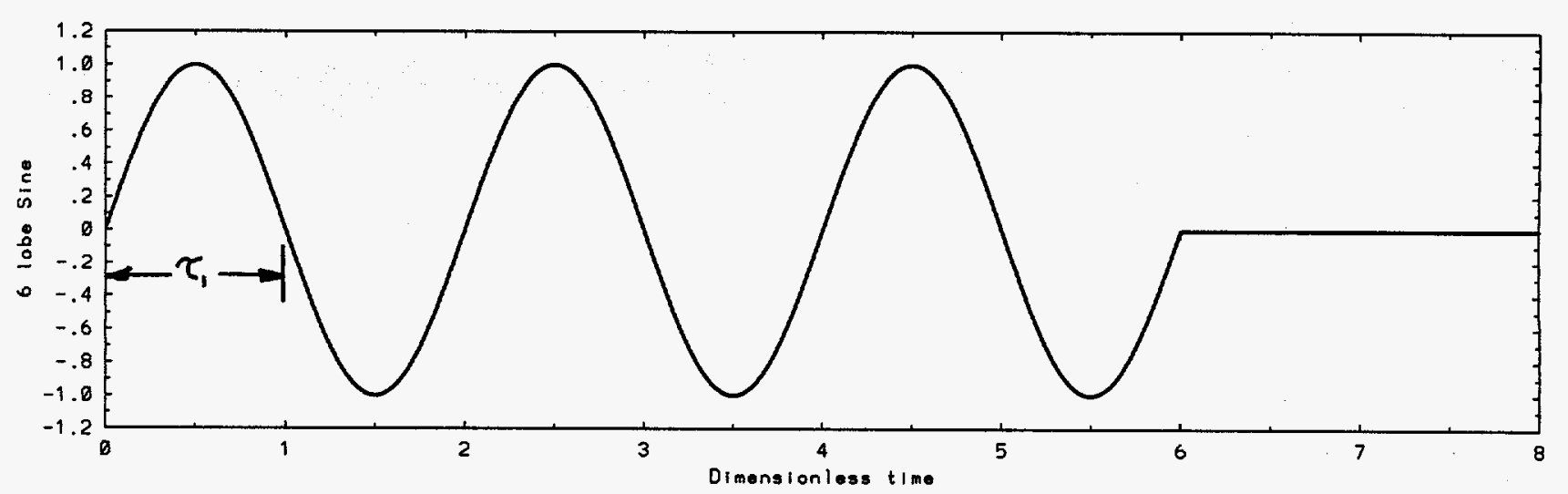

Figure 9

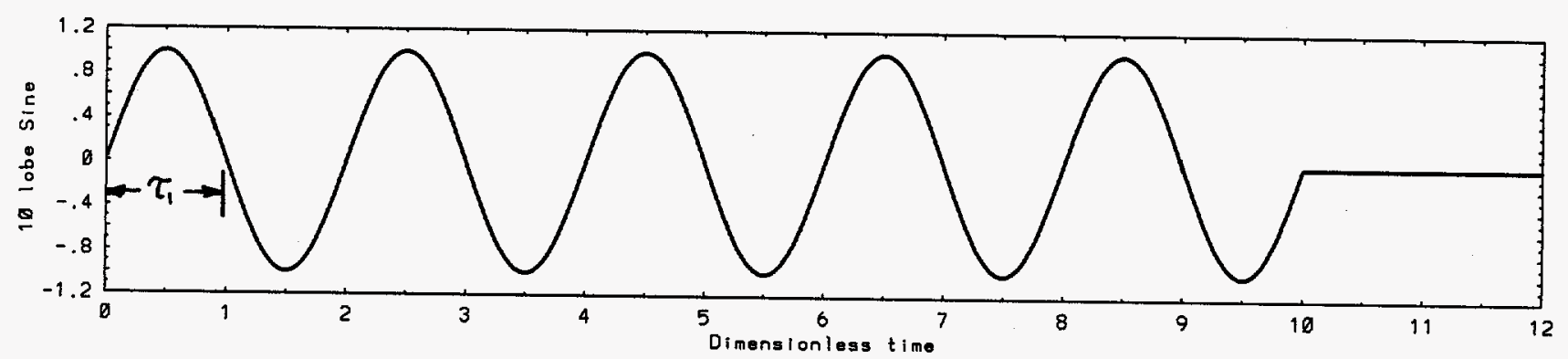

Figure 10 



\section{Pressure Response Spectra}

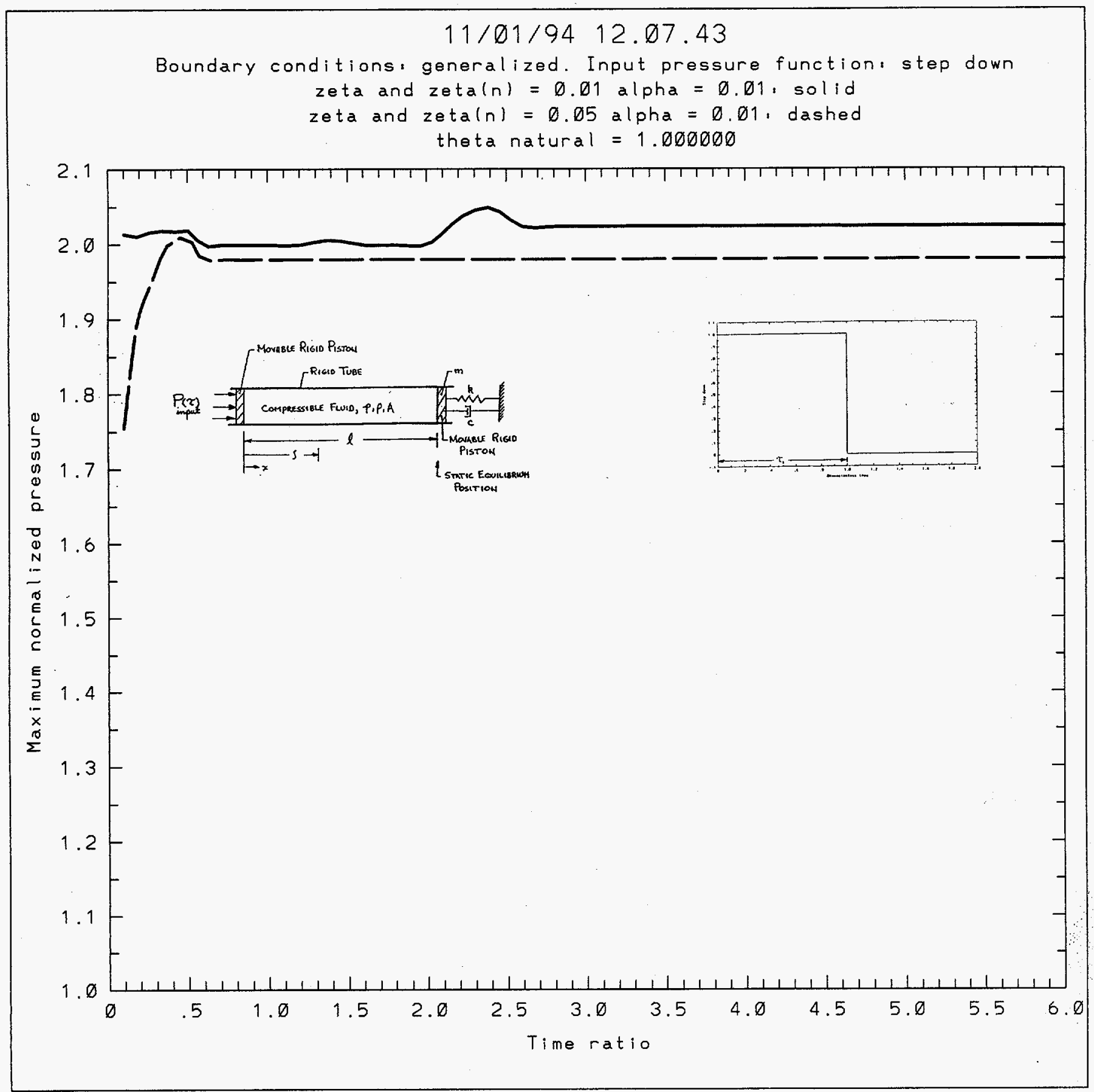

Figure 11 


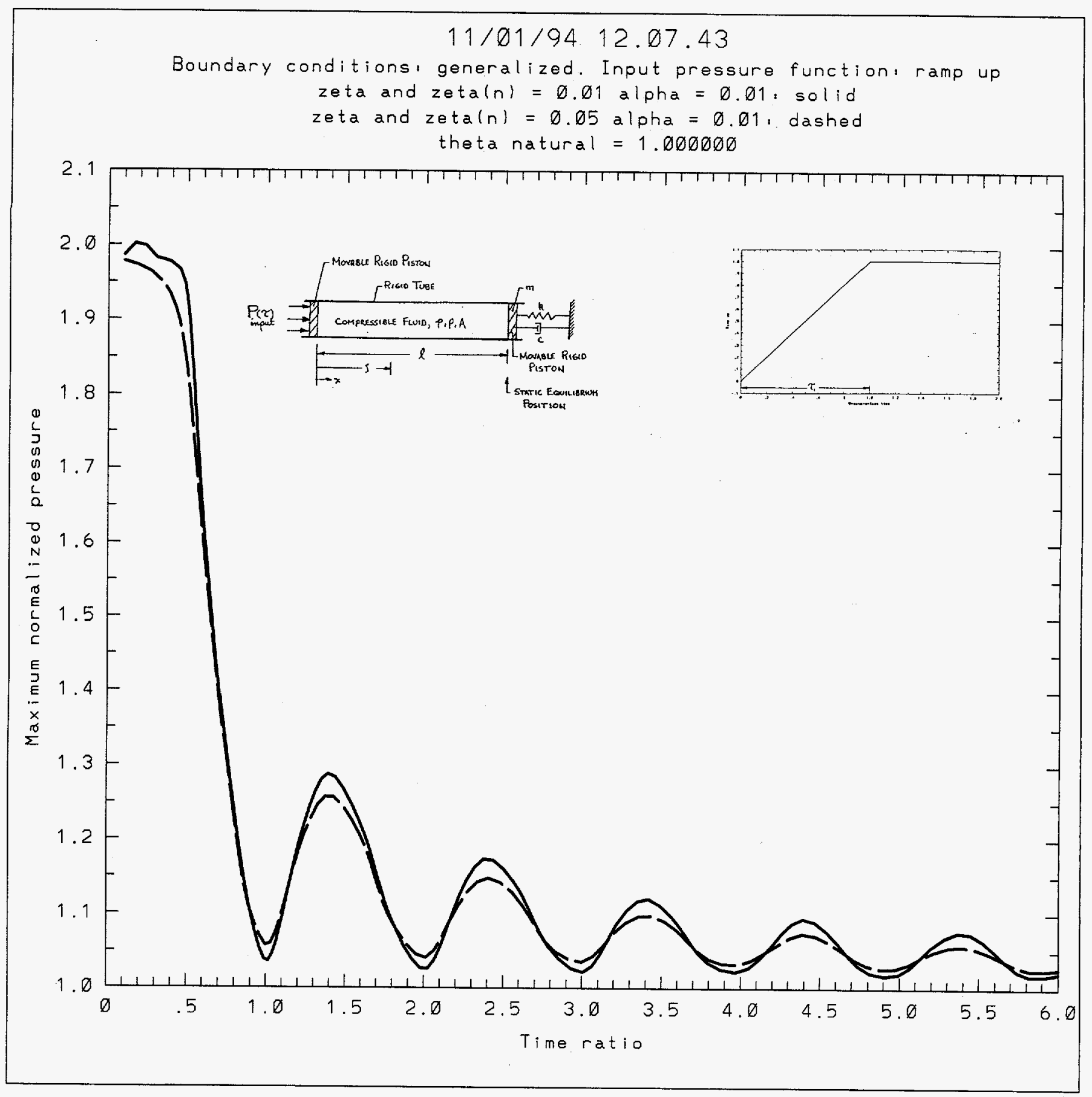

Figure 12 


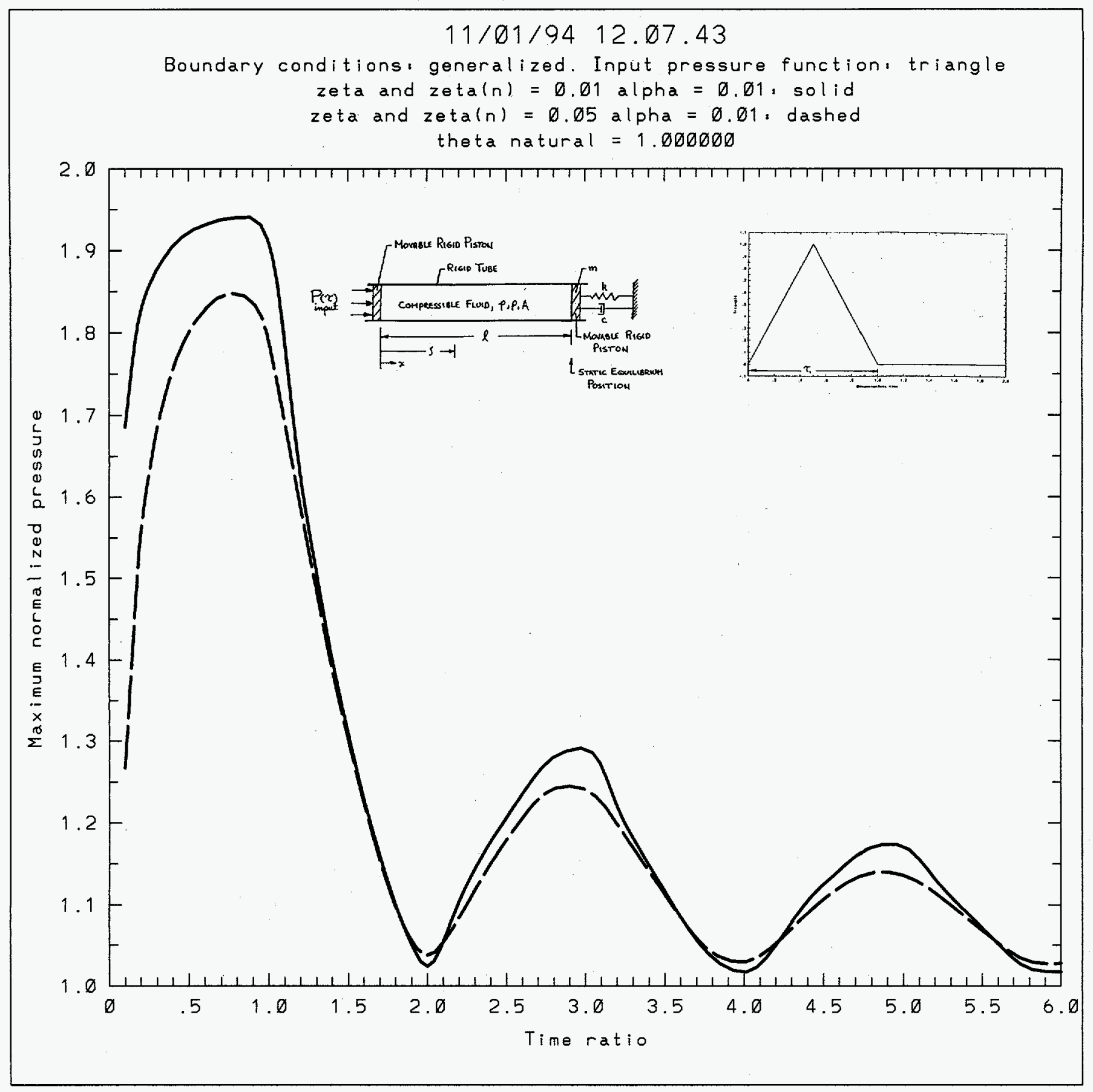

Figure 13 


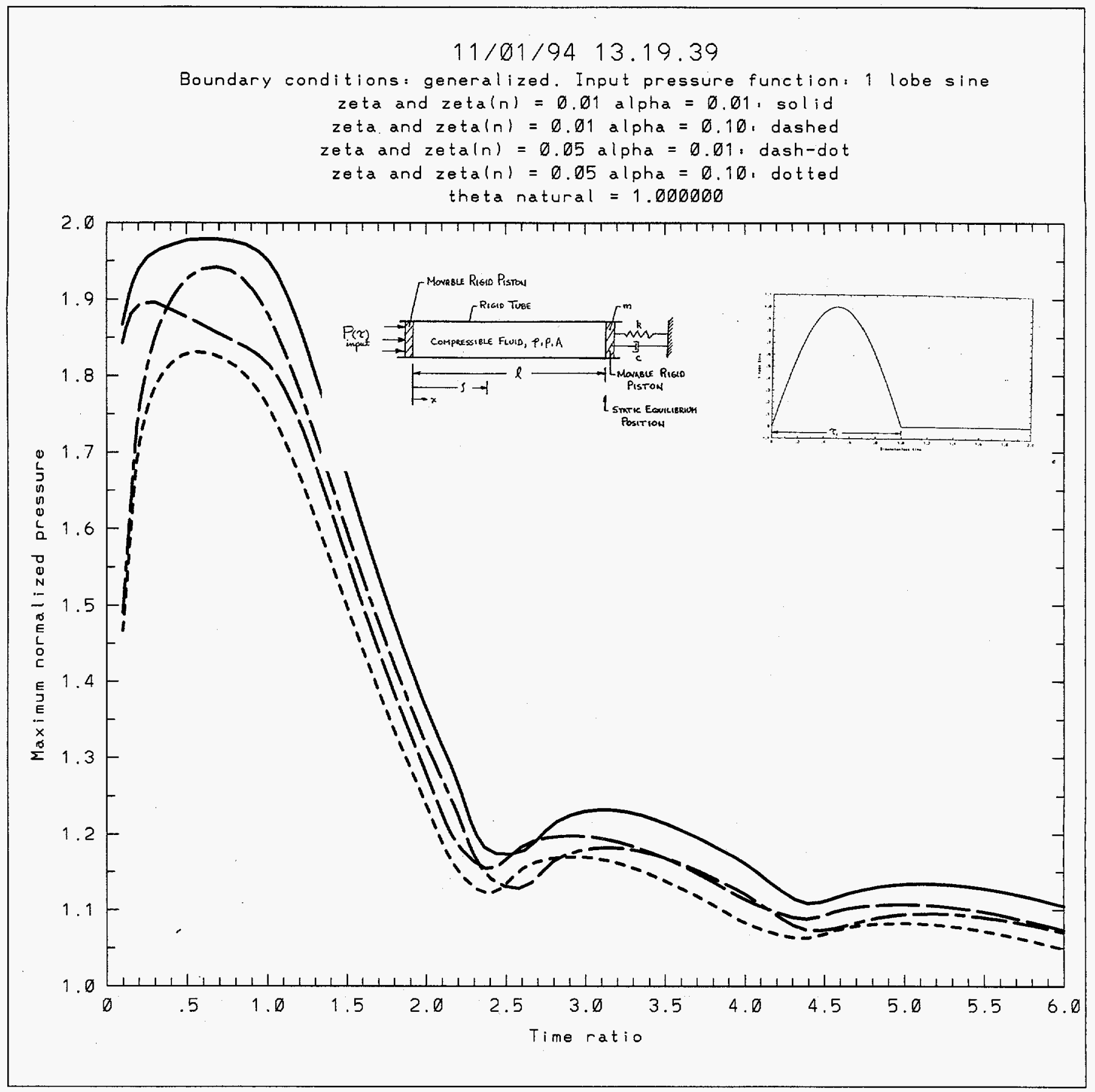

Figure 14 


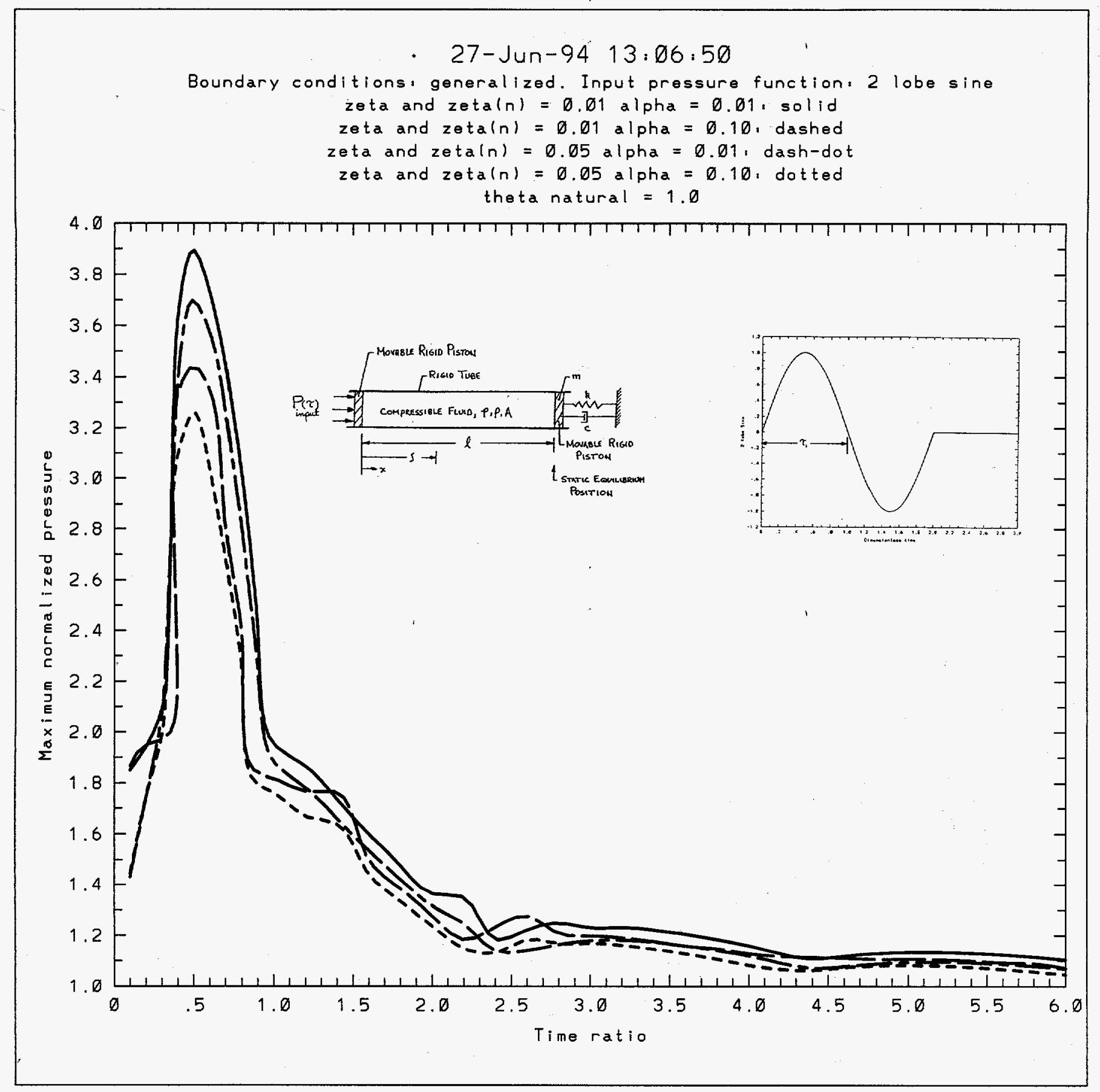

Figure 15 


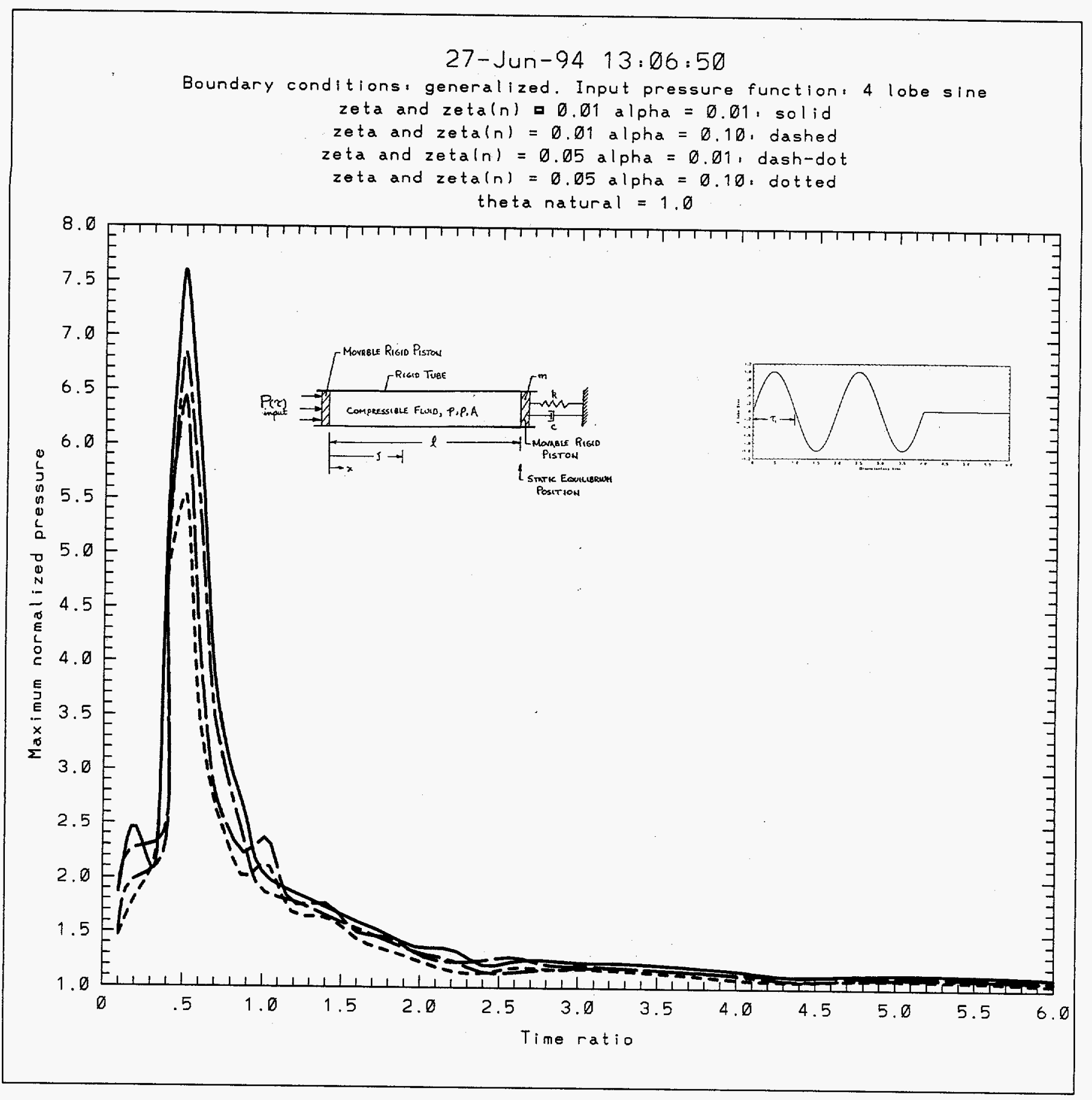

Figure 16 


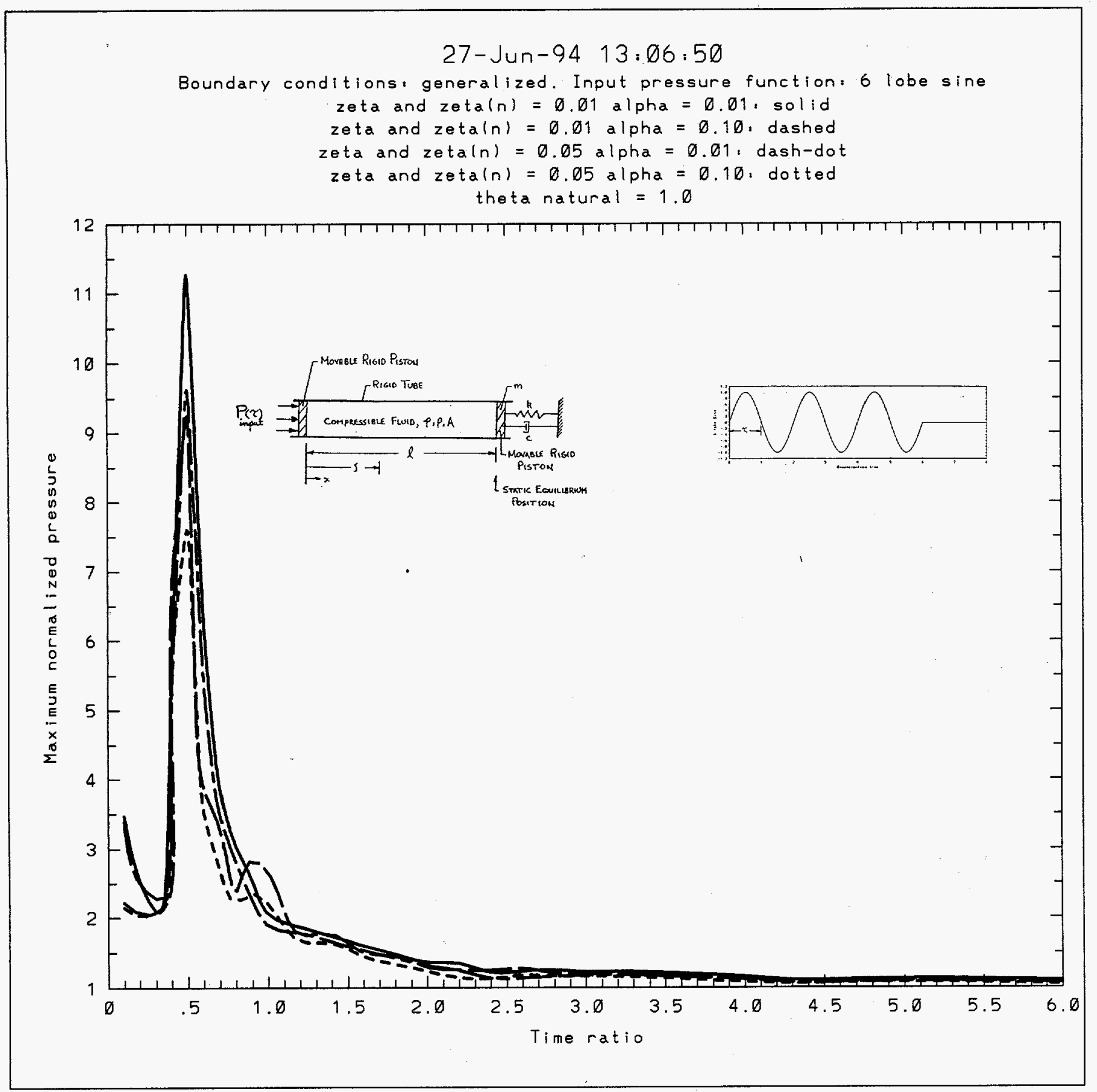

Figure 17 


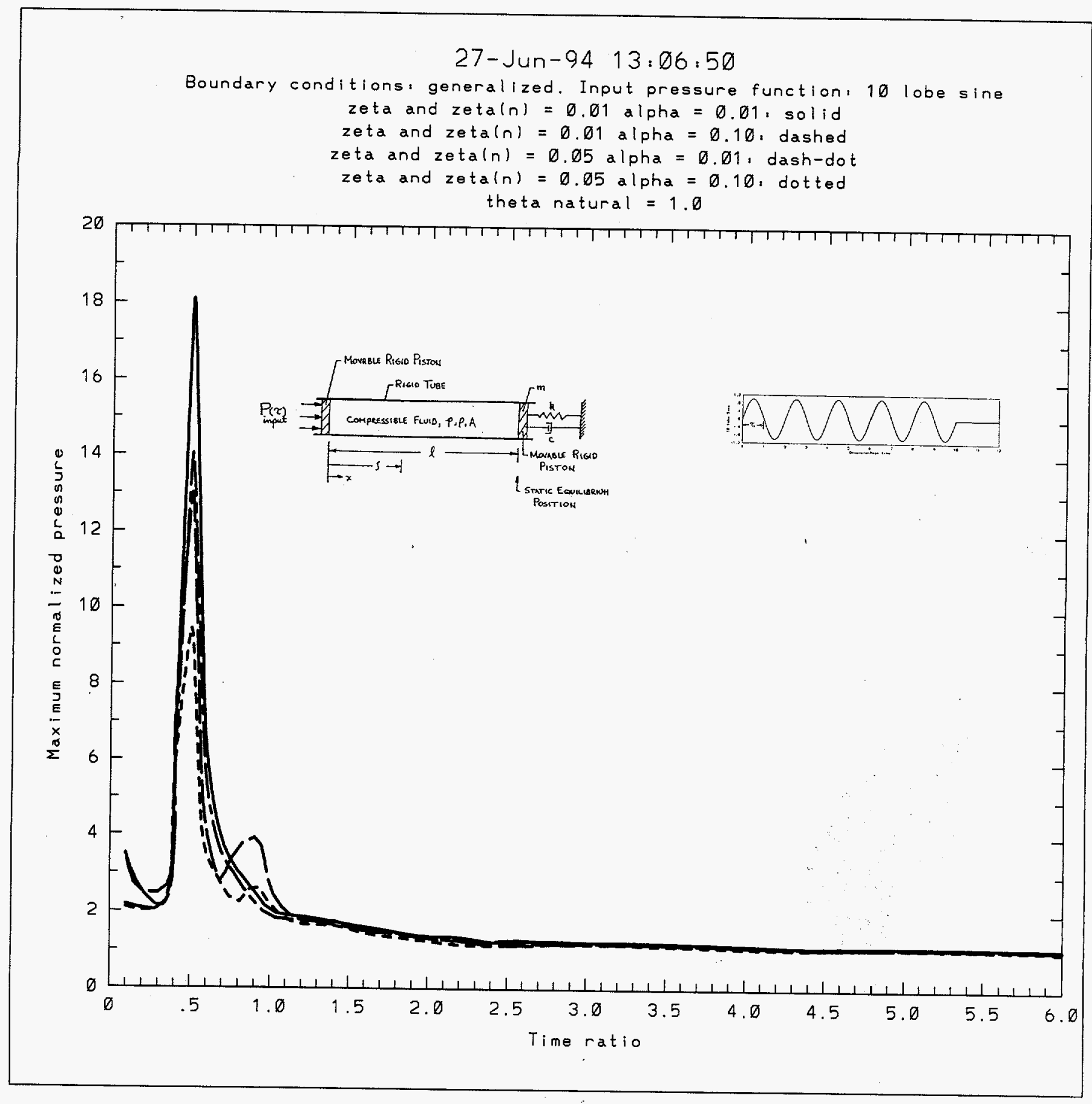

Figure 18 


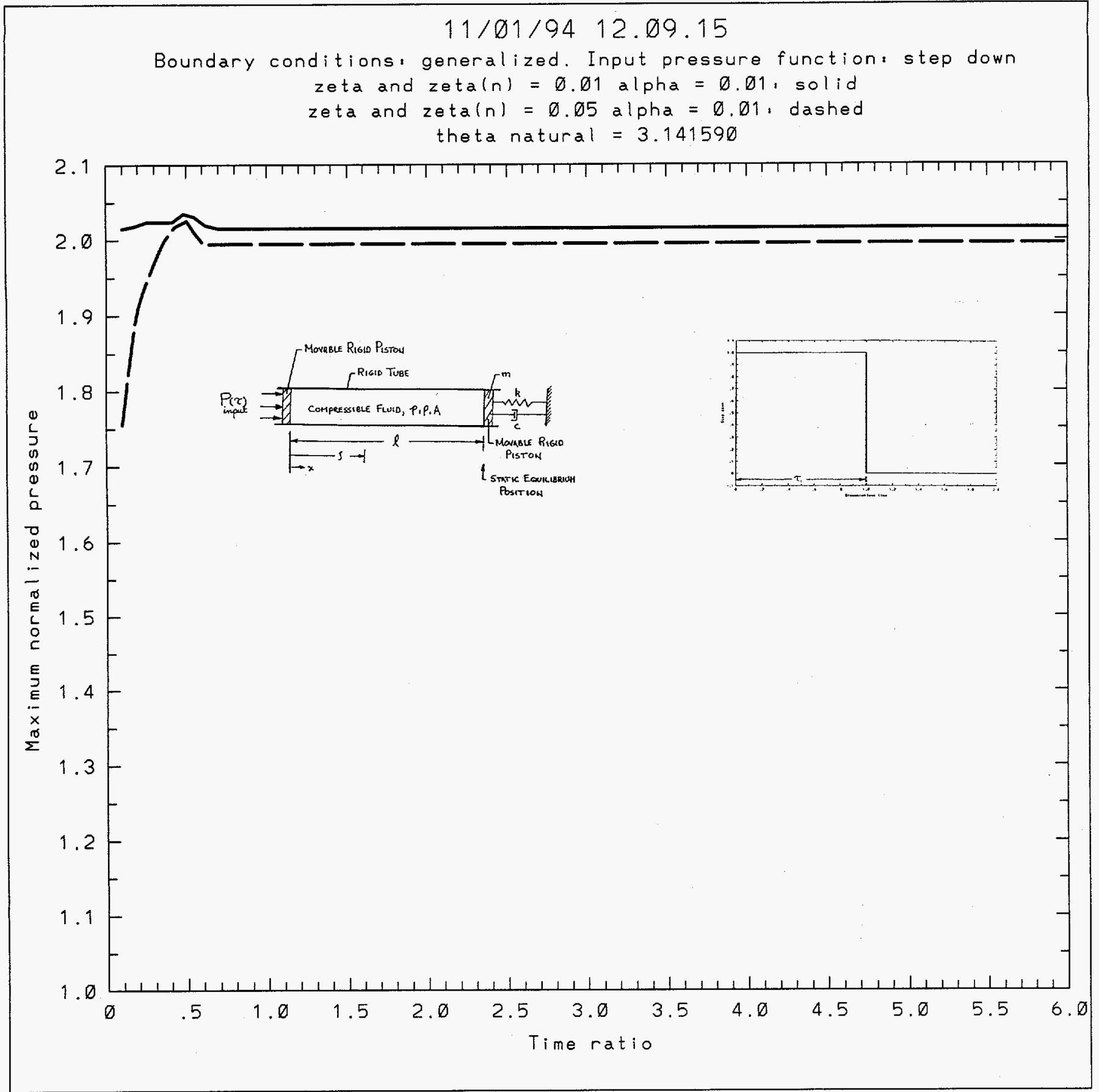

Figure 19 


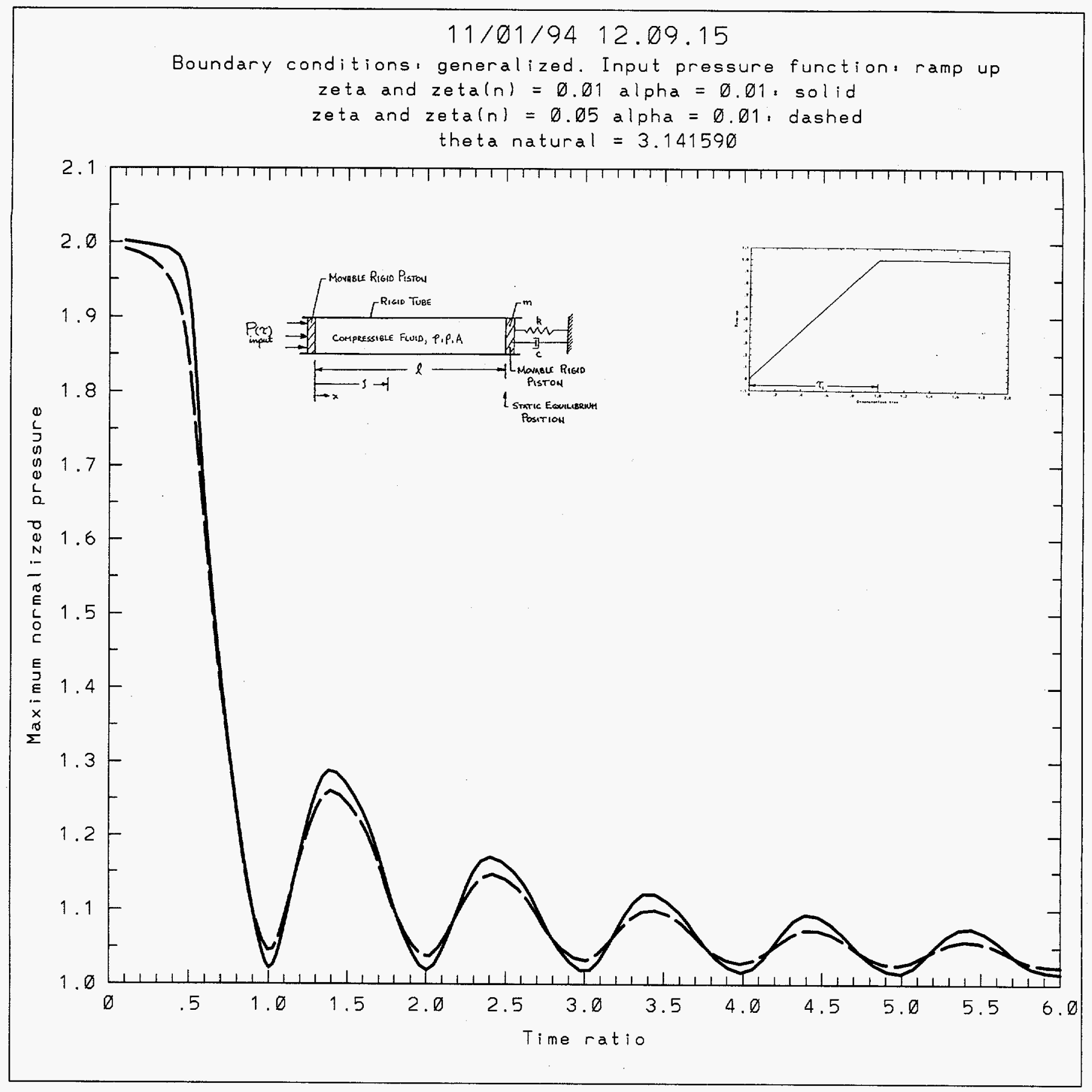

Figure 20 


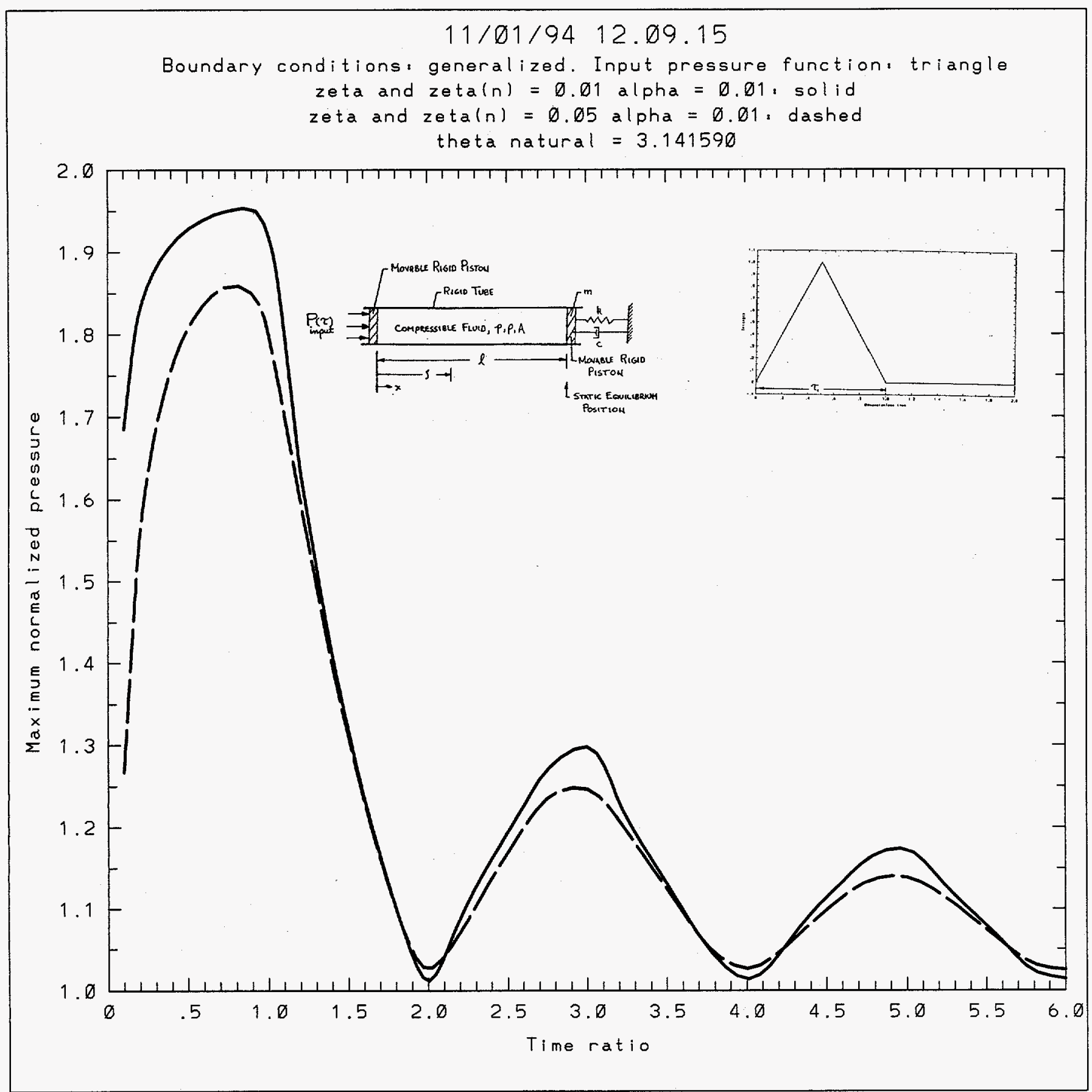

Figure 21 


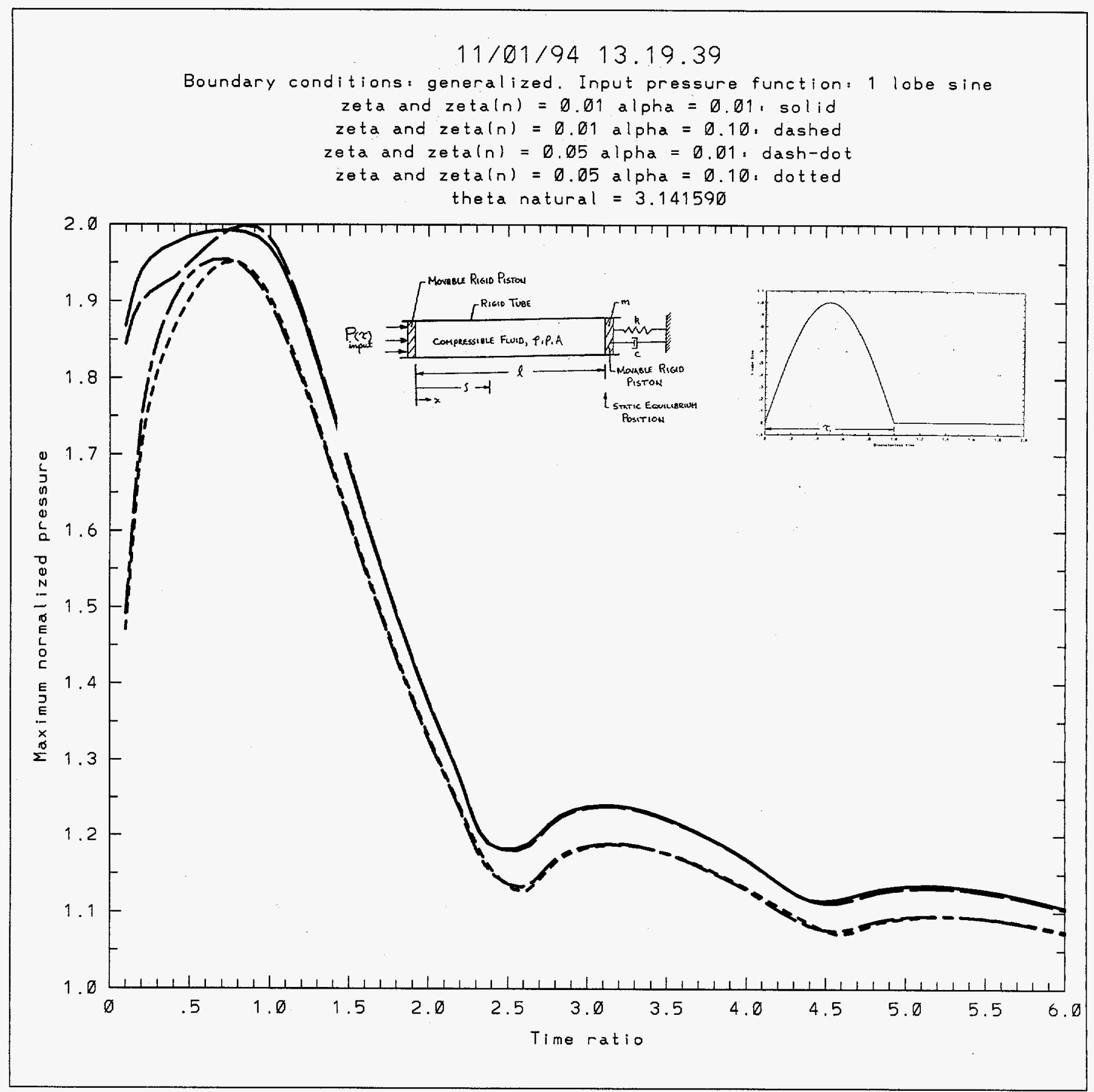

Figure 22 


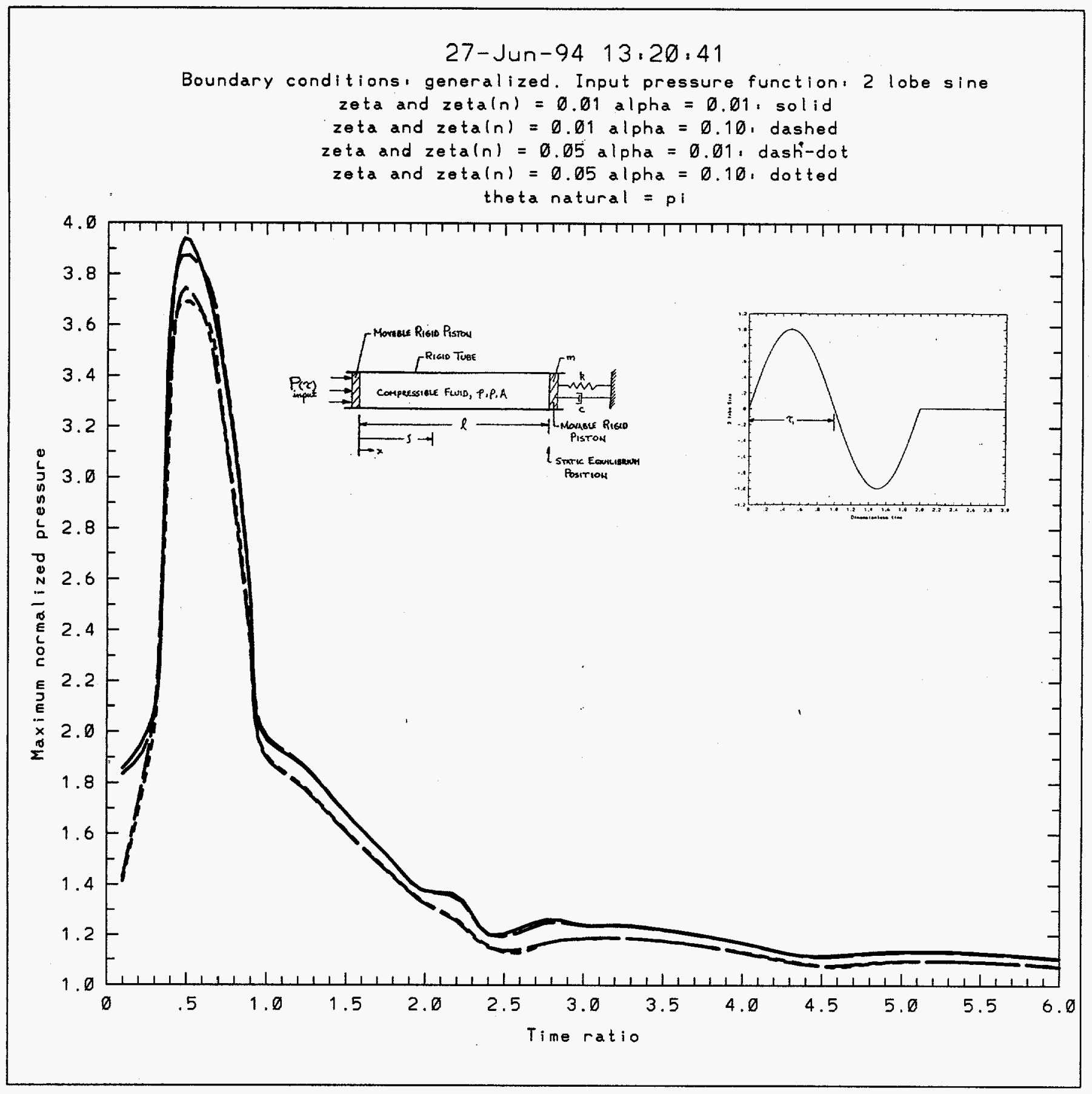

Figure 23 


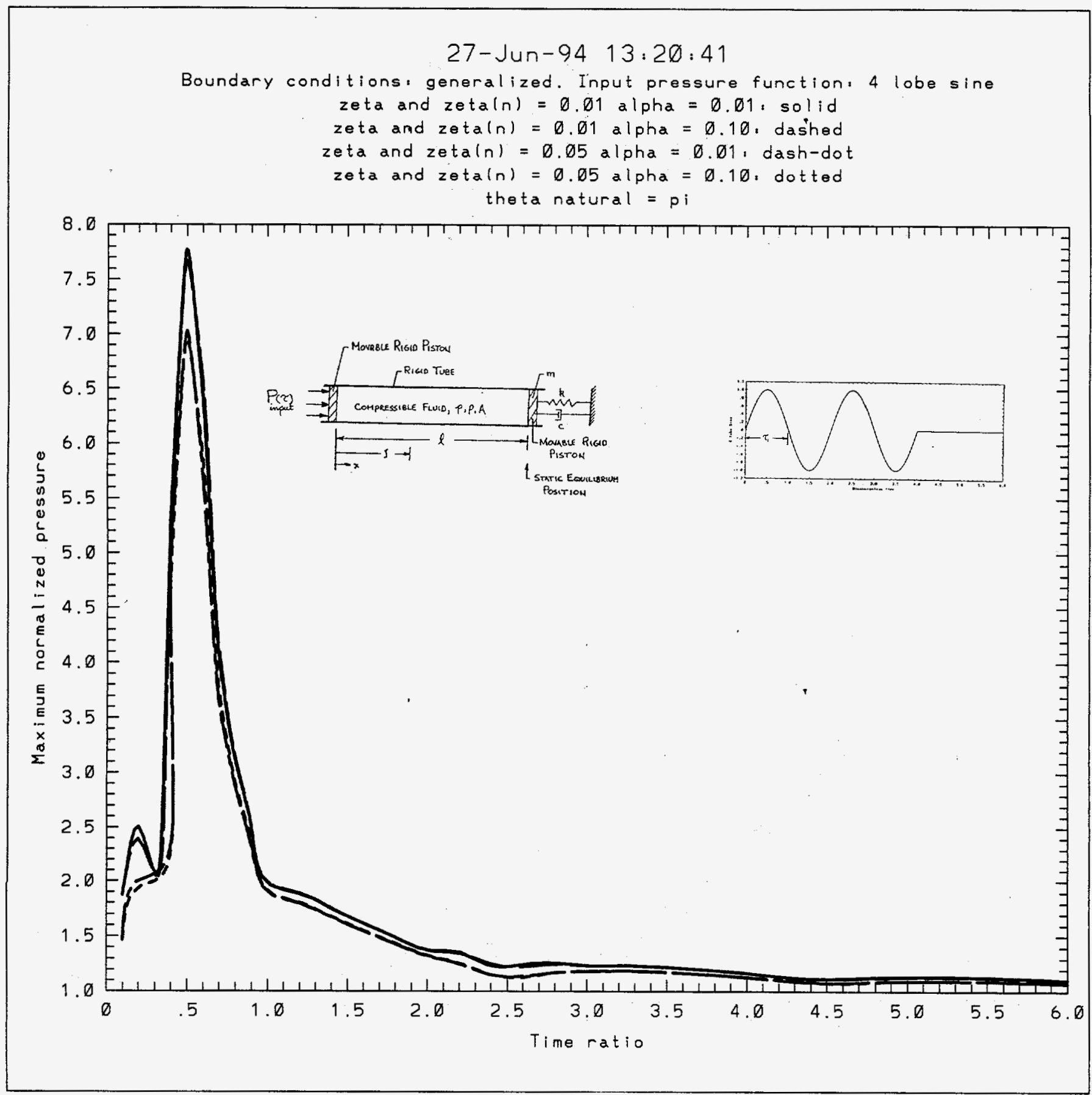

Figure 24 


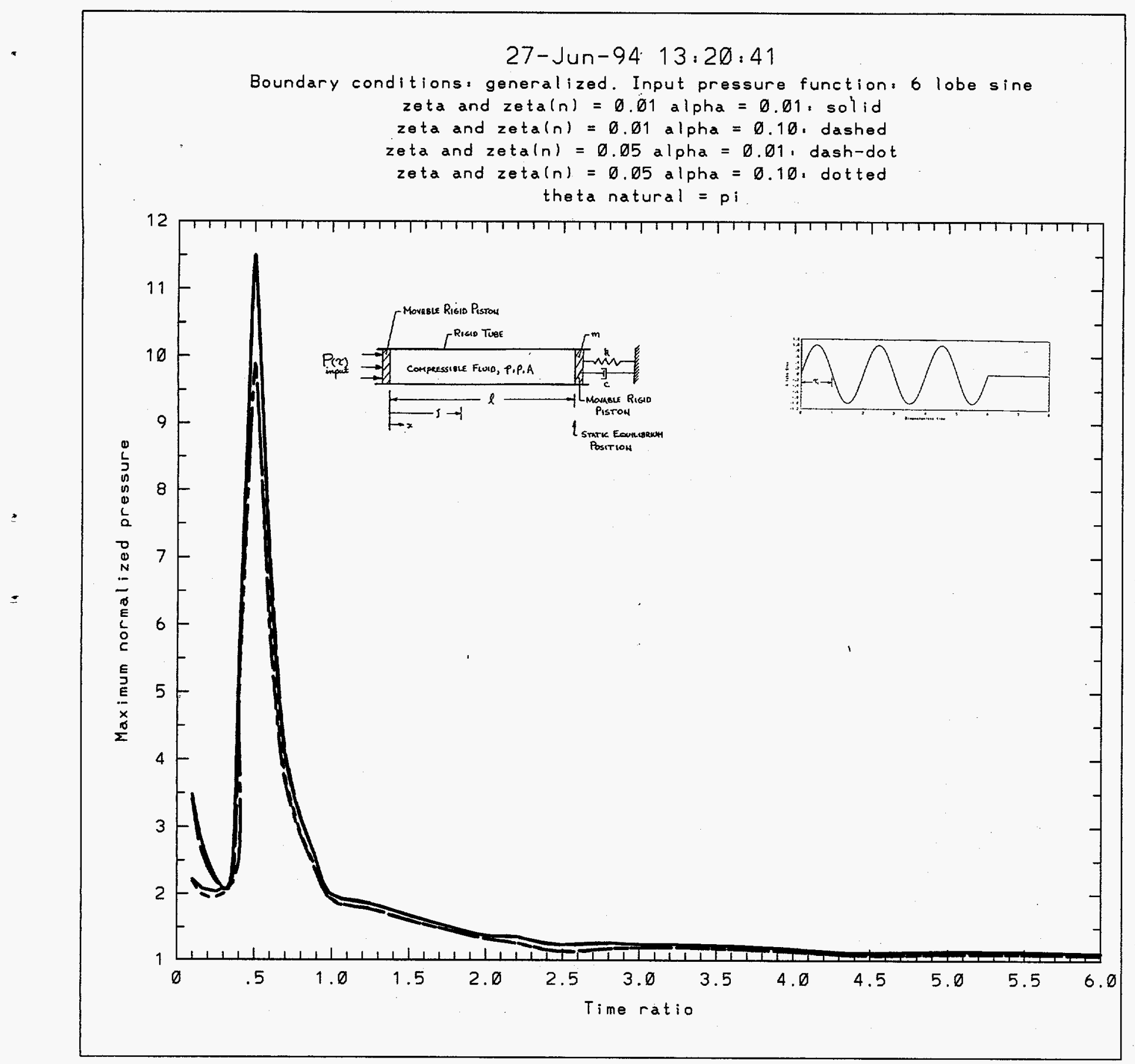

Figure 25 


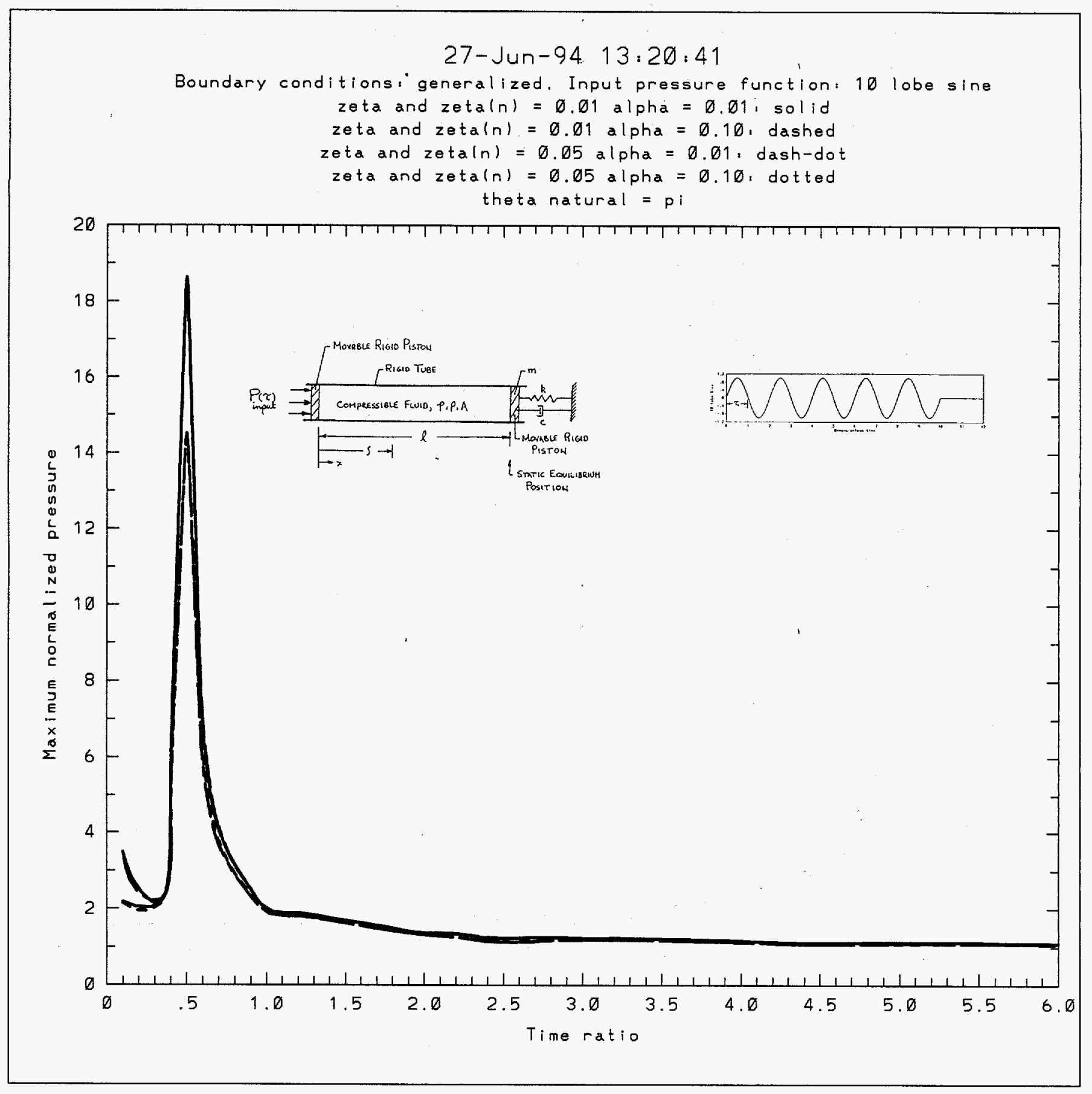

Figure 26 


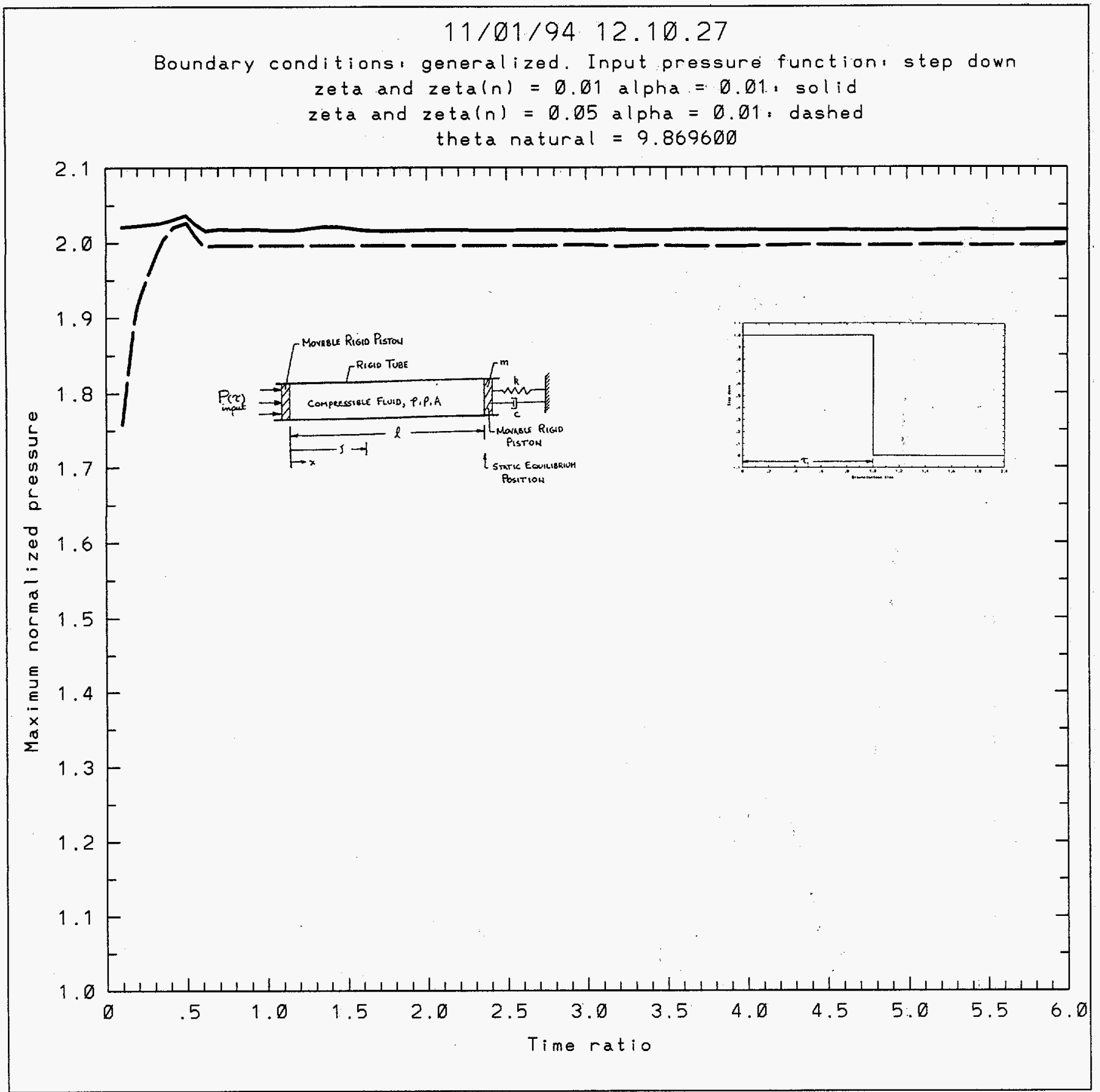

Figure 27 


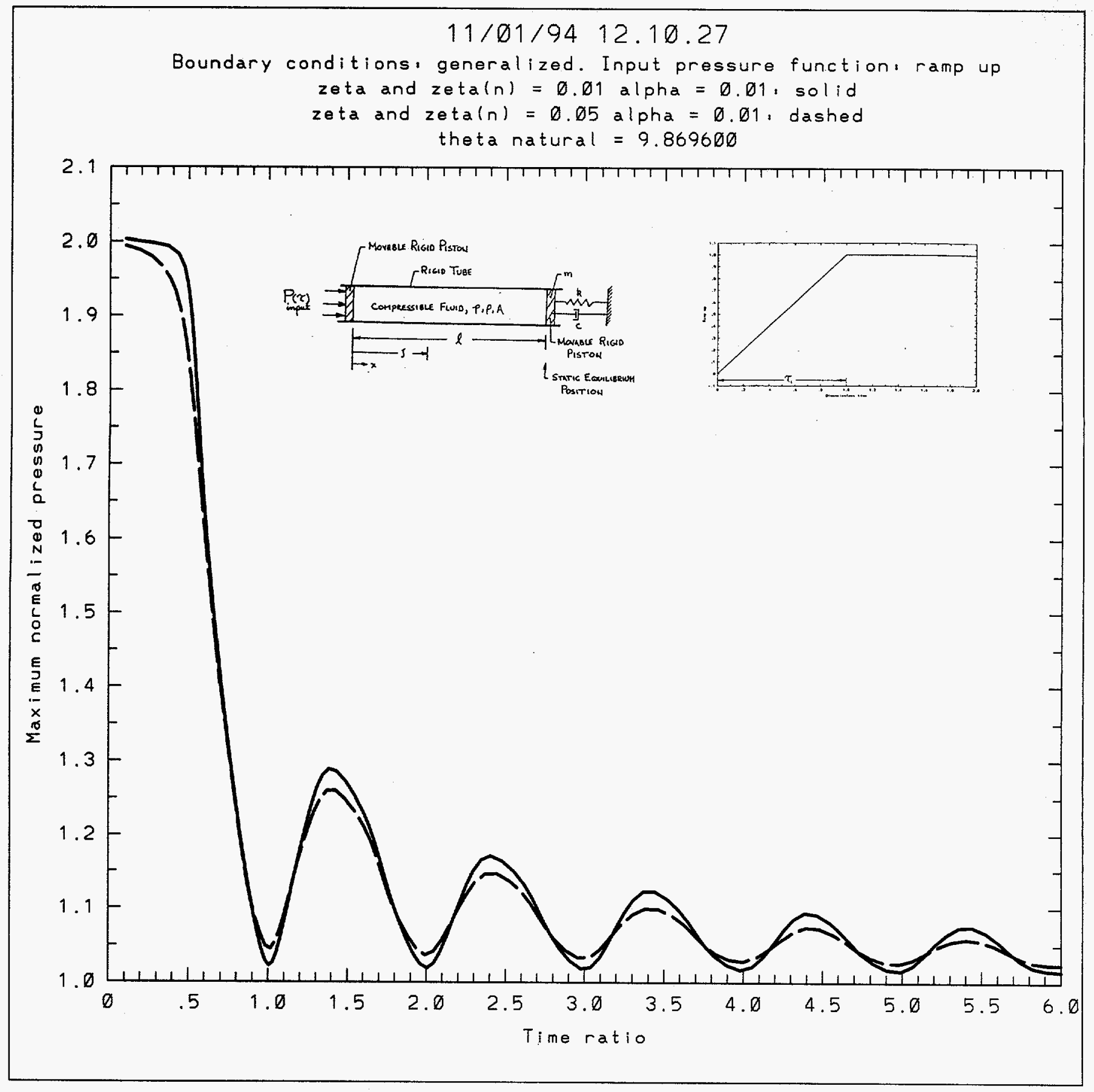

Figure 28 


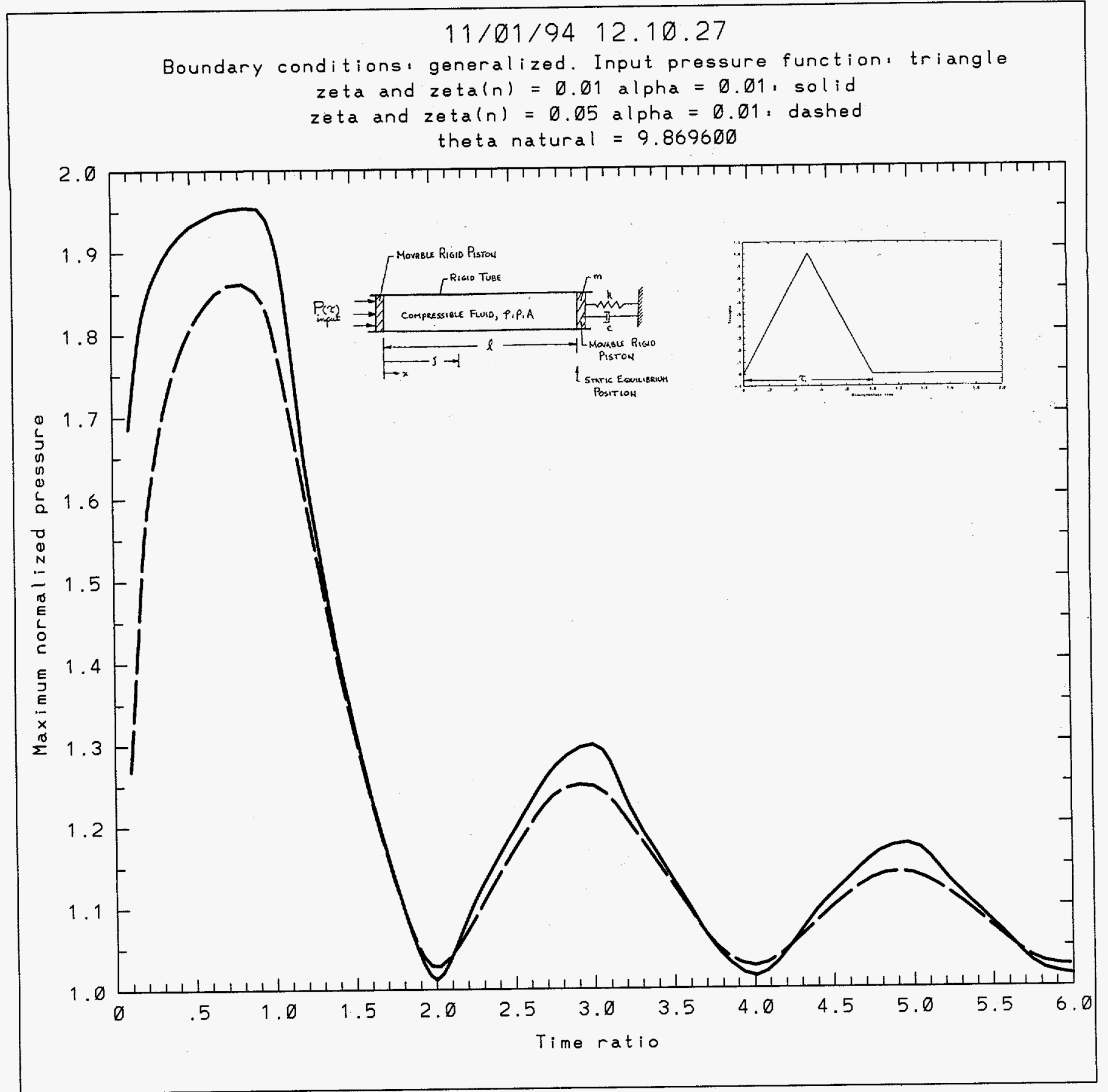

Figure 29 


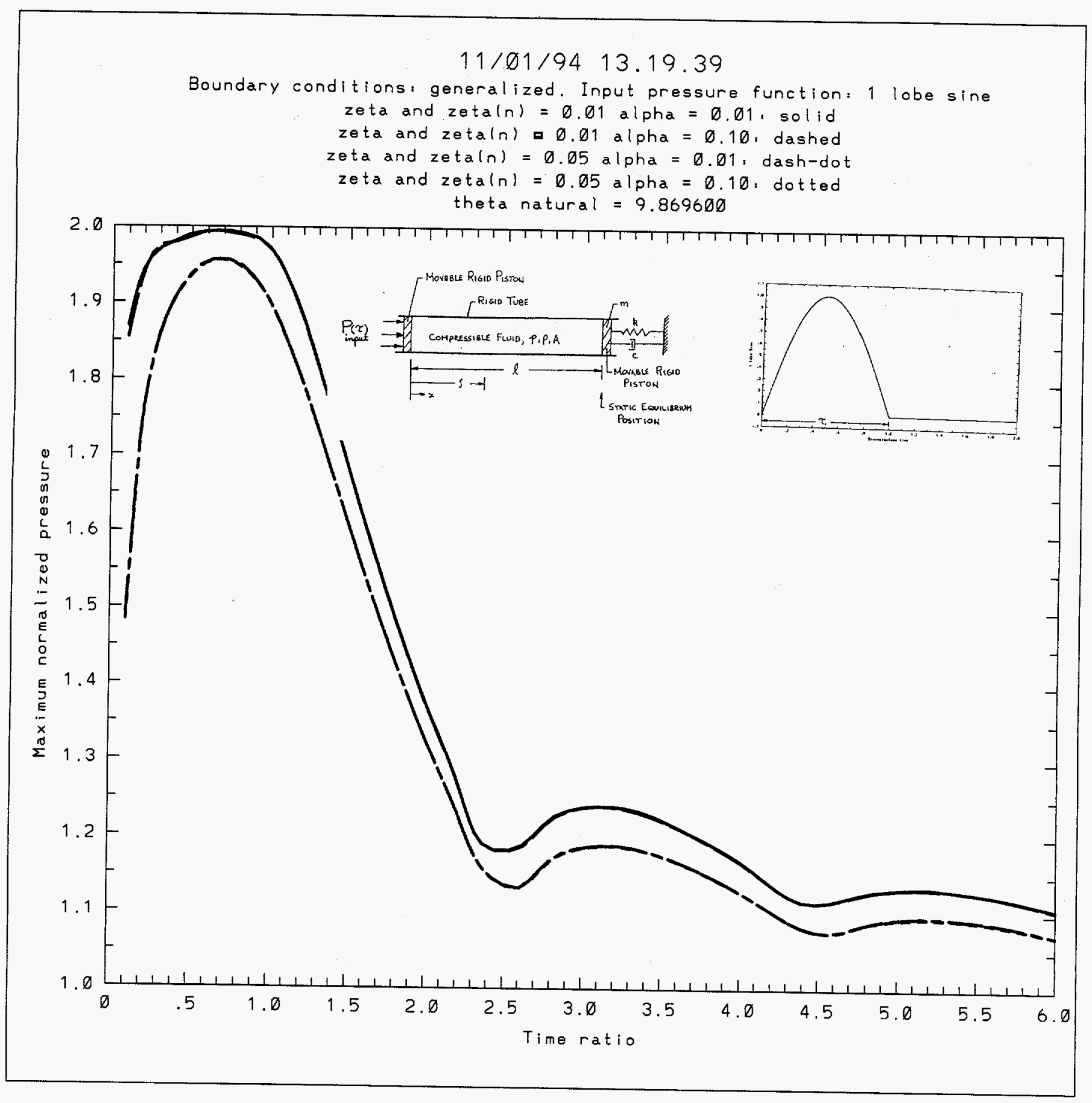

Figure 30 


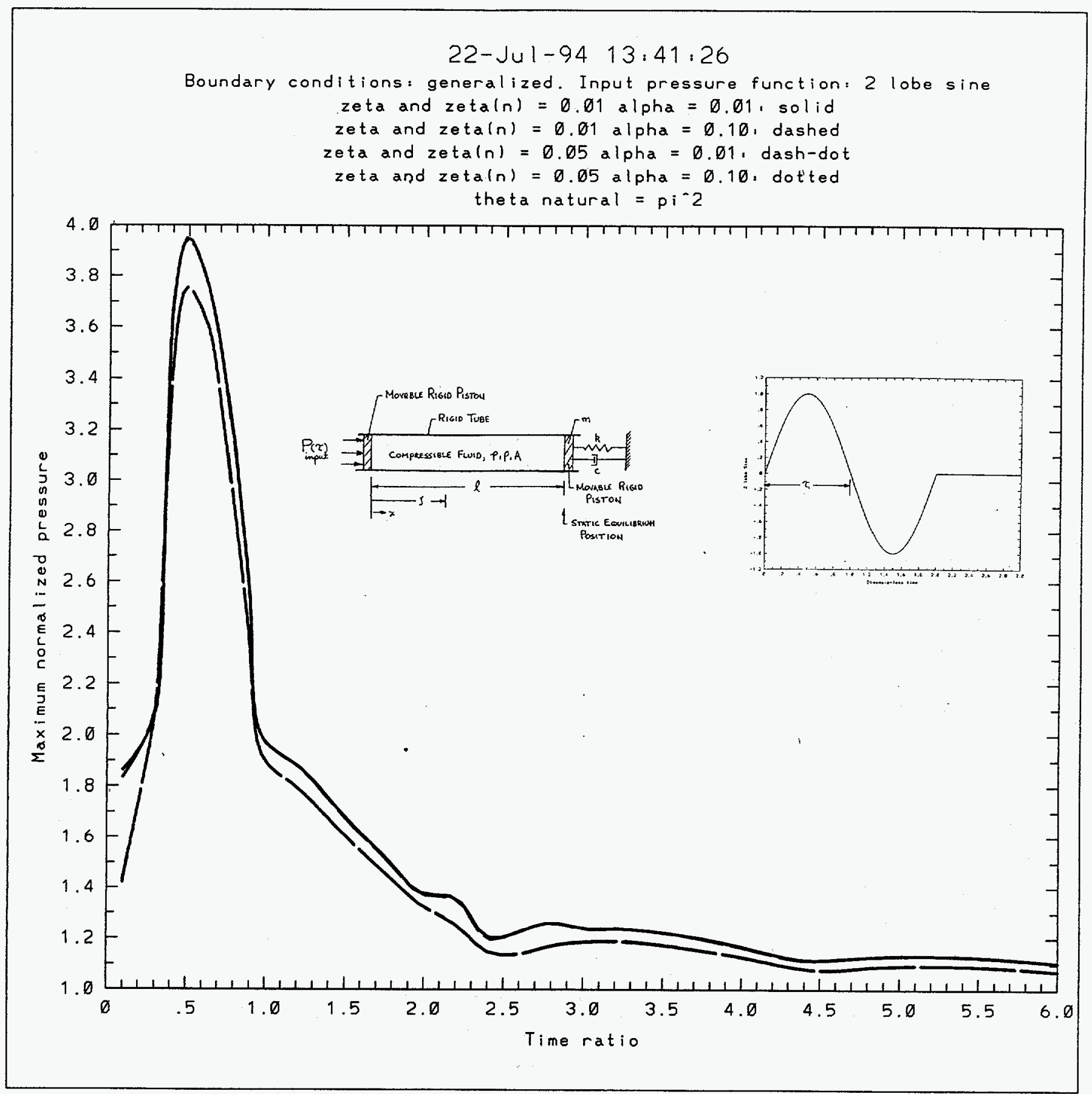

Figure 31 


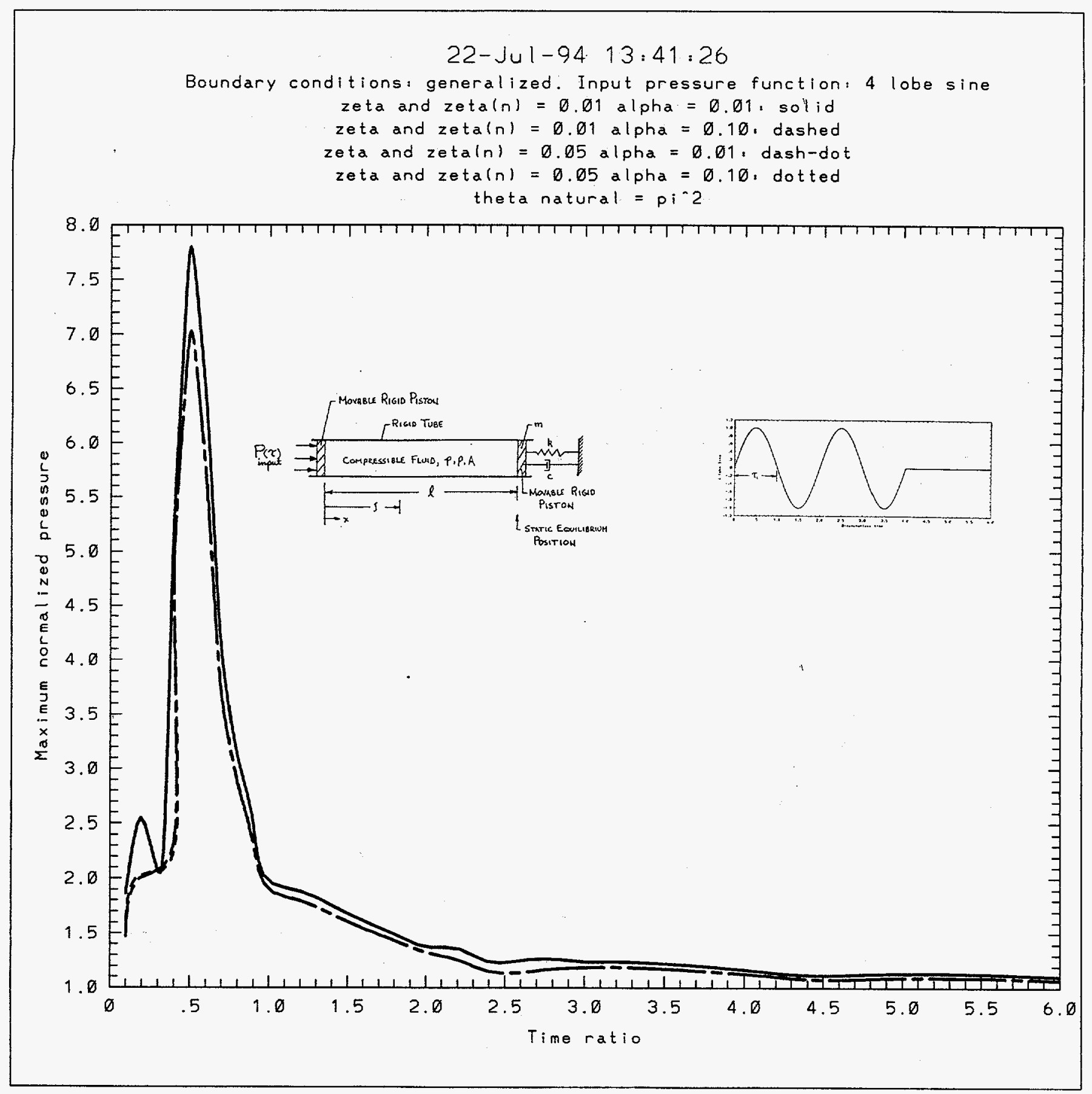

Figure 32 


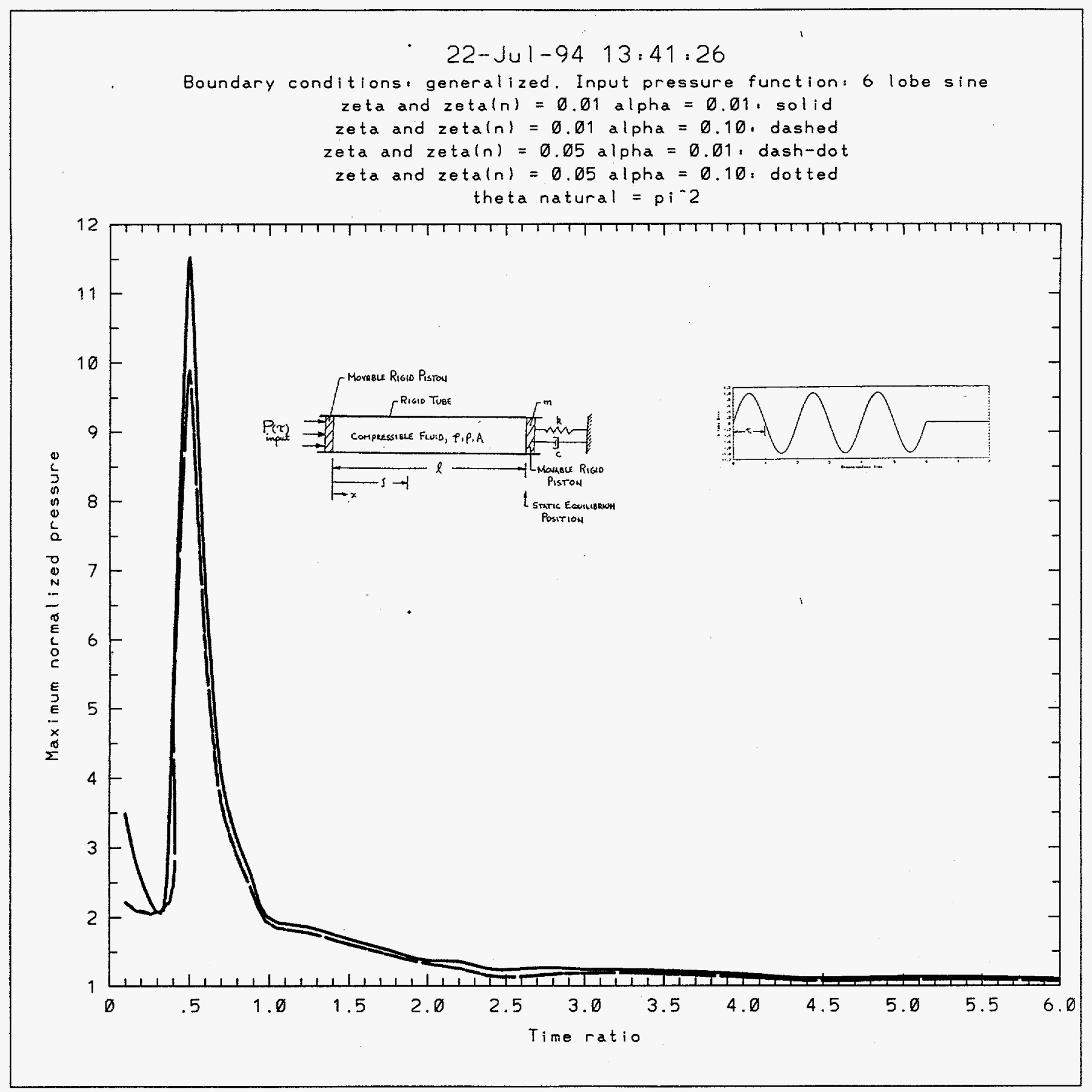

Figure 33 


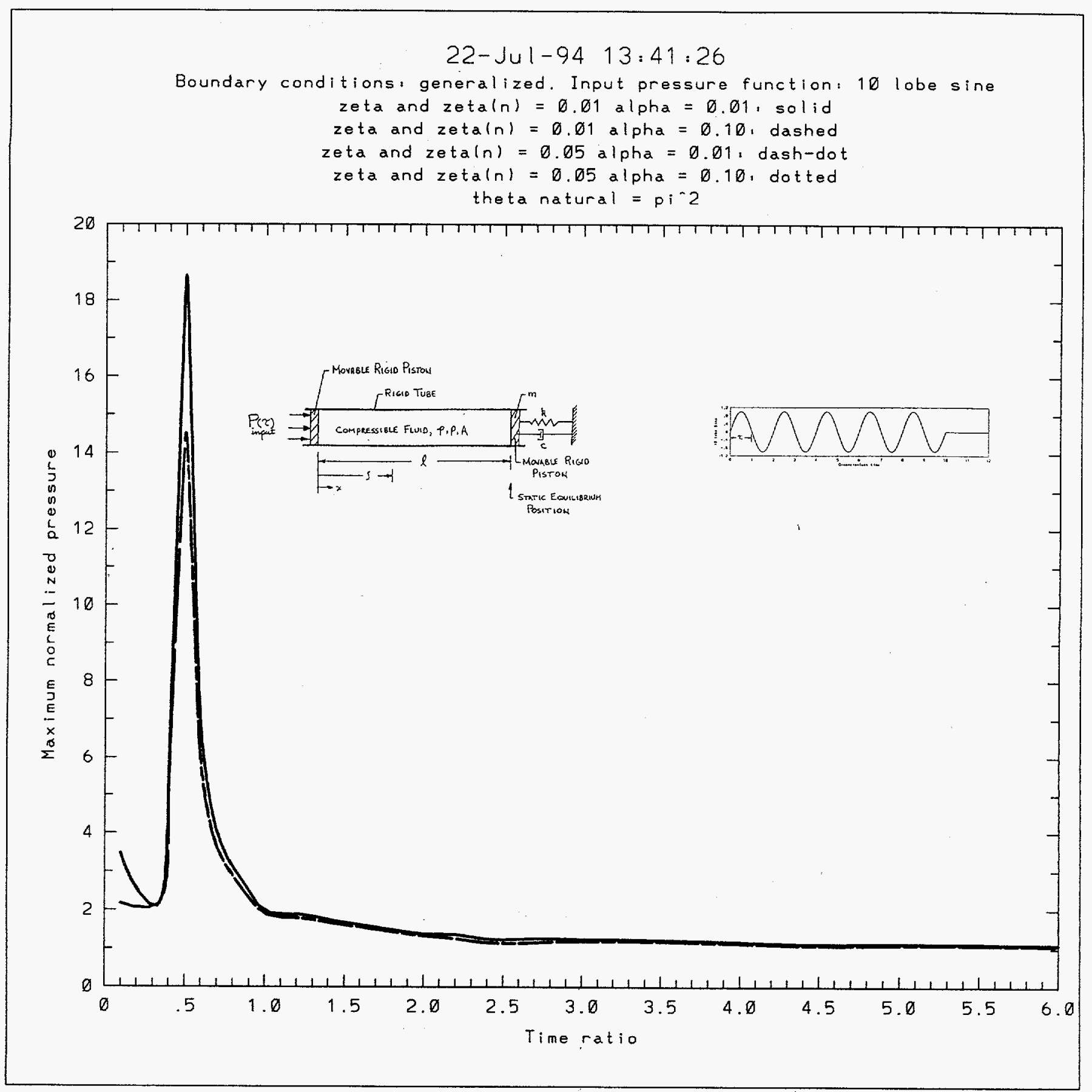

Figure 34 


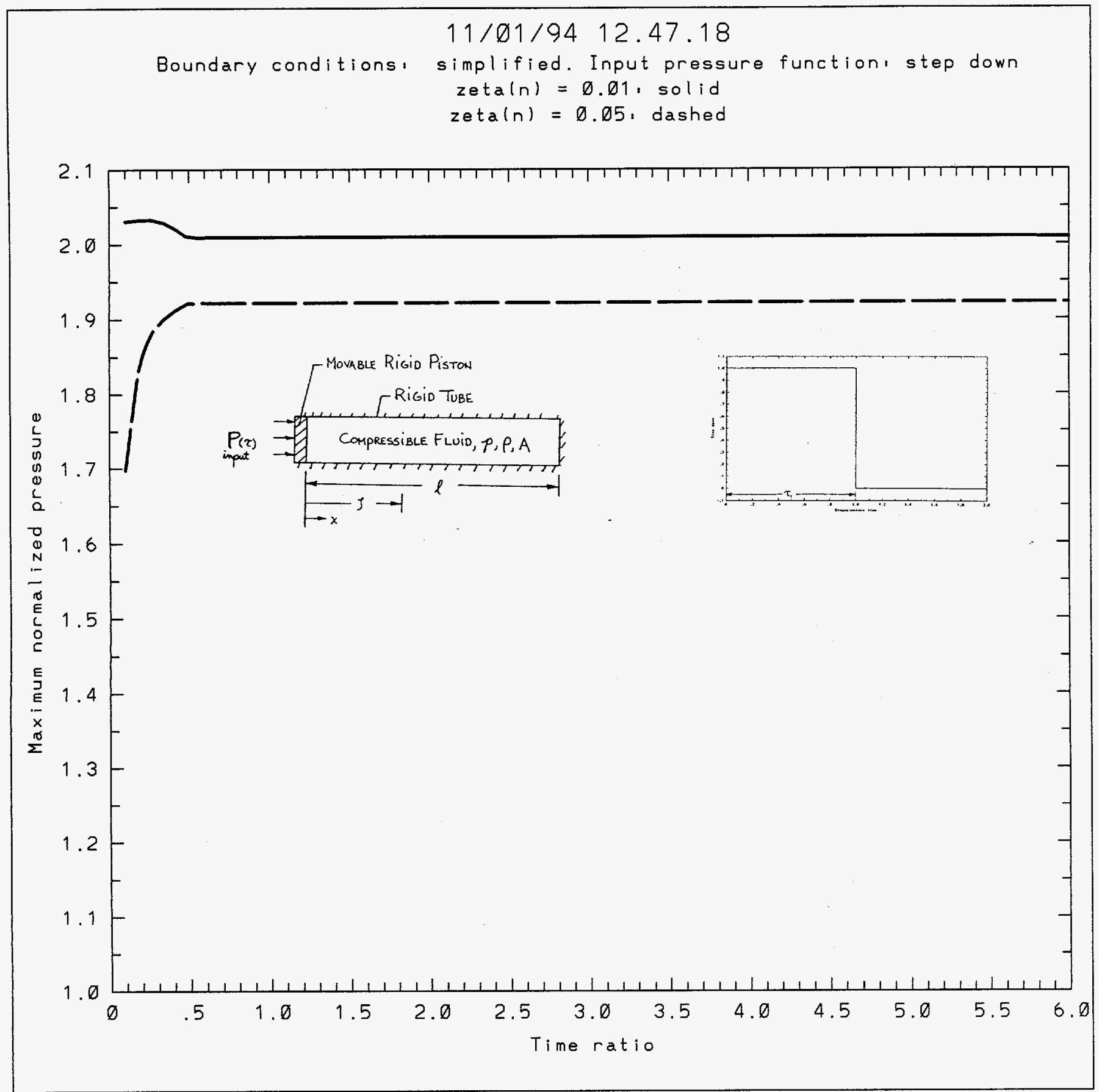

Figure 35 


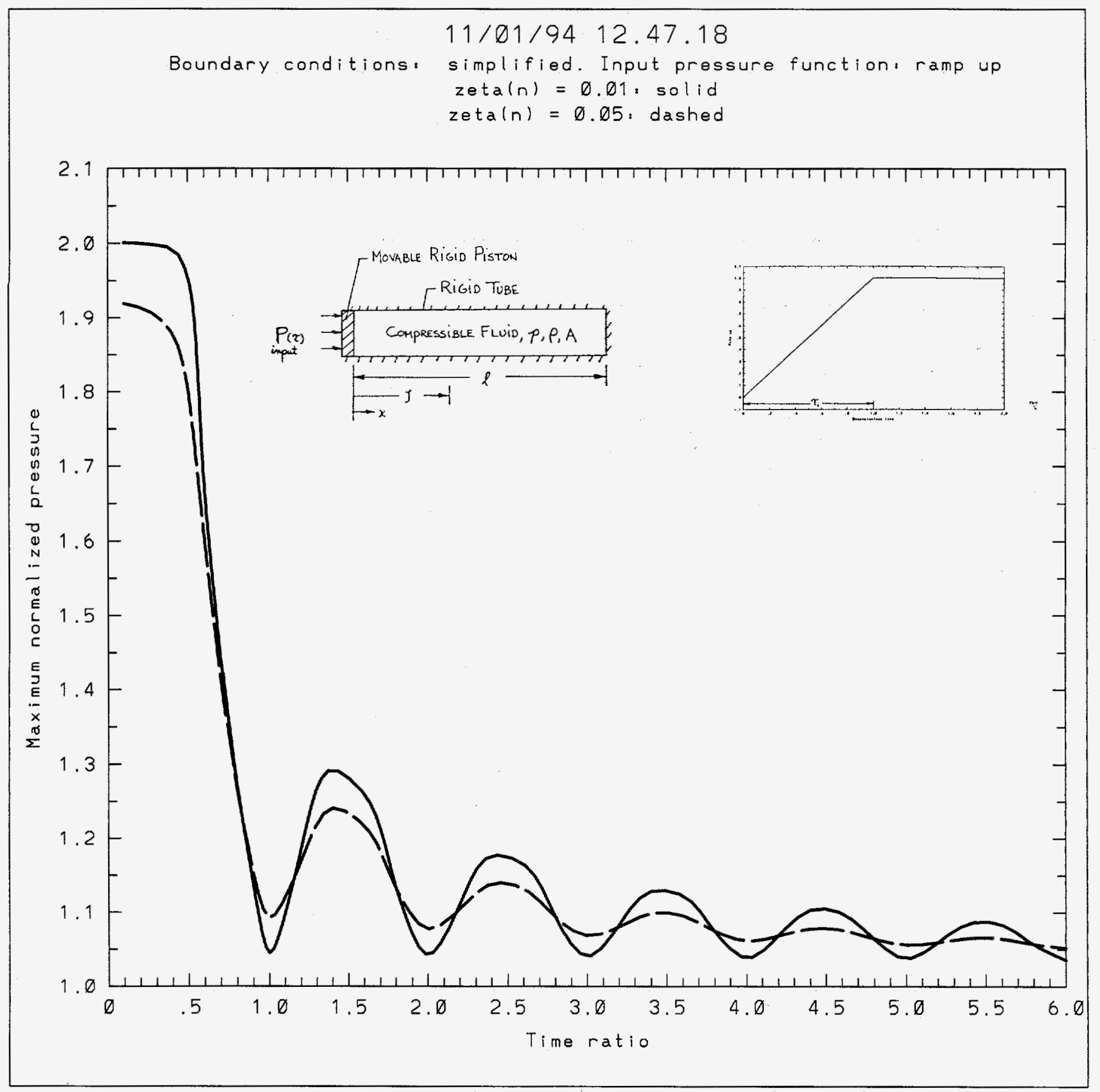

Figure 36 


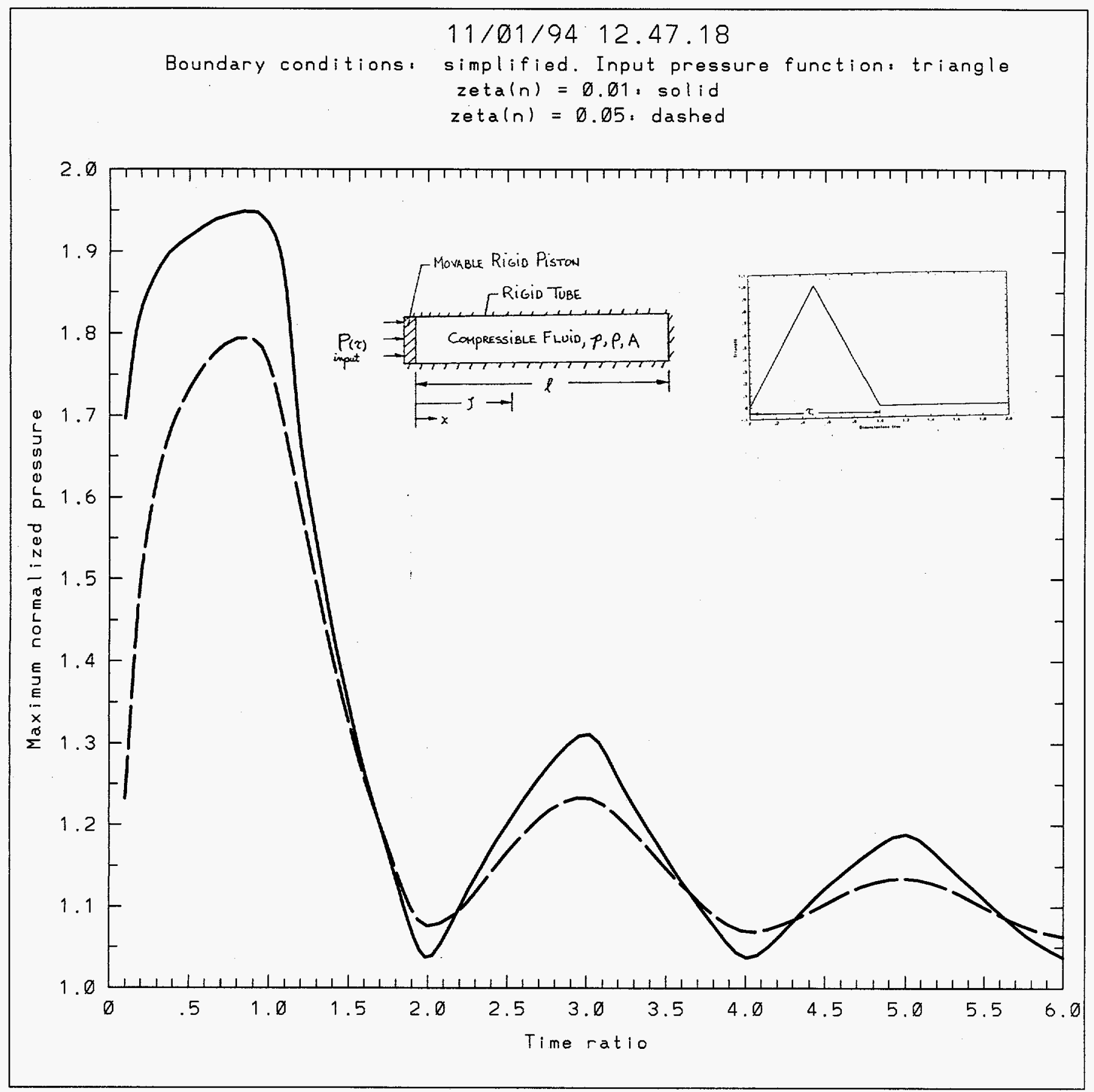

Figure 37 


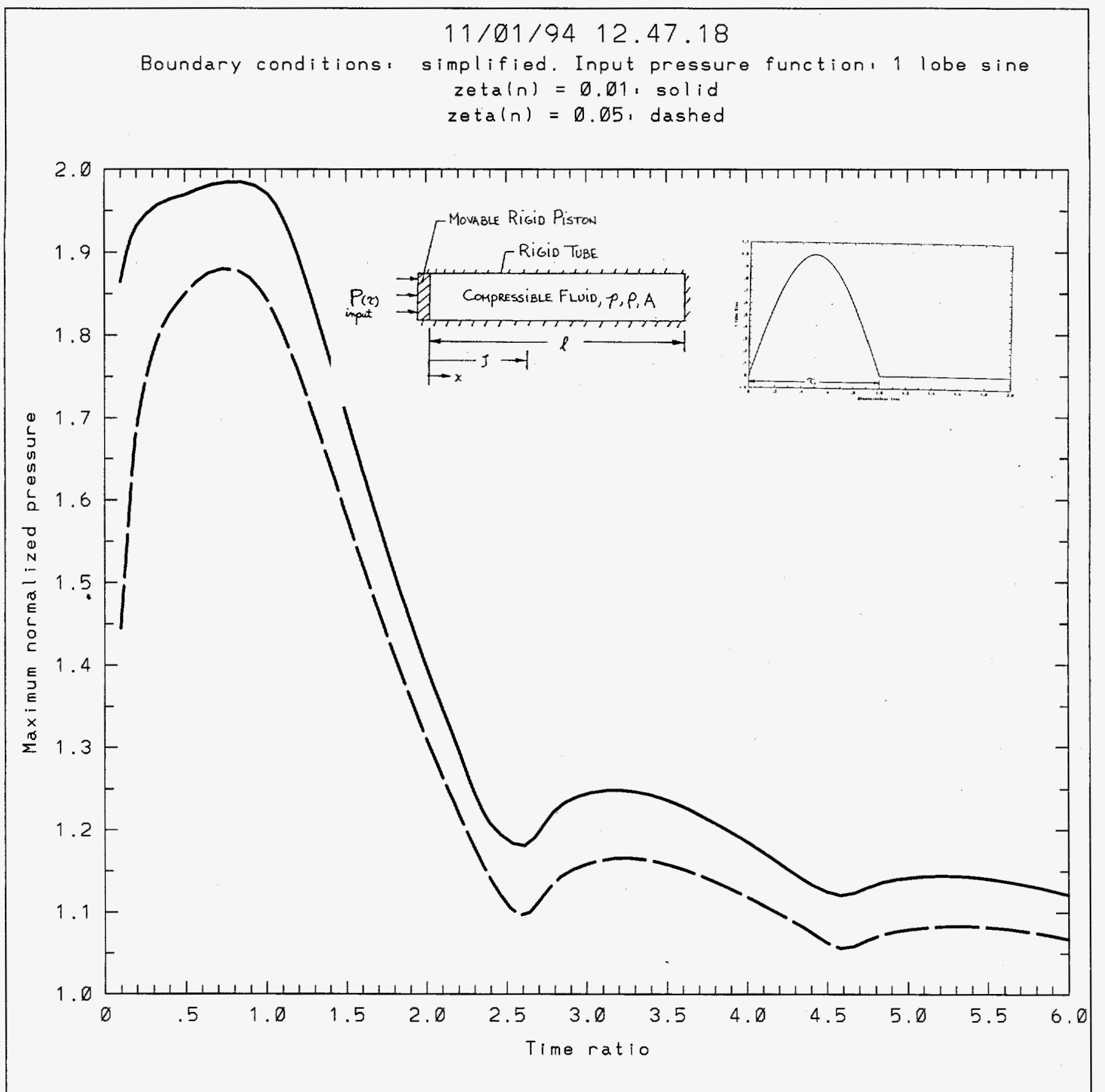

Figure 38 


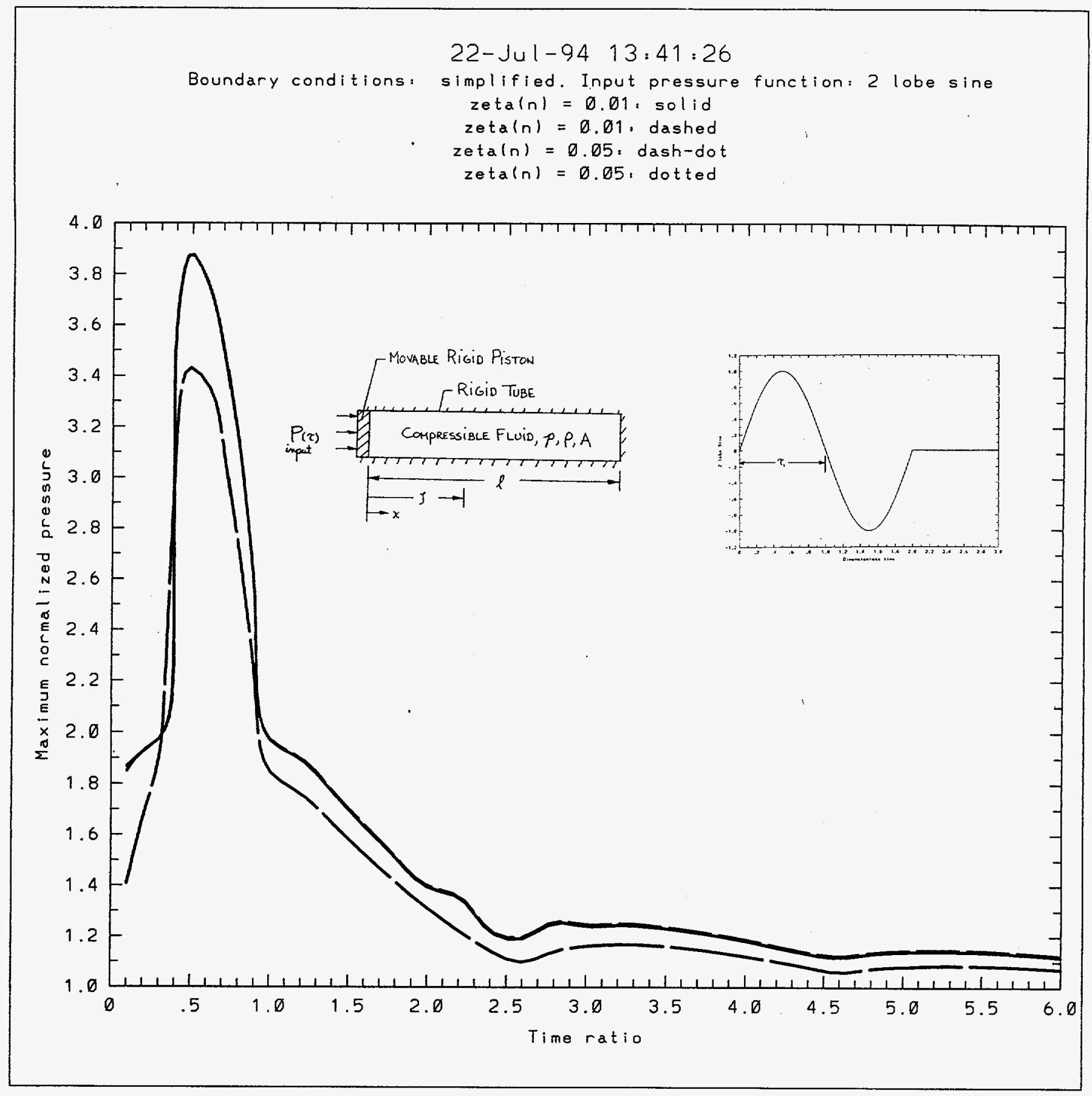

Figure 39 


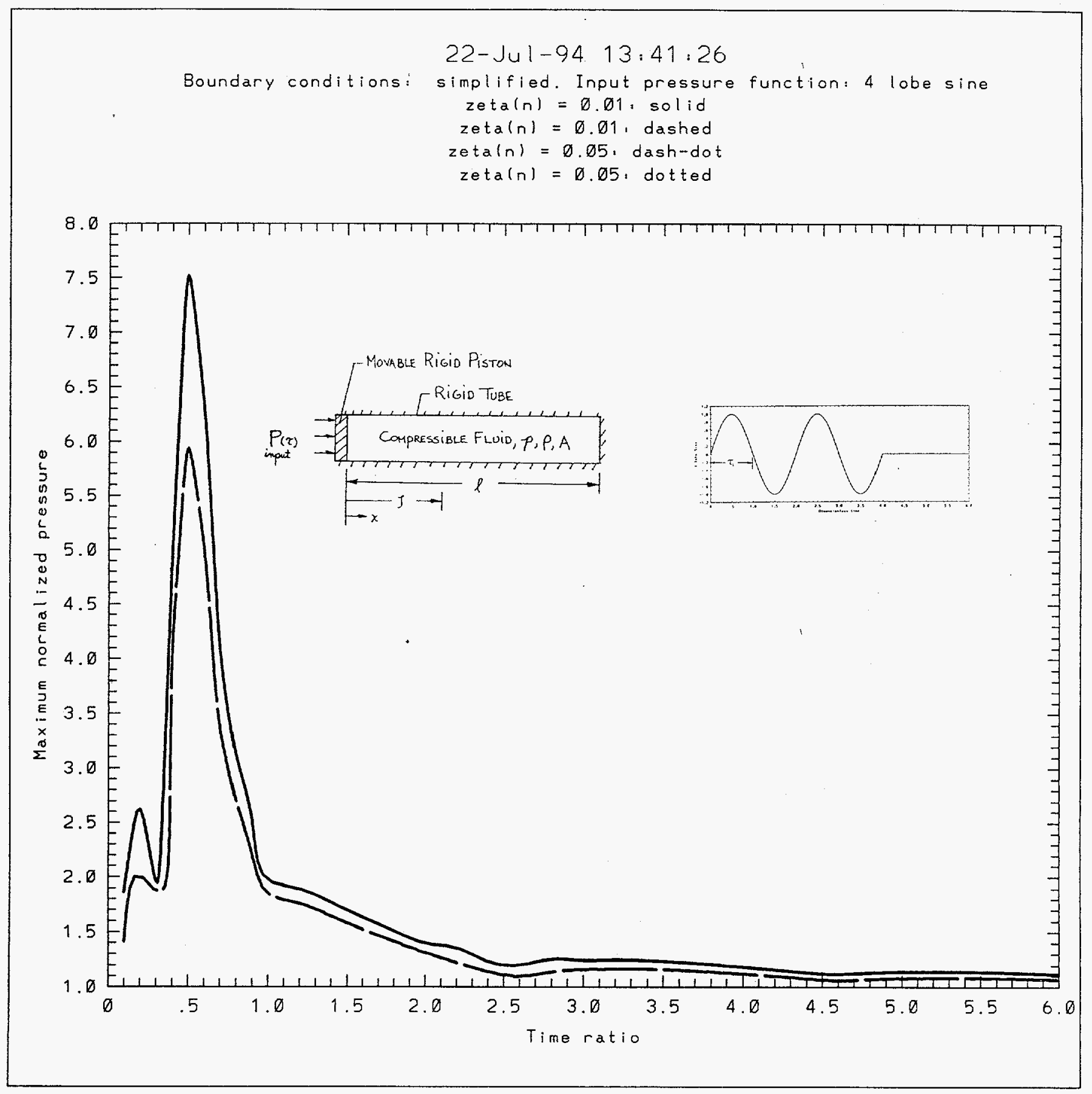

Figure 40 


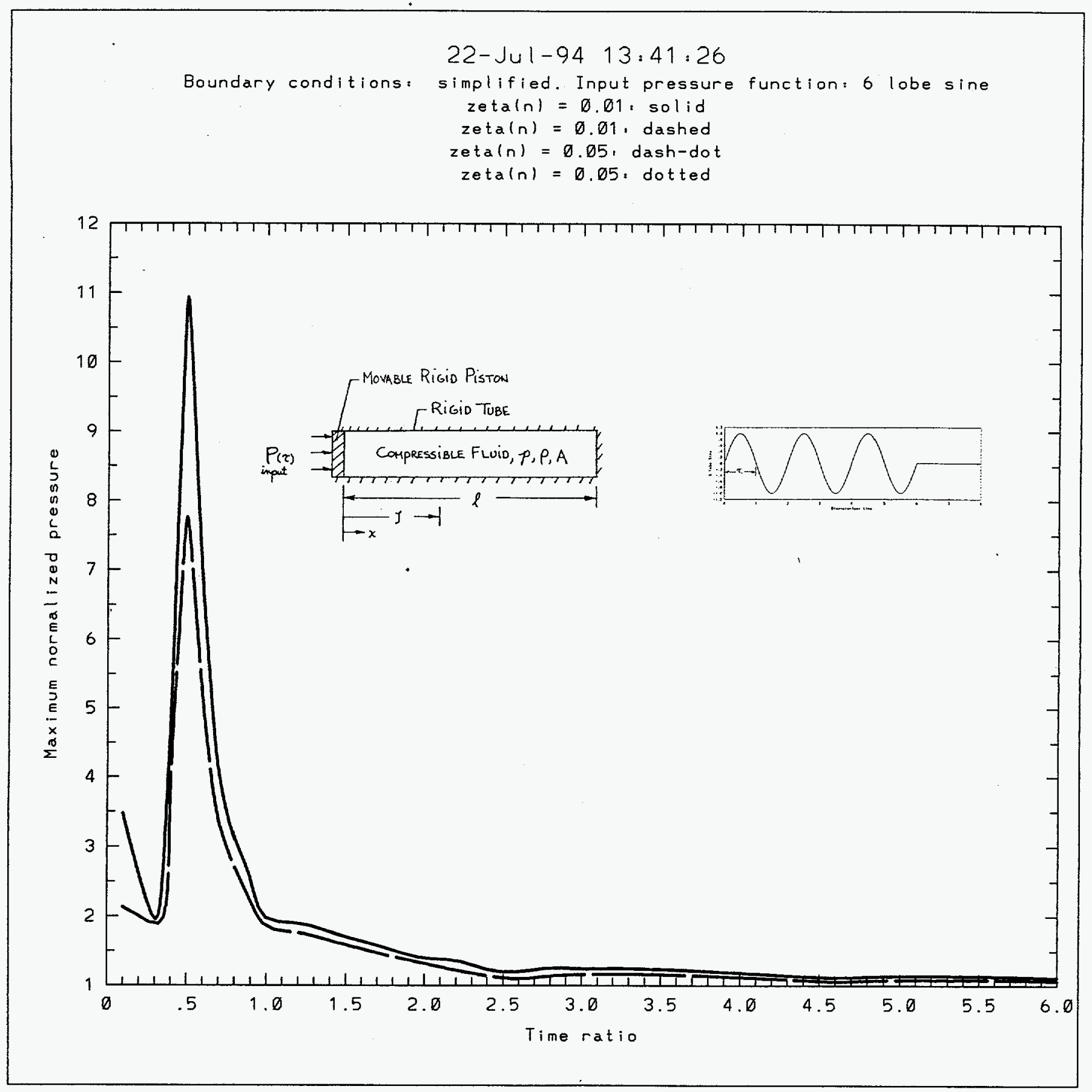

Figure 41 


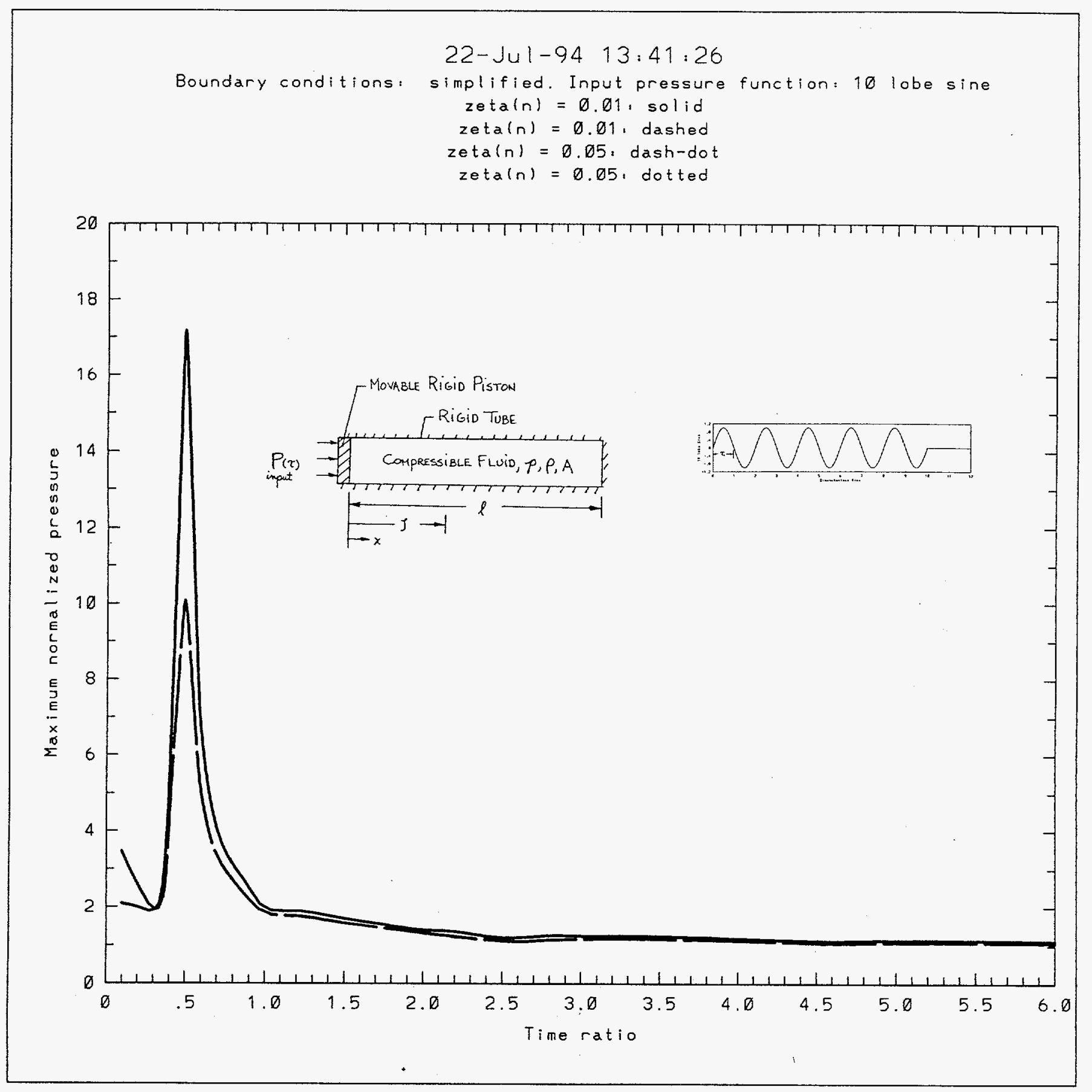

Figure 42 


\section{Numerical Examples}

1. Given a specified input pressure $p(0, t)$ applied to the base of a $155 \mathrm{~mm}$ diameter projectile and expressed by Eq. (62), determine the following when the reference cavity pressure $p_{o}$ is zero.

a. Specified normalized input pressure, $\underset{\text { nor }}{p}(0, \tau)$.

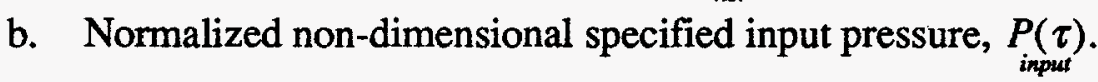

c. Plot $\underset{\text { input }}{P(\tau)}$ versus the non-dimensional time, $\tau$.

d. For $\zeta=0.03, \zeta_{n}=0.03, \alpha=0.01, \theta_{\text {nat }}=\pi$, use Eqs. (41), (40), and (44) to calculate

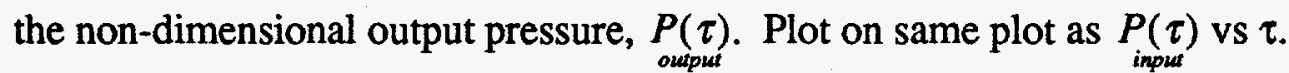

$$
p(0, t)=\alpha t^{4} e^{-\beta t} U(t)
$$

where

$$
\begin{aligned}
& \alpha=p_{\max } / t_{p}^{4} e^{-4} \\
& \beta=4 / t_{p} \\
& U(t)=u(t)-u\left(t-t_{\text {exit }}\right)
\end{aligned}
$$

The expressions for $\alpha$ and $\beta$ result from specifying the maximum pressure when $t=t_{p}$. $\mathrm{U}(\mathrm{t})$ is composed of two unit step functions.

$$
\begin{aligned}
& p_{\max }=50,000 \mathrm{psi} \\
& t_{p}=6.5 \times 10^{-3} \mathrm{secs} \\
& t_{\text {exit }}=14 \times 10^{-3} \mathrm{secs} \\
& c_{o}=38,400 \text { inches } / \mathrm{sec} \\
& \ell=0.060 \text { inches }
\end{aligned}
$$

\section{Part a(1):}

Since $t=\frac{\ell}{c_{0}} \tau$, substitute this expression into Eq. (62). The result is

$$
p(0, \tau)=\frac{p_{\max }}{\tau_{p}^{4} e^{-4}} \tau^{4} e^{-\frac{4}{\tau_{p}} \tau} U(\tau)
$$


where $U(\tau)=u(\tau)-u\left(\tau-\tau_{\text {exit }}\right), \tau_{p}=4,160.0$, and $\tau_{\text {exit }}=8,960.0$.

The specified normalized input pressure is obtained by using Eq. (58) and is expressed by Eq. (64) where $\underset{n o r}{p}(0, \tau)$ has units of pressure. Recall $p_{o}=0$.

$$
\underset{n o r}{p}(0, \tau)=\rho_{o} c_{o}^{2}\left[\frac{\tau^{4}}{\tau_{p}^{4} e^{-4}} e^{-\frac{4}{\tau_{p}} \tau} U(\tau)\right]
$$

\section{$\underline{\text { Part b(1): }}$}

The normalized non-dimensional specified input pressure $\underset{\text { input }}{P(\tau)}$ is obtained by substituting Eq. (64) into Eq. (65).

$$
P_{\text {input }}(\tau)=\frac{1}{\rho_{o} c_{o}^{2}}\left[\underset{\text { nor }}{p}(0, \tau)-p_{o}\right]
$$

Since $p_{o}=0$, this results in Eq. (66).

$$
P_{\text {input }}(\tau)=\frac{\tau^{4}}{\tau_{p}^{4} e^{-4}} e^{-\frac{4}{\tau_{p}} \tau} U(\tau)
$$

Note that when $\tau=\tau_{p}, P(\tau)=1.0$ and that $\underset{\text { input }}{P(\tau)}$ and $\tau$ are dimensionless.

\section{$\underline{\text { Part } c(1)}$ :}

Eq. (66) is plotted in Figure 43. Note that in this particular case there is no dynamic response. However, if the integration time $\tau$ extended beyond $\tau_{\text {exit }}$, a transient dynamic response would occur due to the sudden decrease in pressure at $\tau_{\text {exit- }}$ 


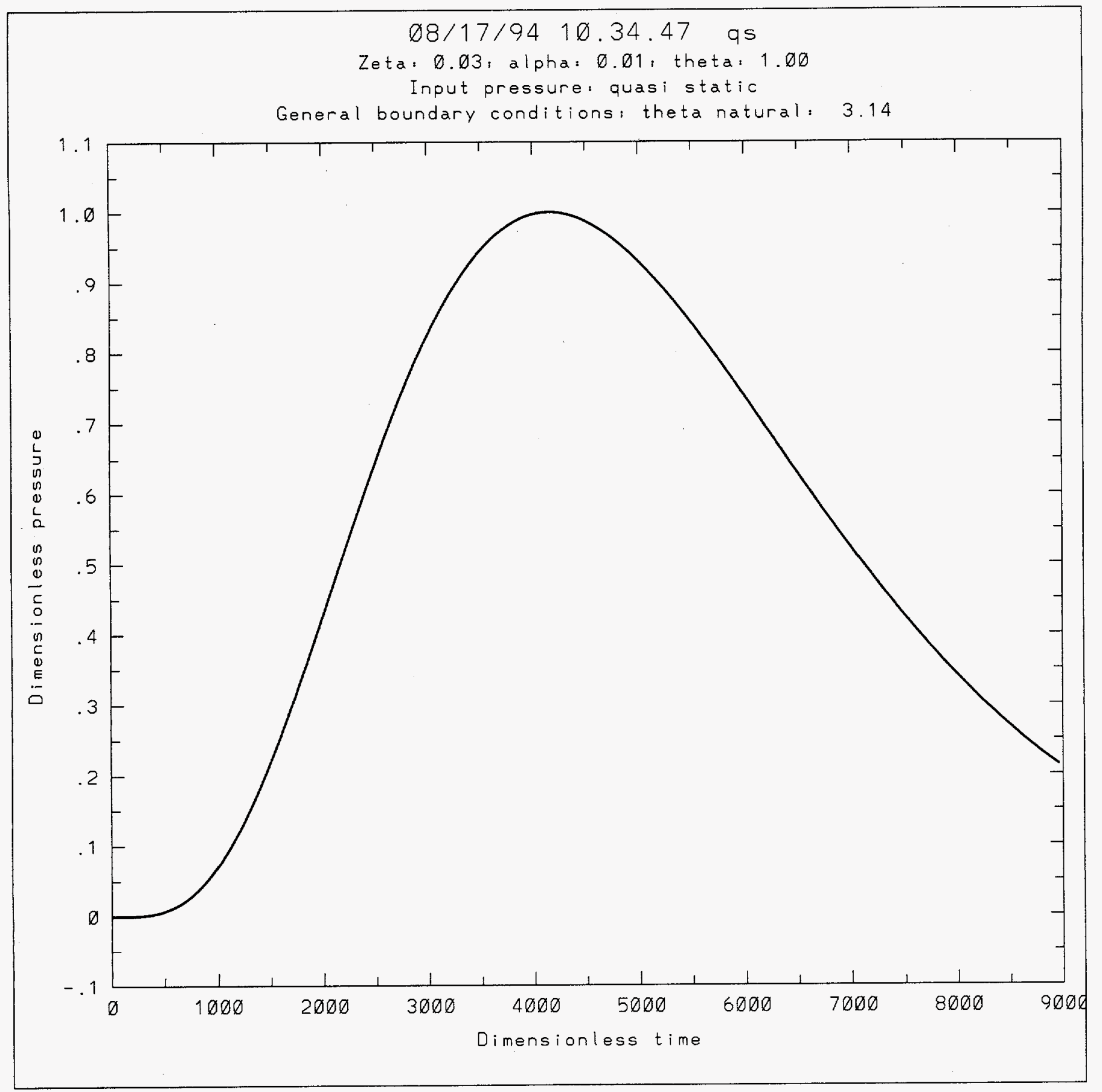

Figure 43 


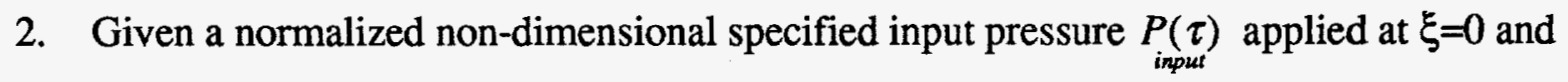
expressed by Eq. (67), determine the non-dimensional output pressure $\underset{\text { ouput }}{P(\tau)}$ at $\xi=1$ (i.e., on the face of the transducer piston) versus the non-dimensional time $\tau$ for the system shown in Figure 1. Note the number of cycles required for steady state vibration.

$$
\underset{\text { input }}{P(\tau)}=\sin \theta \tau
$$

where $\theta=1.0$. The non-dimensional physical system parameters are:

$$
\begin{aligned}
& \alpha=0.01 \\
& \zeta=0.03 \\
& \zeta_{n}=0.03, n=0,1,2,3, \ldots N \\
& \theta_{\text {nat }}=\pi
\end{aligned}
$$

\section{Solution:}

Utilizing Eqs. (41), (40), and (44) results in Figure 44 for $\underset{\text { ouput }}{P(\tau)}$ vs $\tau$. The steady state pressure agrees with the "exact" solution in reference 1 . Repeat the calculation for $\theta=1.50$. The results are shown in Figure 45. 


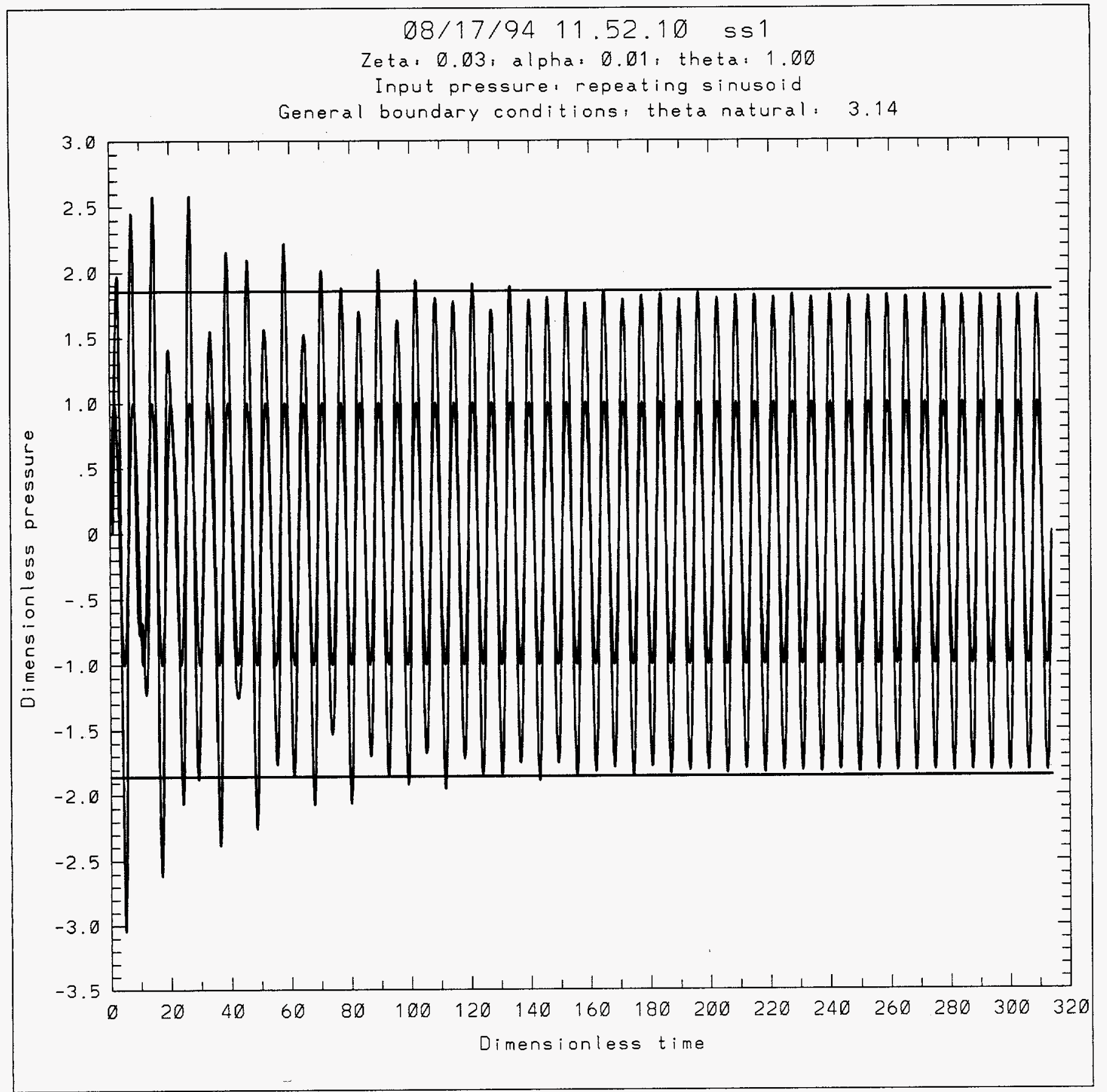

Figure 44 


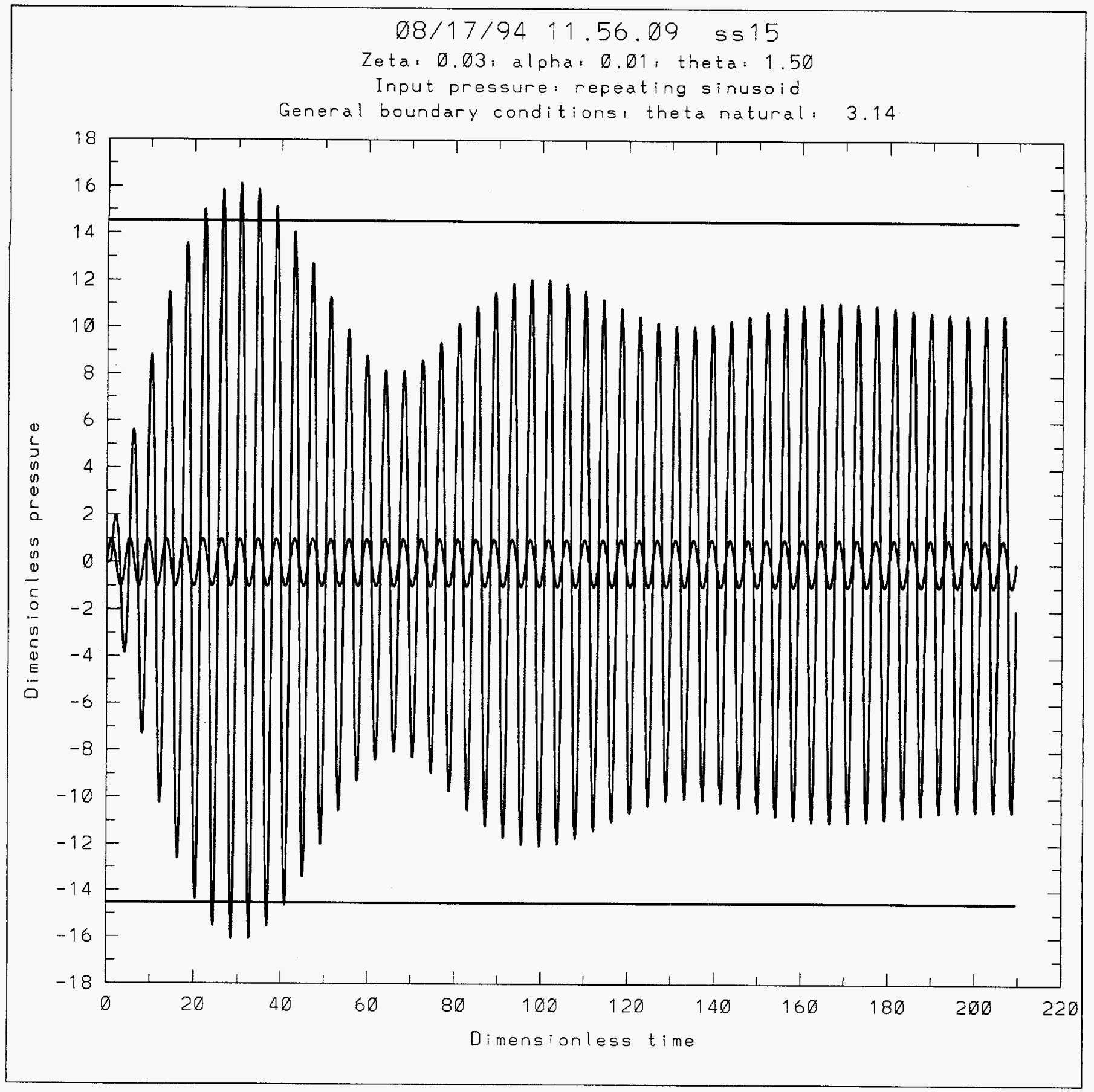

Figure 45 
3. Given a normalized non-dimensional specified input pressure $P(\tau)$ applied at $\xi=0$ and expressed by Eq. (68), determine the non-dimensional output pressure $P(\tau)$ at $\xi=1$ (i.e., on the face of the transducer piston) versus the non-dimensional time $\tau$ for the system shown in Figure 1.

$$
\underset{\text { input }}{P(\tau)}=\sin \theta \tau
$$

where $\theta=\pi / \tau_{1}$ and $P(\tau)=0$ for $\tau>\tau_{1}$ and for $\tau<0$; that is, a half sine pulse. The nondimensional physical system parameters are:

$$
\begin{aligned}
& \alpha=0.10 \\
& \zeta=0.05 \\
& \zeta_{n}=0.05, n=0,1,2,3, \ldots N \\
& \theta_{\text {nat }}=\pi^{2} \\
& \tau_{1}=4.0
\end{aligned}
$$

Solution:

Utilizing Eqs. (41), (40), and (44) results in Figure 46 for $\underset{\text { ouput }}{P(\tau)}$ vs $\tau$. 


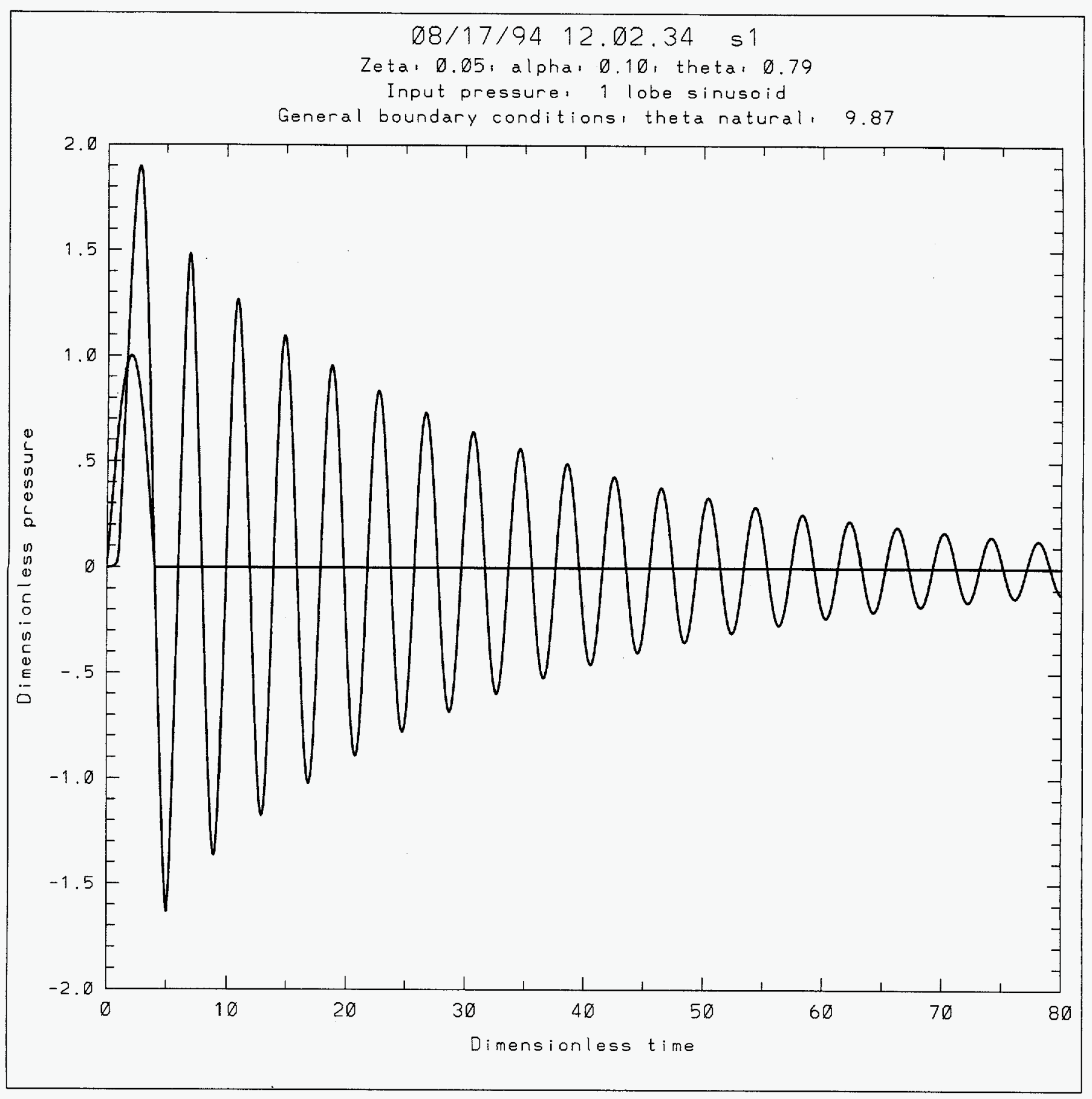

Figure 46 
4. The face of the movable massless piston shown in Figure 1 is subjected to a specified input pressure consisting of a half-sine pressure pulse expressed by Eq. (69).

$$
p(0, t)=p_{\max } \sin \omega t
$$

where

$$
\begin{aligned}
& p_{\max }=3,000 p s i \text { and } p(0, t)=0 \text { for } t>t_{1} \text { and for } t<0 \\
& \omega=\pi / t_{1} \\
& t_{1}=10 \times 10^{-6} \mathrm{secs}
\end{aligned}
$$

The local sound speed $c_{o}=3,200 \mathrm{ft} / \mathrm{sec}$; the one-dimensional cavity length $\ell=0.0244$ inches; the transducer's first natural frequency $\mathrm{f}_{\text {nat }}=250 \mathrm{kHz}$. Also, $\alpha=10 \%, \zeta=5 \%, \zeta_{n}=5 \%$ for $n=0,1,2,3, \ldots N$ and $p_{o}=0$.

\section{Determine:}

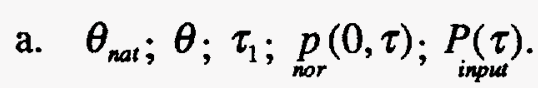

b. Find the maximum normalized pressure (refer to Eq. (55)) and the maximum pressure acting on the face of the transducer located at $x=\ell$.

\section{Solution:}

Part a (4):

$$
\begin{aligned}
& \theta_{\text {nat }}=\omega_{\text {nat }} \frac{\ell}{c_{o}}=2 \pi f_{\text {nat }} \frac{\ell}{c_{o}} \\
& \theta_{\text {nat }} \cong 1.0 \\
& \theta=\omega \frac{\ell}{c_{o}}=\frac{\pi}{t_{1}} \frac{\ell}{c_{o}} \\
& \theta \cong 0.2
\end{aligned}
$$

Note that $f=\frac{\theta}{2 \pi} \frac{c_{o}}{\ell}=50 \mathrm{kHz}$ and that the wave length $\lambda=\frac{c_{o}}{f}=0.768$ inches. 
$\tau_{1}=\frac{c_{o}}{\ell} t_{1}=15.74$

Since $p_{o}=0$ and $\left|p\left(0, \frac{c_{o}}{\ell} t\right)\right|_{\max }=3,000$ psi, $\underset{\text { nor }}{p}(0, \tau)$ is: (refer to Eq. (58))

$\underset{\text { nor }}{p}(0, \tau)=\rho_{o} c_{o}^{2}[\sin \theta \tau]$ and $\underset{\text { nor }}{p}(0, \tau)=0$ for $\tau>\tau_{1}$ and for $\tau<0$

$\underset{\text { input }}{P(\tau)}$ is: (refer to Eq. (41a))

$\underset{\text { input }}{P(\tau)}=\sin \theta \tau$ and $\underset{\text { input }}{P(\tau)}=0$ for $\tau>\tau_{1}$ and for $\tau<0$

\section{$\underline{\text { Part b(4): }}$}

Recall that from Eq. (61), $T_{1}=4$.

$$
\frac{\tau_{1}}{T_{1}}=3.94
$$

From Figure 14:

$$
\frac{\left|\begin{array}{l}
P(\tau) \\
\text { oupput }
\end{array}\right|_{\max }}{\left.|P(\tau)|_{\text {input }}\right|_{\max }} \cong 1.12 \text { (i.e., a } 12 \% \text { error) }
$$

The maximum pressure acting on the face of the linear transducer is:

$$
|p(\ell, t)|_{\max }=\left.\frac{\left|\begin{array}{l}
P(\tau) \\
\text { oupui }
\end{array}\right|}{\left|\begin{array}{l}
\max \\
\text { input }
\end{array}\right|}\right|_{\max } p_{\max }=(1.12)(3,000 .)=3,360 . \mathrm{psi}
$$

Recall that $p(0, t)$ is normalized by dividing by $p_{\max }$ to obtain $\underset{n o r}{p}(0, \tau)$. Due to the dynamic response of the system, the transducer "measured" a maximum output pressure $12 \%$ higher than the maximum input pressure. 


\section{Conclusions And Discussion}

A mathematical model for a pressure transducer mounted in a fluid filled cavity (a system) is developed and the pressure "measurement" error of the cavity and transducer is determined by computing the dynamic response (output pressure) of the system to a specified pressure time history (input pressure). The "measurement" error is determined by comparing the calculated output pressure to the specified input pressure. Consequently, the non-dimensional equations and associated computer code, which describe the dynamic behavior of the pressure measurement system, can be used to determine pressure "measurement" errors for measurement systems whose physical system parameters and input pressures are different from those considered in this report.

The results obtained in this report are useful in determining the dynamic response (transient and steady state) of a pressure measurement system such as illustrated in Figure 1. Pressure shock response spectra are determined for a variety of input pressure time functions. These spectra are useful in determining if the magnitude of certain input pressure time histories are significantly altered (i.e., amplified or attenuated, which is a measurement error) by the pressure transducer cavity and/or the transducer. Also examined is the important and practical case where the natural frequency of the transducer is much higher than the first natural frequency of the cavity, and the first natural frequency of the cavity is much higher than the highest "measured" input frequency, i.e., $\theta_{\text {nat }} \gg \theta_{1}$ and $\theta_{1} \gg \theta_{\max }$.

The section on Results provides extensive pressure response spectra plots over a reasonably large physical system parameter space for a moderate number of specified input pressure time histories. Since solutions to the non-dimensional equations of motion are used to generate the non-dimensional response spectra, the response spectra plots apply to any onedimensional acoustic cavity with the same non-dimensional physical system parameters. Numerical examples are also provided to illustrate the use of the pressure response spectra plots.

A major conclusion: for the non-dimensional physical system parameters shown on the response spectra plots and for input pressure time histories which are ramped, triangular, or sinusoidal where the time duration of the input $\tau_{D}$ is an integer multiple of the half period $\tau_{1}$ of the sinusoid (i.e., $\tau_{D}=n \tau_{1}$, where $n=1,2,3, \ldots . N$ ), the maximum measurement error between the maximum output and maximum input pressures (i.e., maximum normalized pressure) is less than or equal to about 20 to $25 \%$ when the time ratio is equal to or greater than 2.5 . That is, when 


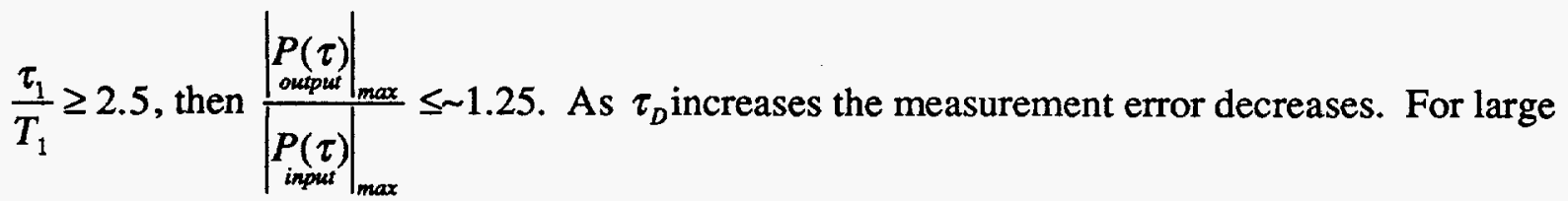
values of $\frac{\tau_{1}}{T_{1}}$ and $\tau_{D}$, the measurement error approaches zero. When the time ratio $\frac{\tau_{1}}{T_{1}}$ is much less than 2.5, very large pressure measurement errors occur. This results because the highest "measured" input frequency associated with the oscillatory input pressure is close to or greater than the first natural frequency of the transducer cavity.

If a comparison of the output pressure to the input pressure with respect to time is of interest, and this is often the case, it is necessary to numerically integrate Eqs. (41) for the appropriate initial conditions [e.g., refer to Eqs. (42)] and determine the output pressure from Eqs. (40) and (44).

Under certain conditions, the time dependent boundary condition associated with the transducer can be replaced with reasonable accuracy by the boundary condition associated with a rigid wall. For this case the equations of motion uncouple and an analytical solution is obtained in the form of an integral which can be evaluated by numerical quadrature.

Appendix A contains examples of the early and long time dynamic response of the nondimensional output pressure at $\xi=1[$ i.e., $\underset{\text { output }}{P(\tau)}$ vs $\tau]$ for several normalized non-dimensional specified input pressures applied at $\xi=0[$ i.e., $\underset{\text { input }}{P(\tau)}$ vs $\tau]$. The early time response plots, Figures A-1 through A-9, result from numerically integrating Eqs. (41) for the initial conditions given by Eqs. (42). $P(\tau)$ istput is then determined from Eqs. (40) and (44). It is worthwhile to examine the time response curves in Appendix A since a single point on the response spectrum plot is determined from a single point (the maximum response) on the time response curve and the time response curves look completely different than the pressure response spectra plots. Recall that each point on a pressure response spectrum plot requires the generation of a pressuretime response curve. Consequently, the generation of a pressure response spectrum curve or plot represents a significant amount of calculation or computer time.

The amplitude modulated transient response shown in Figure A-10 results because Eqs. (41) are highly coupled (i.e., $\alpha=0.01$ and $\theta_{\text {nat }}=\pi$ ) and many of the lower modal frequencies 
differ only slightly from each other. Consequently, during the slightly damped transient vibration, energy is transferred between the lower modes and this is observed in Figure A-10 as an amplitude modulated transient vibration. Since the transient dynamic response in Figure A-10, which consists of oscillations at the damped natural frequencies, damps out or disappears as $\tau$ increases indefinitely, the dynamic response of the system becomes a steady state harmonic oscillation of non-dimensional circular frequency $\theta$ and the amplitude of the steady state oscillation depends on the non-dimensional physical system parameters.

References 1 and 2 show that stepped-recessed pressure transducer cavities, which may be grease-filled, are suitable for measuring quasi-static pressure in solid propellant guns and regenerative liquid propellant guns, provided the frequencies contained in the quasi-static pressure pulse are low relative to the fundamental frequency for the empty stepped cavity.

Accurately measured oscillatory pressure-time histories are required to determine the dynamic structural response for projectiles (as well as for guns) and to assess their structural integrity with respect to oscillatory pressure environments in regenerative liquid propellant guns, RLPGs. Unfortunately, grease-filled two diameter cavities or stepped-recessed ports, which have a low fundamental frequency relative to most of the measured frequencies contained in the oscillatory pressures, have been used to measure oscillatory pressures in regenerative liquid propellant guns for many years, and this has resulted in an inaccurate oscillatory pressure data base over a large region of the frequency range of interest. Analysis and laboratory experiments both confirm this. Further, the issue of using grease-filled stepped-recessed transducer ports versus through-hole transducer ports to measure oscillatory pressures has been ongoing for about three years and apparently is still not totally resolved. There is ample data which shows that grease-filled stepped-recessed cavities are not suitable for measuring oscillatory pressures over a large region of the frequency range of interest. Therefore, it is strongly recommended that this issue be resolved so that the structural integrity of projectiles (as well as regenerative liquid propellant guns) in the inventory can be accurately assessed when subjected to the oscillatory pressure environment associated with RLPGs [Refs. 1 and 2].

\section{Additional Comments Regarding Measurement Error Associated with the Frequency of a Sinusoidal Input Pressure}

The purpose of this section is to briefly point out that accurate high frequency oscillatory pressure measurements depend not only on the transducer's cavity configuration, frequency response of the measurement system, etc., but can also depend on the diameter of the transducer's sensing element. 
When making oscillatory pressure measurements, the following should be noted. Consider a flush mounted transducer and let the velocity vector of the sinusoidal pressure wave be parallel to the circular sensing face of the pressure transducer. That is, the pressure wave sweeps across the face or diameter of the transducer. If the wave length $\lambda$ of the sinusoidal pressure wave is $n d$, where $n$ is an integer number and $d$ is the diameter of the transducer's sensing face, what are the approximate measurement errors for the maximum oscillatory pressure associated with the various values of $n$ and what are the corresponding frequencies?

\section{Derivation and Results}

\section{Consider Figure 47:}

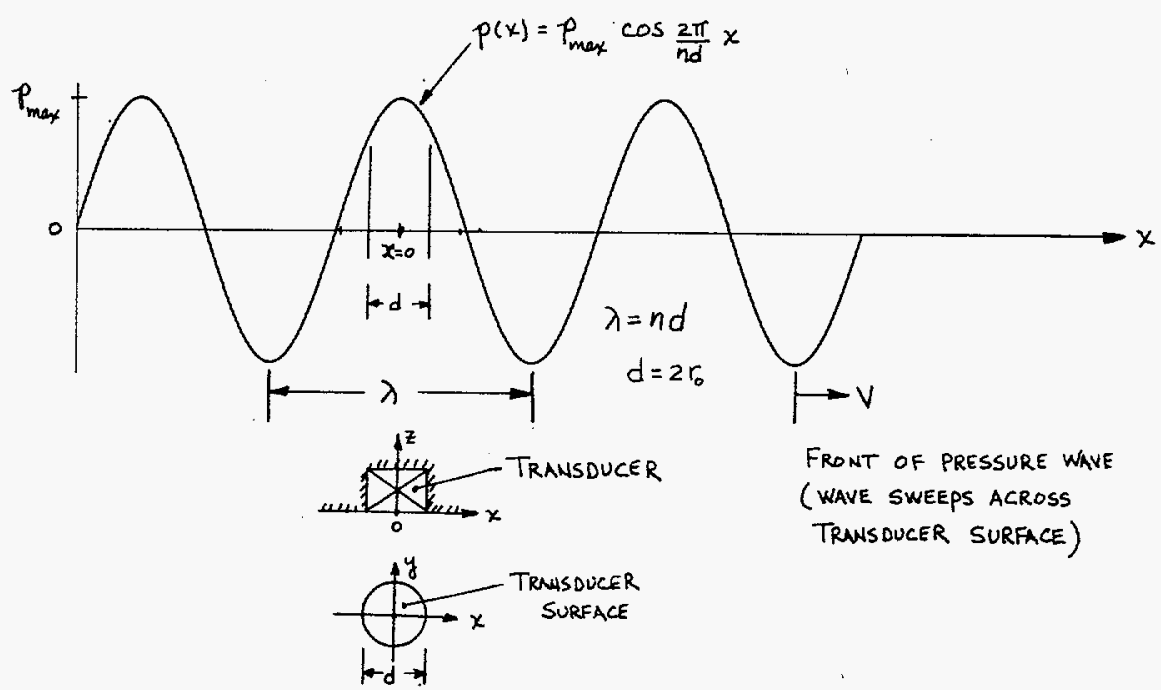

The measurement error due to the frequency of pressure oscillation is obtained by assuming $p=p(y)=$ constant and $p=p(x)$ only. For this case:

$$
\begin{aligned}
& p(t)=p_{\max } \cos \omega t \\
& \omega=2 \pi f \\
& f=c_{o} / \lambda \\
& t=x / c_{o} \\
& \lambda=n d
\end{aligned}
$$

Therefore, 


$$
p(x)=p_{\max } \cos \frac{2 \pi}{n d} x
$$

The force acting on the transducer face is:

$$
F=\int_{A} p(x) d A
$$

or

$$
F=4 p_{\max } r_{o} \int_{x=0}^{x=r_{0}}\left[1-\left(\frac{x}{r_{0}}\right)^{2}\right]^{\frac{1}{2}} \cos \frac{\pi}{n} \frac{x}{r_{0}} d x
$$

Let $x / r_{o}=z$ and $\pi / n=a$, then:

$$
\begin{aligned}
& F=4 p_{\max } r_{0}^{2} \int_{z=0}^{z=1}\left[1-z^{2}\right]^{\frac{1}{2}} \cos a z d z=4 p_{\max } r_{0}^{2} \frac{\pi}{2 a} J_{1}(a) \\
& F=2 p_{\max } r_{0}^{2} n J_{1}(\pi / n)
\end{aligned}
$$

where $J_{1}$ is a Bessel function of the first kind and first order. For the special case where the pressure is uniform (i.e., $\lambda \rightarrow \infty$ and therefore $n \rightarrow \infty$ ), $F_{\max }$ is given by Eq. (71).

$$
F_{\max }=p_{\max } \pi r_{o}^{2}
$$

Dividing Eq. (70) by Eq. (71) results in Eq. (72).

$$
\begin{aligned}
& \frac{F}{F_{\max }}=2 \frac{n}{\pi} J_{1}\left(\frac{\pi}{n}\right) \\
& \% \text { error }=\left[1-\frac{F}{F_{\max }}\right] \times 100
\end{aligned}
$$

or

$$
\% \text { error }=\left[1-2 \frac{n}{\pi} J_{1}\left(\frac{\pi}{n}\right)\right] \times 100
$$

and

$$
f=c_{o} / n d
$$


Table 1a lists the measurement errors associated with various values of $n$. Table $1 \mathrm{~b}$ lists the associated frequencies for various values of $n$ and $c_{o}$ and for $\mathrm{d}=0.25$ inches. Note that the errors for the measured maximum oscillatory pressure are quite large for the higher frequencies (i.e., small values of $n$ ) of pressure oscillation; e.g., $n=2,3$. 


\section{Table 1a}

Measurement Error for Max. Oscillatory Pressure

Due to Frequency of Pressure Oscillation

(Pressure Wave Sweeps Across Transducer Face)

\begin{tabular}{|c|c|c|c|c|c|}
\hline $\mathbf{n}$ & $\mathrm{pi} / \mathrm{n}$ & $\mathrm{J} 1(\mathrm{pi} / \mathrm{n})$ & $F / F \max$ & $1-F / F \max$ & \% Error \\
\hline 2 & 1.5708 & 0.5668 & 0.7217 & 0.2783 & 27.8297 \\
\hline 3 & 1.0472 & 0.4550 & 0.8690 & 0.1310 & 13.0956 \\
\hline 4 & 0.7854 & 0.3632 & 0.9249 & 0.0751 & 7.5150 \\
\hline 5 & 0.6283 & 0.2989 & 0.9515 & 0.0485 & 4.8543 \\
\hline 6 & 0.5236 & 0.2529 & 0.9661 & 0.0339 & 3.3880 \\
\hline 7 & 0.4488 & 0.2188 & 0.9750 & 0.0250 & 2.4967 \\
\hline 8 & 0.3927 & 0.1926 & 0.9808 & 0.0192 & 1.9153 \\
\hline 9 & 0.3491 & 0.1719 & 0.9848 & 0.0152 & 1.5154 \\
\hline 10 & 0.3142 & 0.1551 & 0.9877 & 0.0123 & 1.2286 \\
\hline 11 & 0.2856 & 0.1413 & 0.9898 & 0.0102 & 1.0161 \\
\hline 12 & 0.2618 & 0.1298 & 0.9915 & 0.0085 & 0.8543 \\
\hline
\end{tabular}

where $\frac{F}{F_{\max }}=2 \frac{n}{\pi} J_{1}\left(\frac{\pi}{n}\right)$. 
Table 1b

Frequency of Oscillatory Pressure for Various Values of $n$ and $c_{o}$ $(d=0.25$ inches)

\begin{tabular}{|c|c|c|}
\hline$n$ & $\begin{array}{c}f \\
(\mathrm{kHz}) \\
\text { for } c_{o}=13,333 \text { inches/sec }\end{array}$ & $\begin{array}{c}f \\
(\mathrm{kHz}) \\
\text { for } c_{o}=40,000 \text { inches } / \mathrm{sec}\end{array}$ \\
\hline 2 & 26.6 & 80 \\
\hline 3 & 17.8 & 53.3 \\
\hline 4 & 13.3 & 40 \\
\hline 5 & 10.7 & 32 \\
\hline 6 & 8.9 & 26.7 \\
\hline 7 & 7.6 & 22.8 \\
\hline 8 & 6.7 & 20 \\
\hline 9 & 5.9 & 17.8 \\
\hline 10 & 5.3 & 16 \\
\hline 11 & 4.8 & 14.5 \\
\hline 12 & 4.4 & 13.3 \\
\hline
\end{tabular}

Correspondingly, if the frequency of the oscillatory pressure is $50 \mathrm{kHz}$ and the error in the measured oscillatory pressure is not to exceed $4.85 \%$ (i.e., $\lambda=n d$ where $n=5$ ), what is the diameter $d$ of the transducer's sensing face when $c_{o}=40,000$ inches $/ \mathrm{sec} . ?$

$$
\begin{aligned}
& d=\frac{c_{o}}{n f} \\
& d=\frac{40 \times 10^{3}}{5 \times 50 \times 10^{3}} \cong 0.160 \text { inches } \\
& d \cong 0.160 \text { inches }
\end{aligned}
$$


From examining Eqs. (73a) and (73b) and Table 1, it is apparent that accurate high frequency oscillatory pressure measurements depend on the diameter of the transducer's sensing element provided the pressure wave sweeps across the face of the transducer. This may affect the selection of the pressure transducers mounted, for example, on the inside wall of a gun tube, especially for oscillatory pressure measurements composed of high oscillatory frequencies (i.e., above 40 or $50 \mathrm{kHz}$ for the case where $c_{o}=40,000$ inches $/ \mathrm{sec}$ ).

If it is assumed that the pressure is only a function of the radial coordinate $r$, then a very conservative result (i.e., larger calculated measurement errors) is obtained for the measurement error for the maximum oscillatory pressure. Note the following when it is assumed that $p=p(\mathrm{r})$ only.

$$
\begin{aligned}
& p(t)=p_{\text {max }} \cos \omega t \\
& \omega=2 \pi f \\
& f=c_{o} / \lambda \\
& t=r / c_{o} \\
& \lambda=n d
\end{aligned}
$$

Therefore,

$$
p(r)=p_{\max } \cos \frac{2 \pi}{n d} r .
$$

The force acting on the transducer face is:

$$
\begin{aligned}
& F=\int_{A} p(r) d A=\int_{r} p(r) 2 \pi r d r \\
& F=2 \pi p_{\max } \int_{\mathrm{r}=0} r / 2 \cos \frac{2 \pi}{n d} r d r \\
& F=2 \pi p_{\max }\left\{\left(\frac{n d}{2 \pi}\right)^{2} \cos \frac{2 \pi}{n d} r+\left(\frac{n d}{2 \pi}\right) r \sin \frac{2 \pi}{n d} r\right\}_{0}^{d / 2} \\
& F=2 \pi p_{\max }\left\{\left(\frac{n d}{2 \pi}\right)^{2} \cos \frac{\pi}{n}+\left(\frac{n d}{2 \pi}\right) \frac{d}{2} \sin \frac{\pi}{n}-\left(\frac{n d}{2 \pi}\right)^{2}\right\} \\
& F=2 \pi p_{\max } \frac{n d^{2}}{4 \pi}\left\{\frac{n}{\pi}\left(-1+\cos \frac{\pi}{n}\right)+\sin \frac{\pi}{n}\right\}
\end{aligned}
$$


Consider the following special case:

$$
\begin{aligned}
& \text { let } \lambda \rightarrow \infty \\
& \therefore n \rightarrow \infty \\
& F_{\text {max }}=2 \pi p_{\max } \frac{n d^{2}}{4 \pi}\left\{\frac{n}{\pi}\left(-1+1-\left(\frac{\pi}{n}\right)^{2} \frac{1}{2}+\left(\frac{\pi}{n}\right)^{4} \frac{1}{24}-\cdots\right)+\frac{\pi}{n}-\left(\frac{\pi}{n}\right)^{3} \frac{1}{6}+\cdots\right\} \\
& F_{\text {max }}=2 \pi p_{\text {max }} \frac{n d^{2}}{4 \pi}\left\{-\frac{1}{2} \frac{\pi}{n}+\left(\frac{\pi}{n}\right)^{3} \frac{1}{24}-\cdots+\frac{\pi}{n}-\left(\frac{\pi}{n}\right)^{2} \frac{1}{6} \cdots\right\} \\
& F_{\text {max }}=2 \pi p_{\text {max }} \frac{d^{2}}{4}\left\{-\frac{1}{2}+\left(\frac{\pi}{n}\right)^{2} \frac{1}{24}-\cdots+1-\left(\frac{\pi}{n}\right) \frac{1}{6}+\cdots\right\}
\end{aligned}
$$

Since $\lambda=n d$ where $n \rightarrow \infty$, Eq. (75) results.

$$
F_{\max }=p_{\max } \frac{\pi d^{2}}{4}
$$

Dividing Eq. (74) by Eq. (75) results in Eq. (76).

$$
\begin{aligned}
& \frac{F}{F_{\max }}=\frac{2 n}{\pi}\left\{\frac{n}{\pi}\left(-1+\cos \frac{\pi}{n}\right)+\sin \frac{\pi}{n}\right\} \\
& \% \text { error }=\left[1-\frac{F}{F_{\max }}\right] \times 100
\end{aligned}
$$

or

$$
\% \text { error }=\left\{1-\frac{2 n}{\pi}\left[\frac{n}{\pi}\left(-1+\cos \frac{\pi}{n}\right)+\sin \frac{\pi}{n}\right]\right\} \times 100
$$

Table 2 lists the approximate measurement errors and associated frequencies for various values of $n$ for $c_{o}=40,000$ inches $/ \mathrm{sec}$. and $d=0.25$ inches. Note that the errors for the measured maximum oscillatory pressure are very large for this case compared to the more realistic case presented in Table 1a. 


\section{Table 2}

Measurement Error for Max. Oscillatory Pressure

Due to Frequency of Pressure Oscillation

(Pressure Wave Sweeps Across Transducer Face)

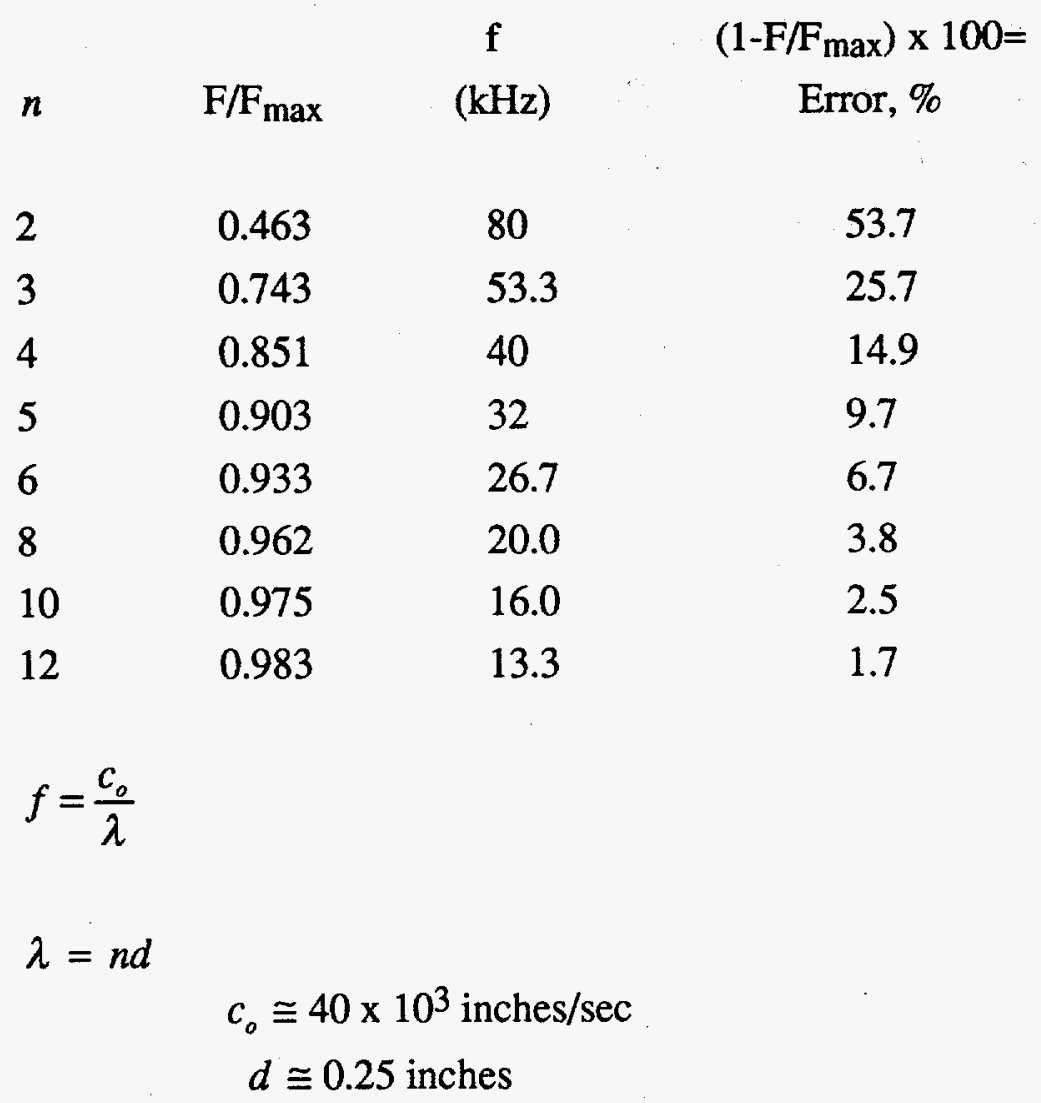

Correspondingly, if the frequency of the oscillatory pressure is $50 \mathrm{kHz}$ and the approximate error in the measured maximum oscillatory pressure is not to exceed $6.7 \%$ (i.e., $\lambda=n d$ where $n=6$ ), what is the diameter $d$ of the transducer's sensing face?

$$
\begin{aligned}
& d=\frac{c_{o}}{n f} \\
& d=\frac{40 \times 10^{3}}{6 \times 50 \times 10^{3}} \cong 0.13 \text { inches } \\
& d \cong 0.13 \text { inches }
\end{aligned}
$$




\section{REFERENCES}

1 "Dynamic Response of a Transducer Mounted at One End of an Acoustical Cavity," by G. A. Benedetti, Sandia Report SAND92-8003, April 1993.

2. "Measurement of Oscillatory Pressures Using Single Diameter and Two Diameter Transducer Cavities," by G. A. Benedetti. Presented at the JANNAF Combustion Subcommittee on "Measurement Techniques in Highly-transient, Spectrally-rich, Combustion Environments," Monterey, CA, November 19, 1993. 


\section{APPENDIX A}

\section{Pressure-Time Response Plots}

This appendix contains examples of the early and long time dynamic response of the nondimensional output pressure at $\xi=1[$ i.e., $\underset{\text { oupput }}{P(\tau)}$ vs $\tau]$ for several normalized non-dimensional specified input pressures applied at $\xi=0\left[\right.$ i.e., $\left.P_{\text {input }}(\tau) v \tau\right]$. The early time response plots, Figures A-1 through A-9, result from numerically integrating Eqs. (41) for the initial conditions given by Eqs. (42). $P(\tau)$ is then determined from Eqs. (40) and (44). Recall that the maximum value of $\underset{\text { inpu }}{ }(\tau)$ is unity and that the non-dimensional time for the pressure wave to travel the nondimensional distance $\xi=1$ is unity (refer to Figure 1 for the system configuration). The first non-dimensional undamped natural frequency for the open-closed cavity is $\theta_{1}=\frac{\pi}{2}$.

Each plot shows the input pressure $\underset{\text { input }}{P}(\tau) v s \tau$ and the output pressure $\underset{\text { ouput }}{P}(\tau) \tau$ as well as the non-dimensional physical system parameters. In each of the examples, $\tau_{1}=2.0$, and

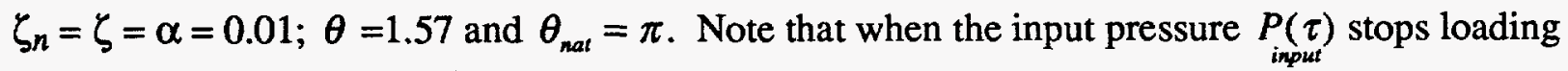
the transducer located at $\xi=1$, a damped transient oscillation for $\underset{\text { ouput }}{P(\tau)}$ occurs. Also note that for these calculations, it is assumed that the non-dimensional cavity pressure at static equilibrium (or the initial non-dimensional cavity pressure) $\frac{p_{o}}{\rho_{o} c_{o}^{2}}$ is sufficiently large such that the minimum non-dimensional absolute output pressure $\frac{p(1, \tau)}{\rho_{o} c_{o}^{2}}$ is not less than zero. For example, in Figure A-9, if $\frac{p_{o}}{\rho_{o} c_{o}^{2}} \geq 20$, then $\left|\frac{p(1, \tau)}{\rho_{o} c_{o}^{2}}\right|_{\text {min }} \geq 0$. Refer to Eq. (52). Consequently, the non-dimensional mean pressure $\frac{p_{o}}{\rho_{o} c_{o}^{2}}$ is not included in the figures in this appendix.

On Figures A-9 and A-10, the horizontal line for $P(\tau)=14.5$ represents the maximum steady state value of $\underset{\text { ouput }}{P(\tau)}$ where $P(\tau)=\sin \theta \tau$ for $0 \leq \tau \leq \infty$ and $\zeta_{n}=0$ for all values of $n$. Note that $\theta \cong \frac{\pi}{2}$. Refer to reference 1, Eq. (53). Note also that for $\tau=210$, steady state vibration has not yet occurred. Steady state vibration occurs when $\tau>650$ (refer to Figure A10). When $\zeta_{n}=\zeta=0.01$, the transient dynamic response will damp out slowly compared to the case where $\zeta_{n}$ is much larger than 0.01 . Since the transient dynamic response, which consists of 
oscillations at the damped natural frequencies, damps out or disappears as $\tau$ increases indefinitely, the dynamic response of the system becomes a steady state harmonic oscillation of non-dimensional circular frequency $\theta$ and the amplitude of the steady state oscillation depends on the non-dimensional physical system parameters. For the case where $\zeta_{n}=0$ and $\zeta=\alpha=0.01$ (steady state amplitude of dimensionless pressure equal to 14.5), the transient dynamic response damps out very slowly (refer to Figure A-10). That is, there is only damping in the transducer and no damping in the fluid (or gas). The amplitude modulated transient response (many "lobes") results because Eqs. (41) are highly coupled (i.e., $\alpha=0.01$ and $\theta_{\text {nat }}=\pi$ ) and many of the lower modal frequencies differ only slightly from each other. Consequently, during the slightly damped transient vibration, energy is transferred between the lower modes and this is observed in Figure A-10 as an amplitude modulated transient vibration.

When Eqs. (46) are numerically integrated and the solution vectors are used to determine $\underset{\text { ouput }}{P(\tau)}$ for the case where $\zeta_{n}=0.01$ and $\theta=1.57$, Figure A-11 results. Recall that this is the "simplified boundary condition" case and the non-dimensional physical system parameters $\zeta, \alpha$, and $\theta_{\text {nat }}$ are not applicable for this special case. $P(\tau)$ in is the same as in Figure A-10.

Figure A-12 represents the case where $\zeta_{n}=\zeta=\alpha=0.01, \theta_{\text {nat }}=\pi$ and $\underset{\text { input }}{P(\tau)}=\sin \theta \tau$ where $\theta=0.1 \pi$. Note that in this case $\theta_{\text {nat }} \gg \theta_{1}$, and $\theta_{1} \gg \theta$. Recall that $\theta_{1}=\frac{\pi}{2}$.

Figure A-13 represents the case where $\zeta=0.01$ and $P_{\text {input }}(\tau)=\sin \theta \tau$ where $\theta=0.1 \pi$. For this case, $\theta_{1} \gg \theta$ and $\zeta_{n}, \alpha$, and $\theta_{\text {nat }}$ are not applicable since Eqs. (46) are numerically integrated (i.e., simplified boundary conditions).

Figure A-14 is similar to Figure A-12, only in Figure A-14 $\zeta_{n}=\zeta=0.05$ and $\alpha=0.01$.

Figure A-15 is similar to Figure A-13, only in Figure A-15 $\zeta=0.05$.

In Figures A-12 through A-15, both $\underset{\text { input }}{P(\tau)}$ and $\underset{\text { oupput }}{P(\tau)}$ are plotted on each plot. 


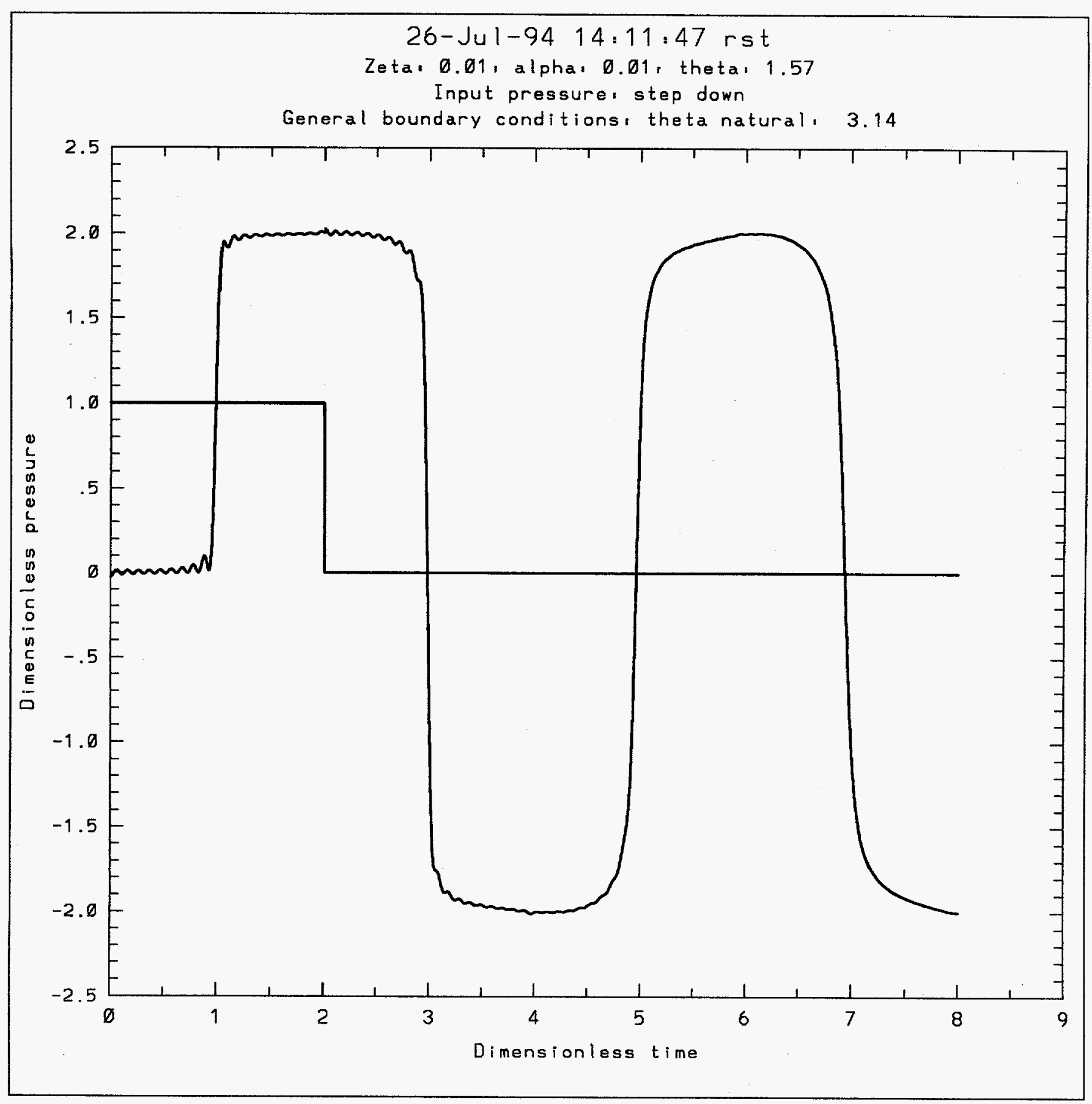

Figure A-1 


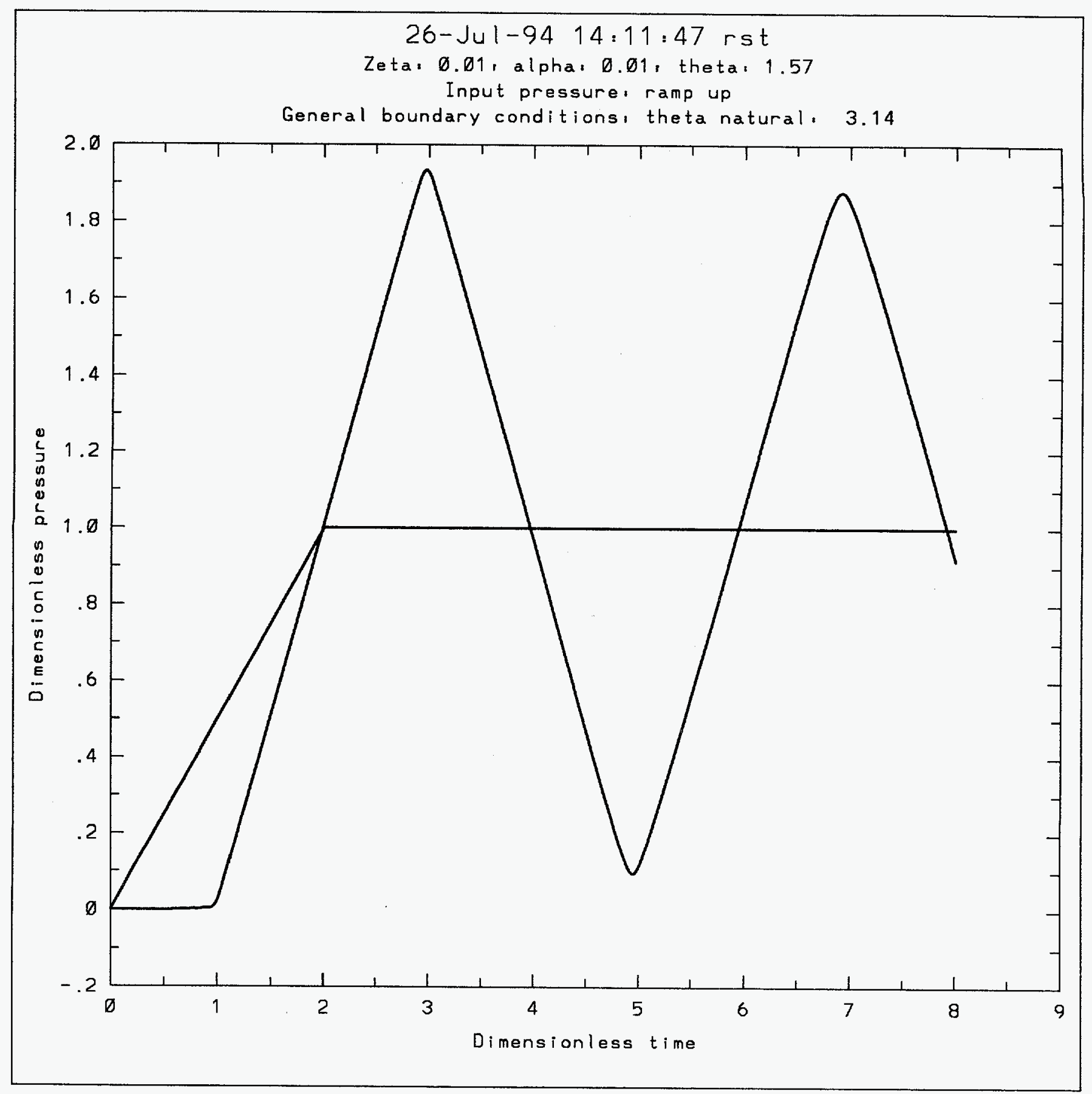

Figure A-2 


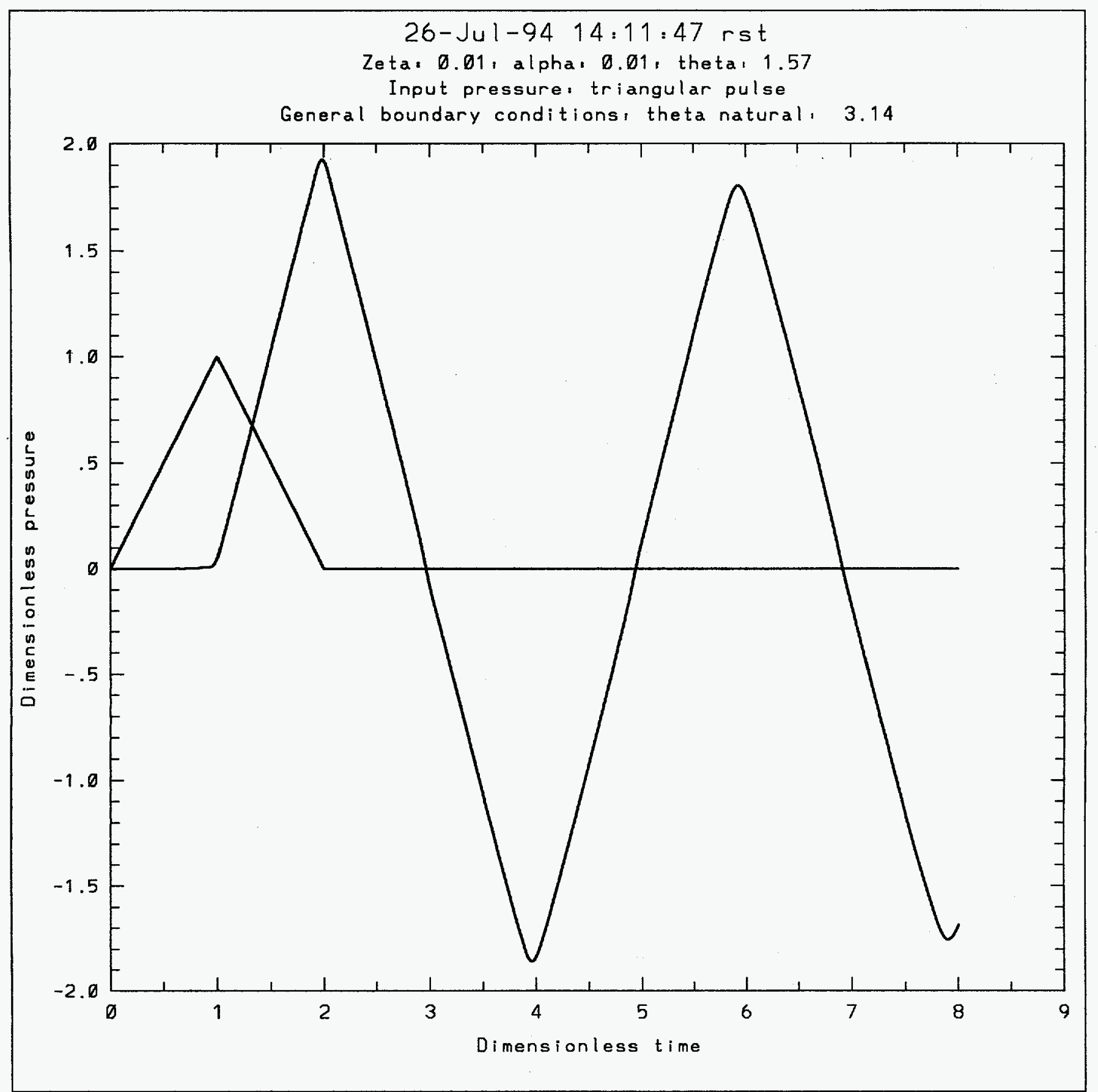

Figure A-3 


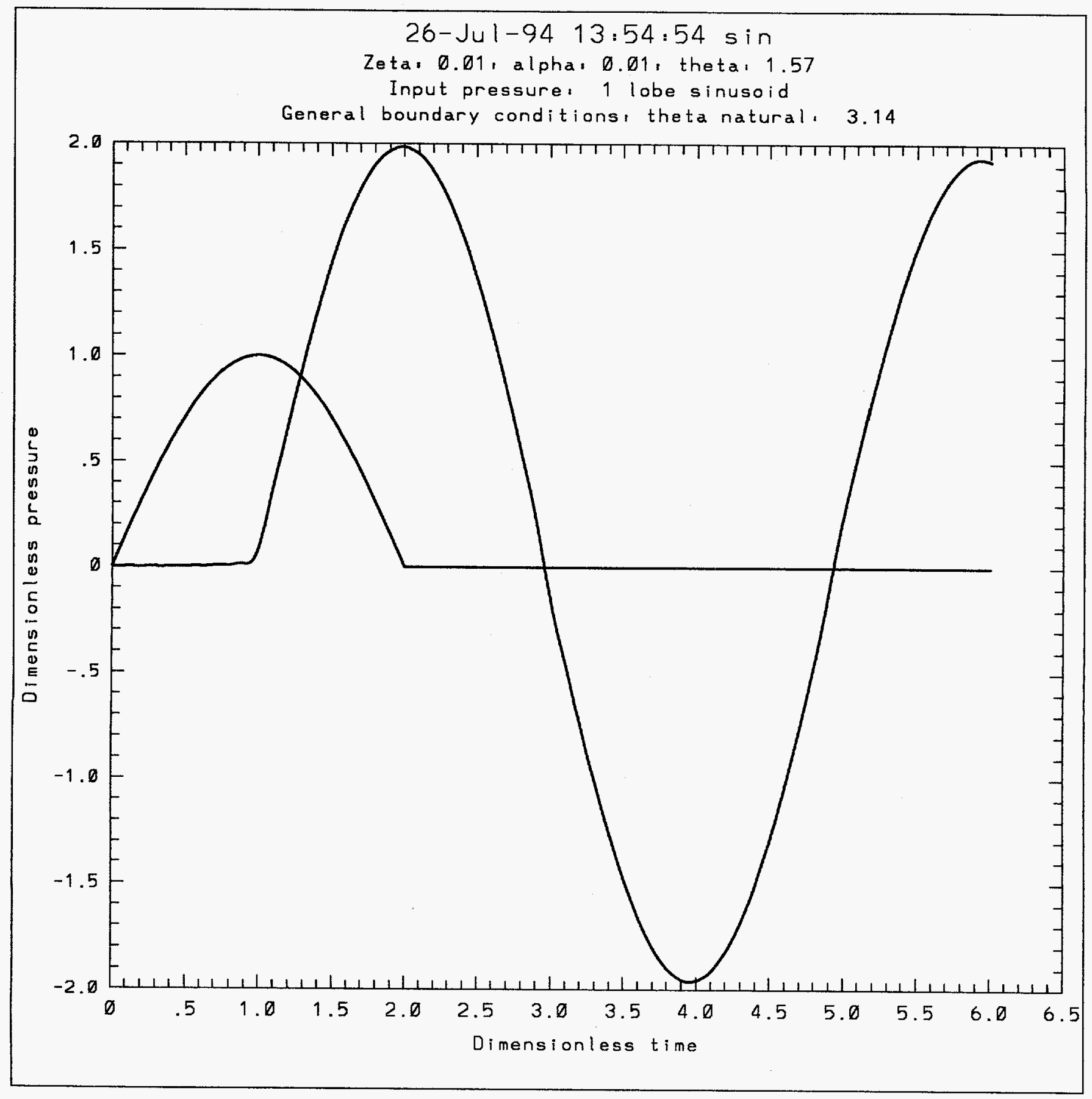

Figure A-4 


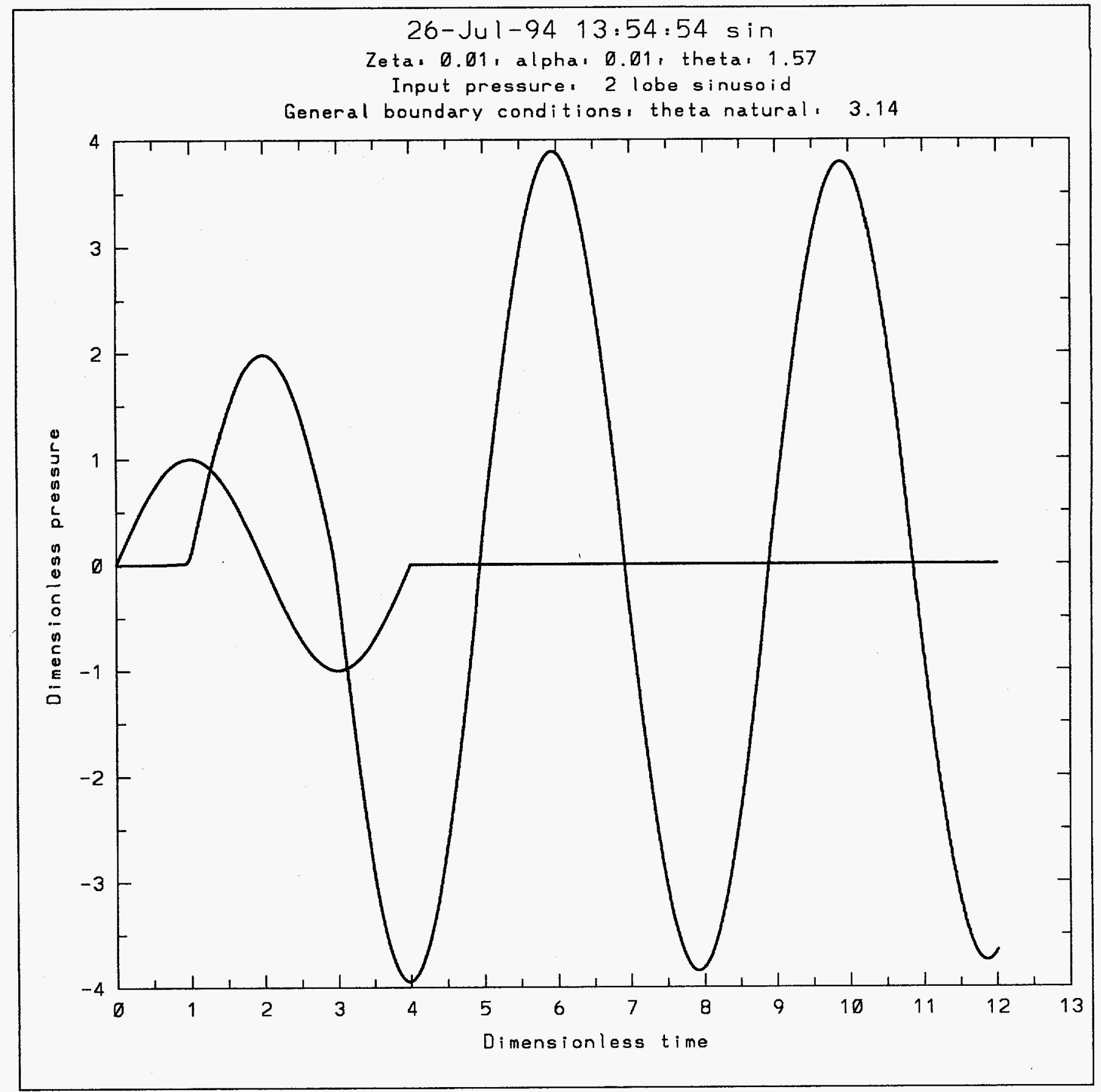

Figure A-5 


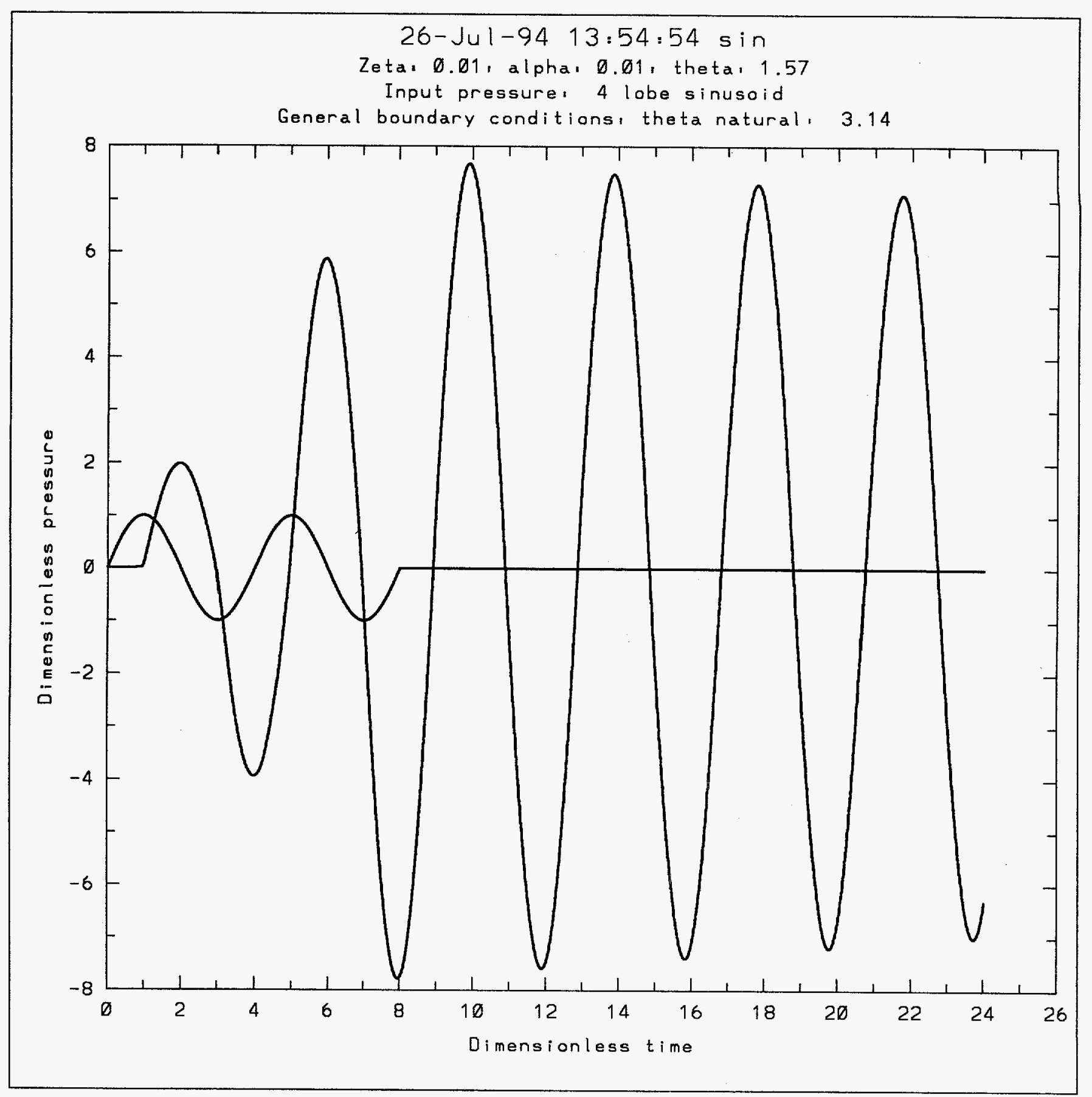

Figure A-6 


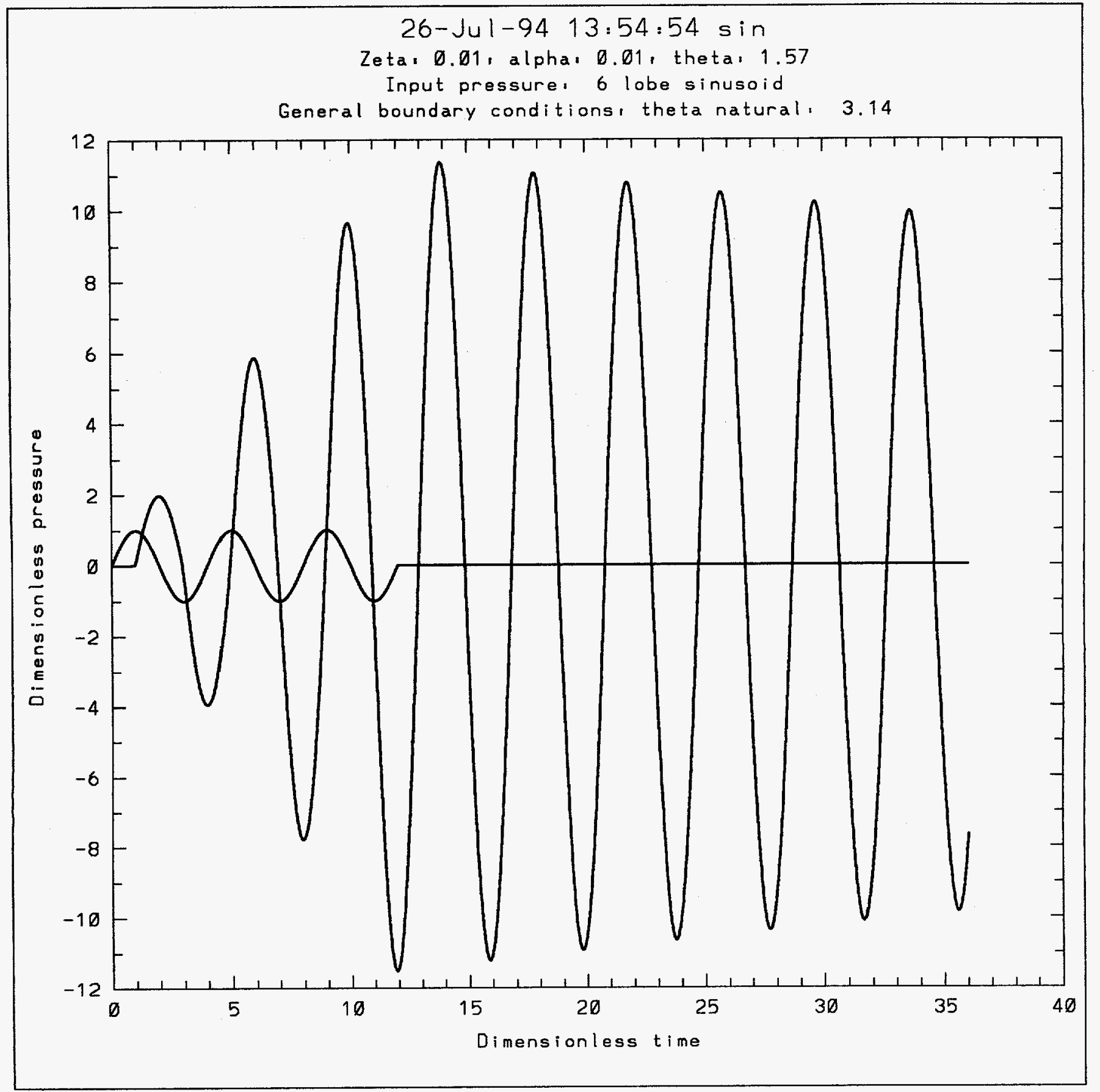

Figure A-7 


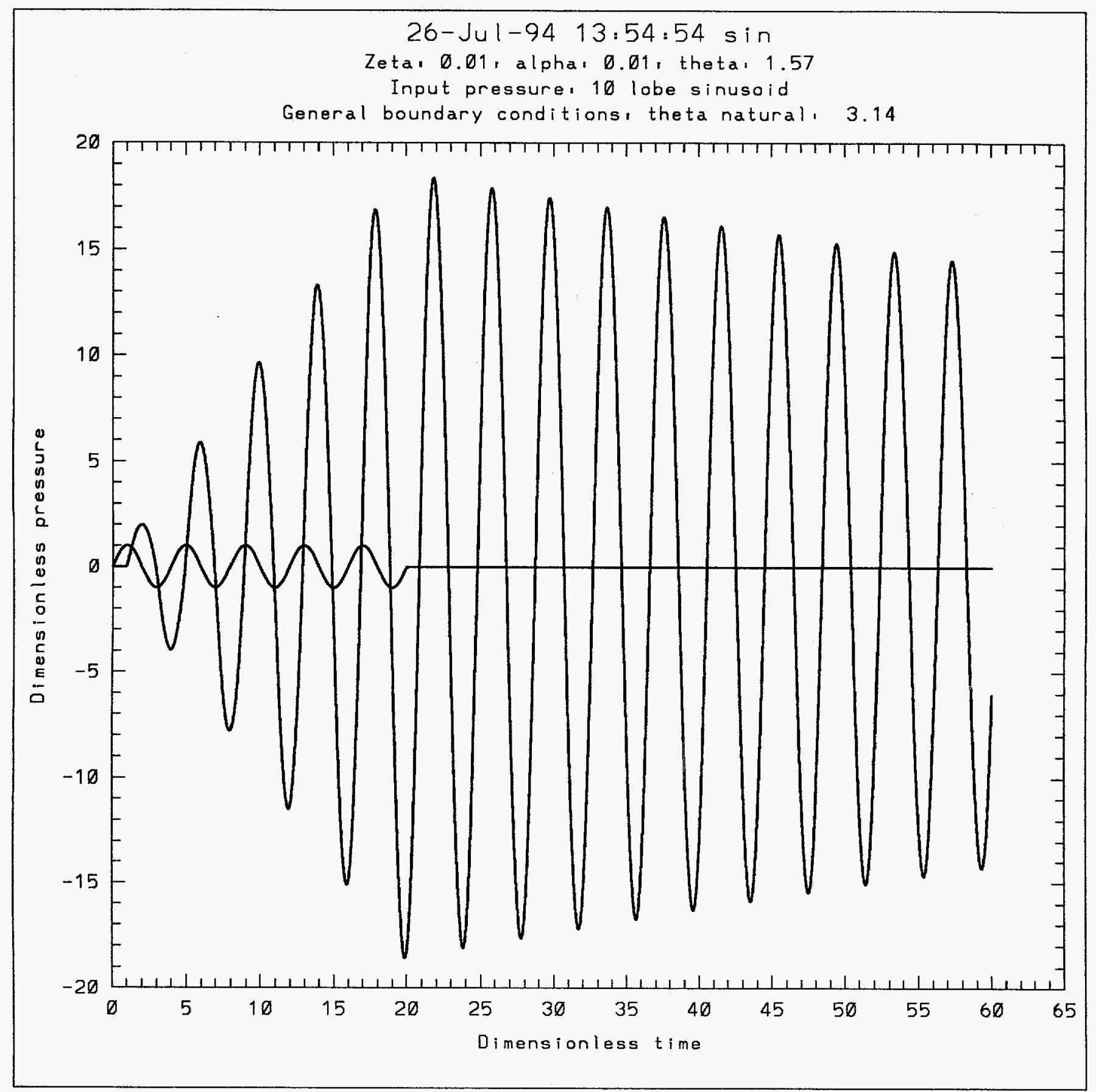

Figure A-8 


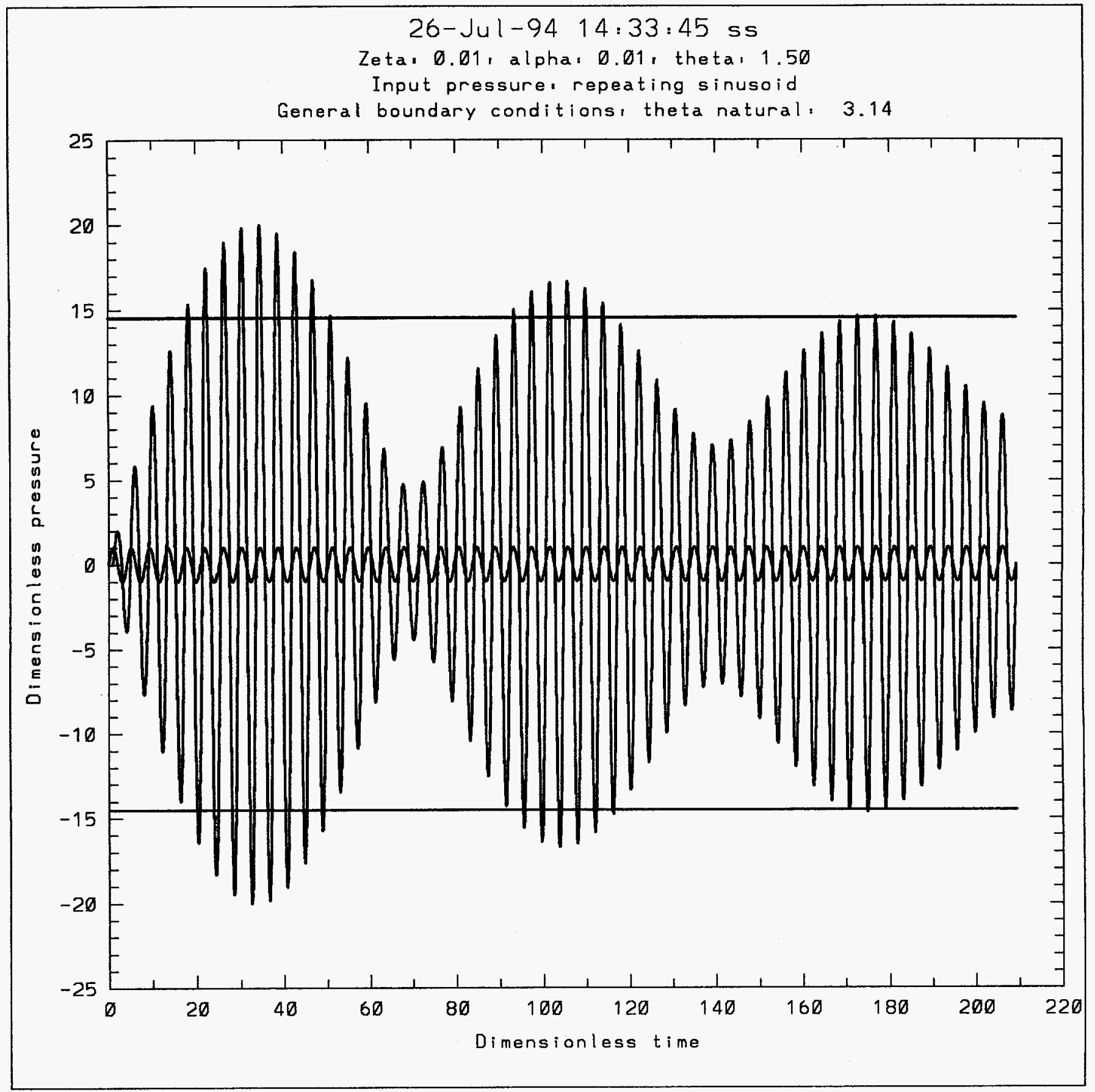

Figure A-9 


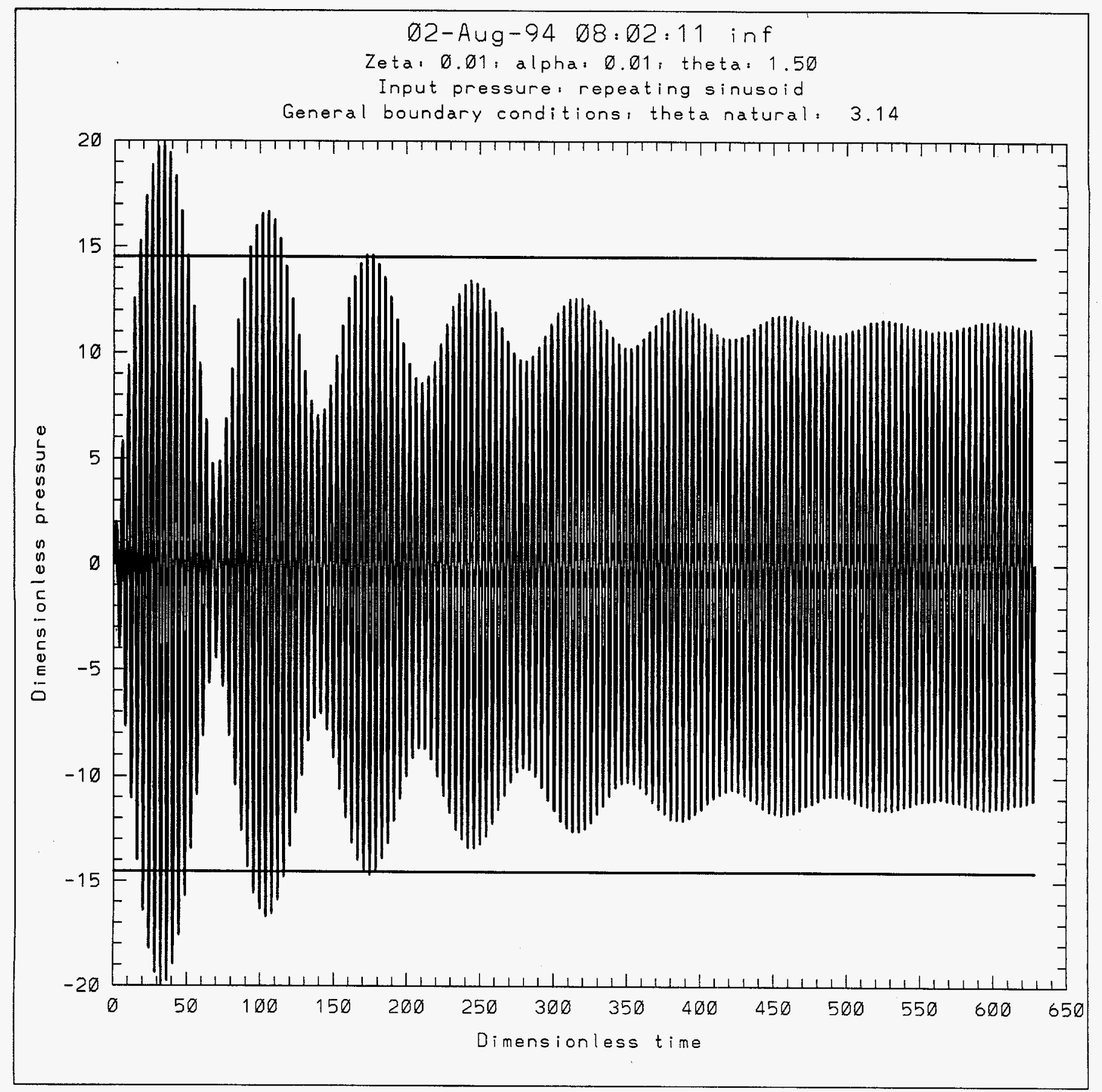

Figure A-10 


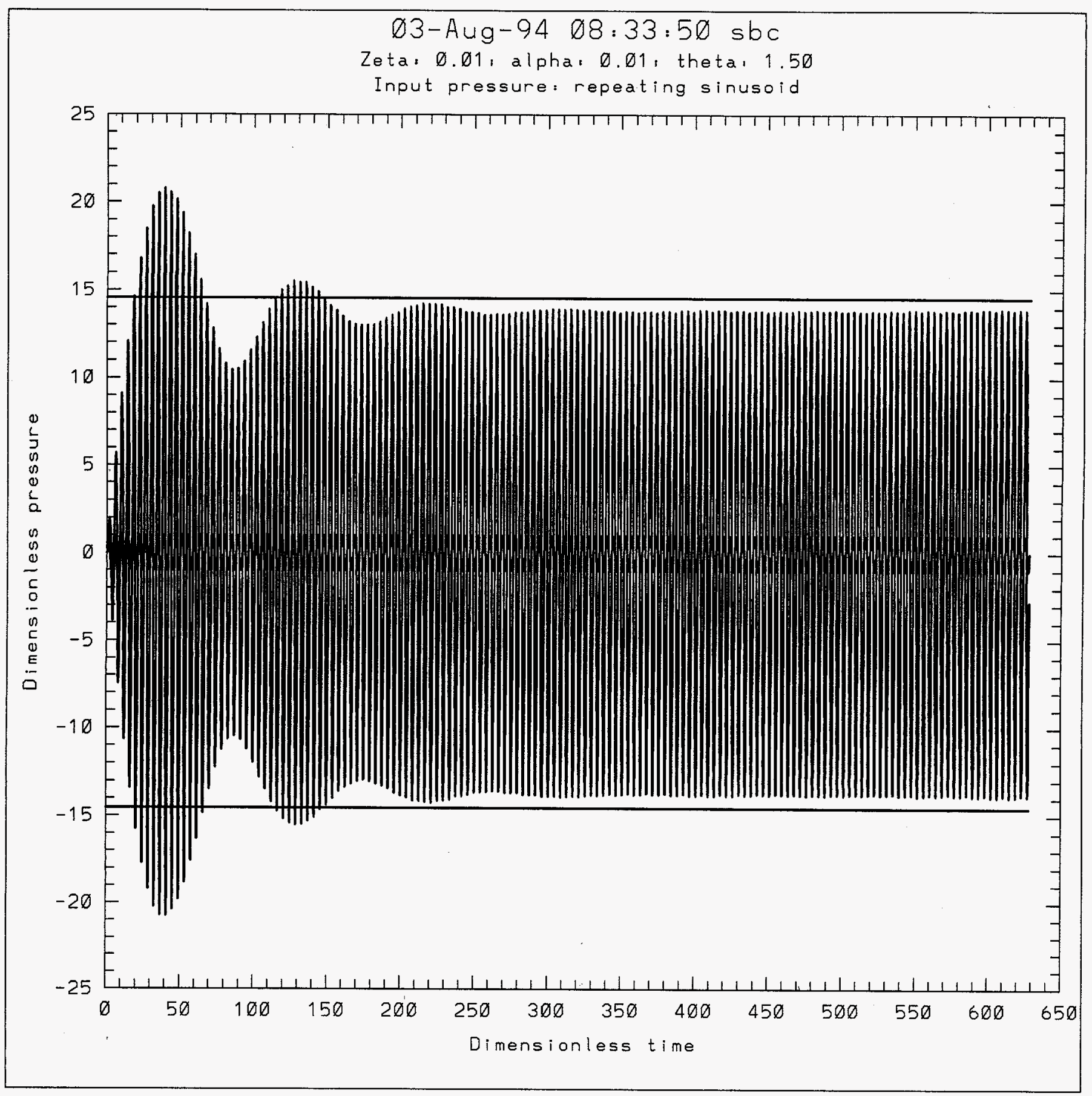

Figure A-11 


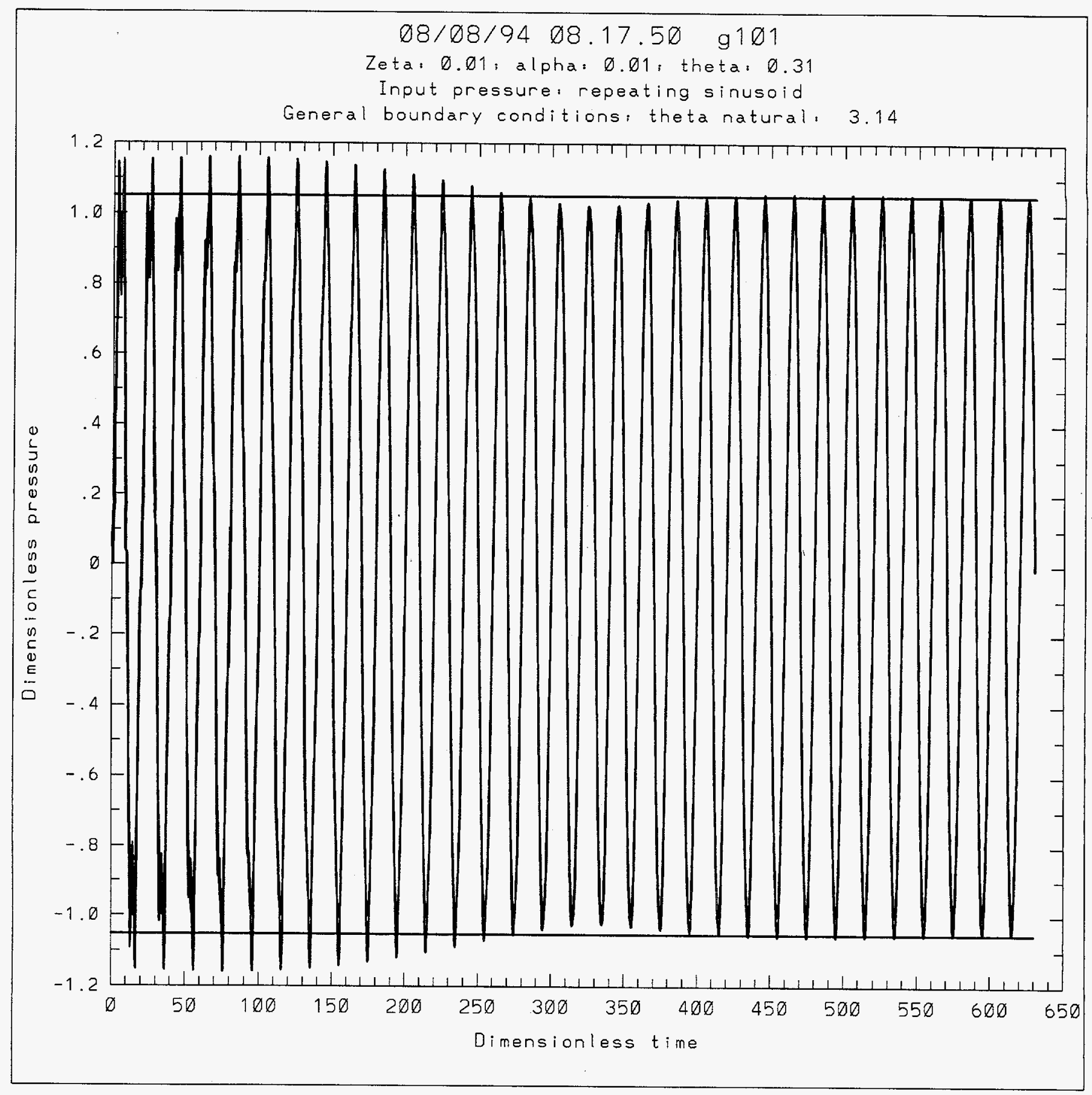

Figure A-12 


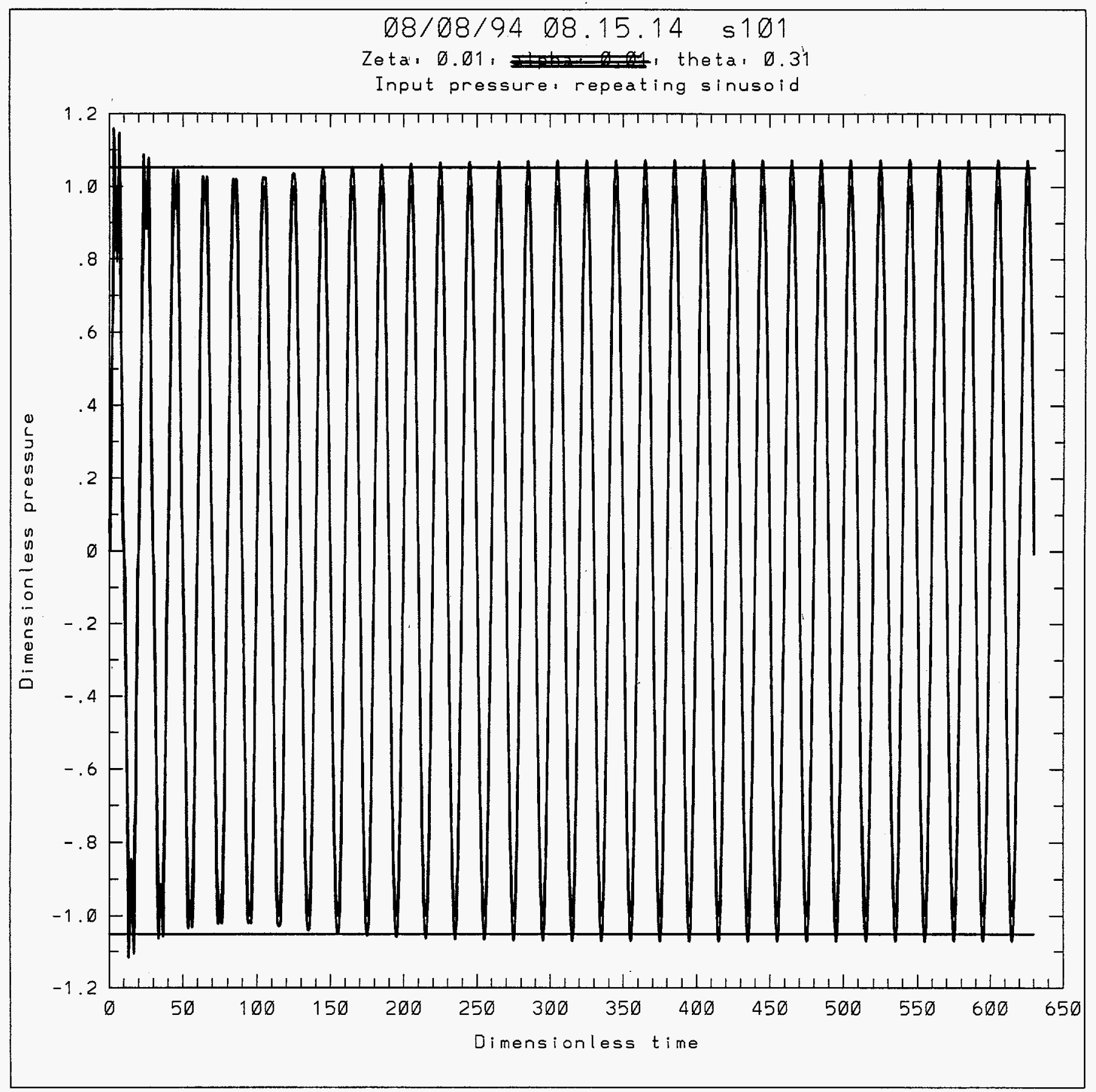

Figure A-13 


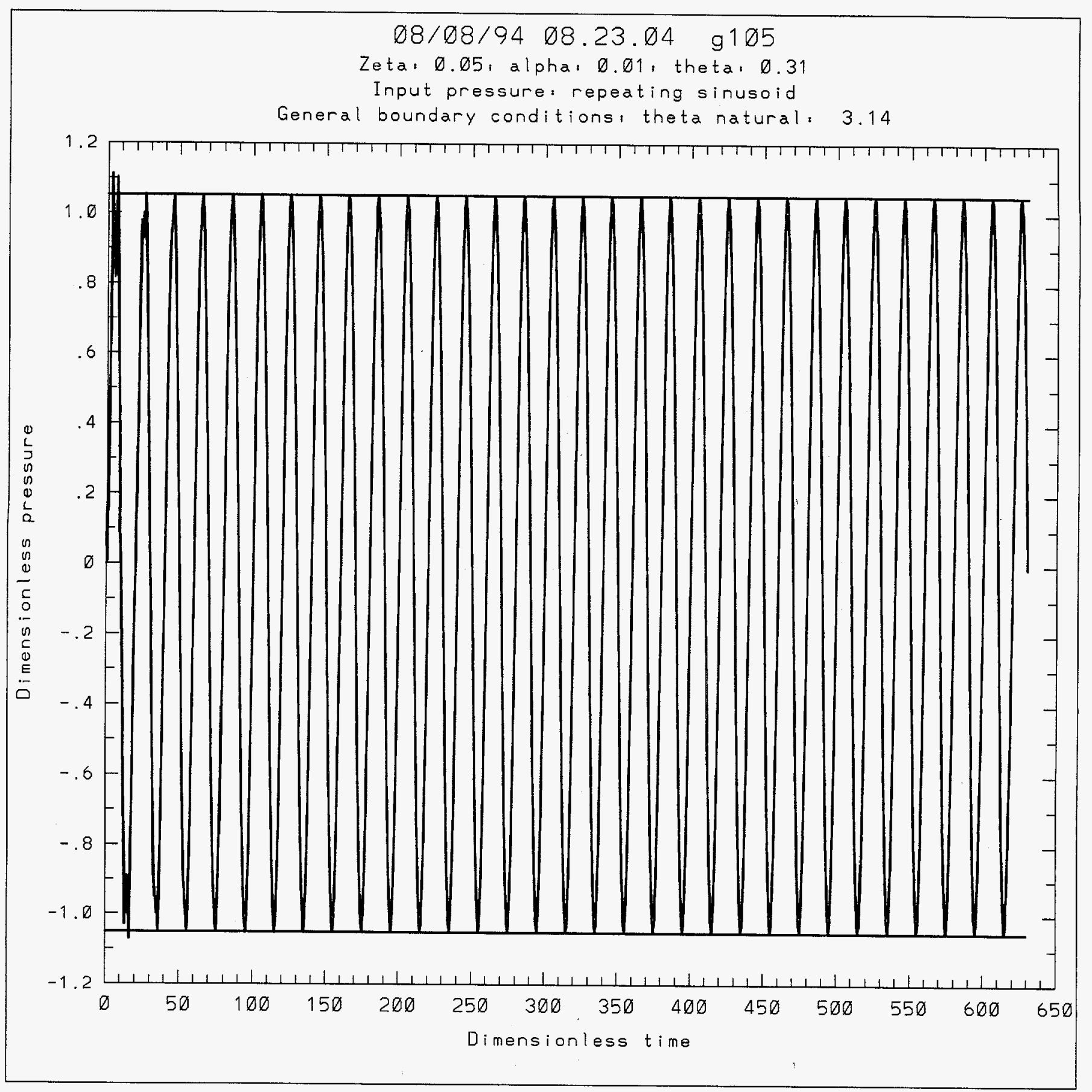

Figure A-14 


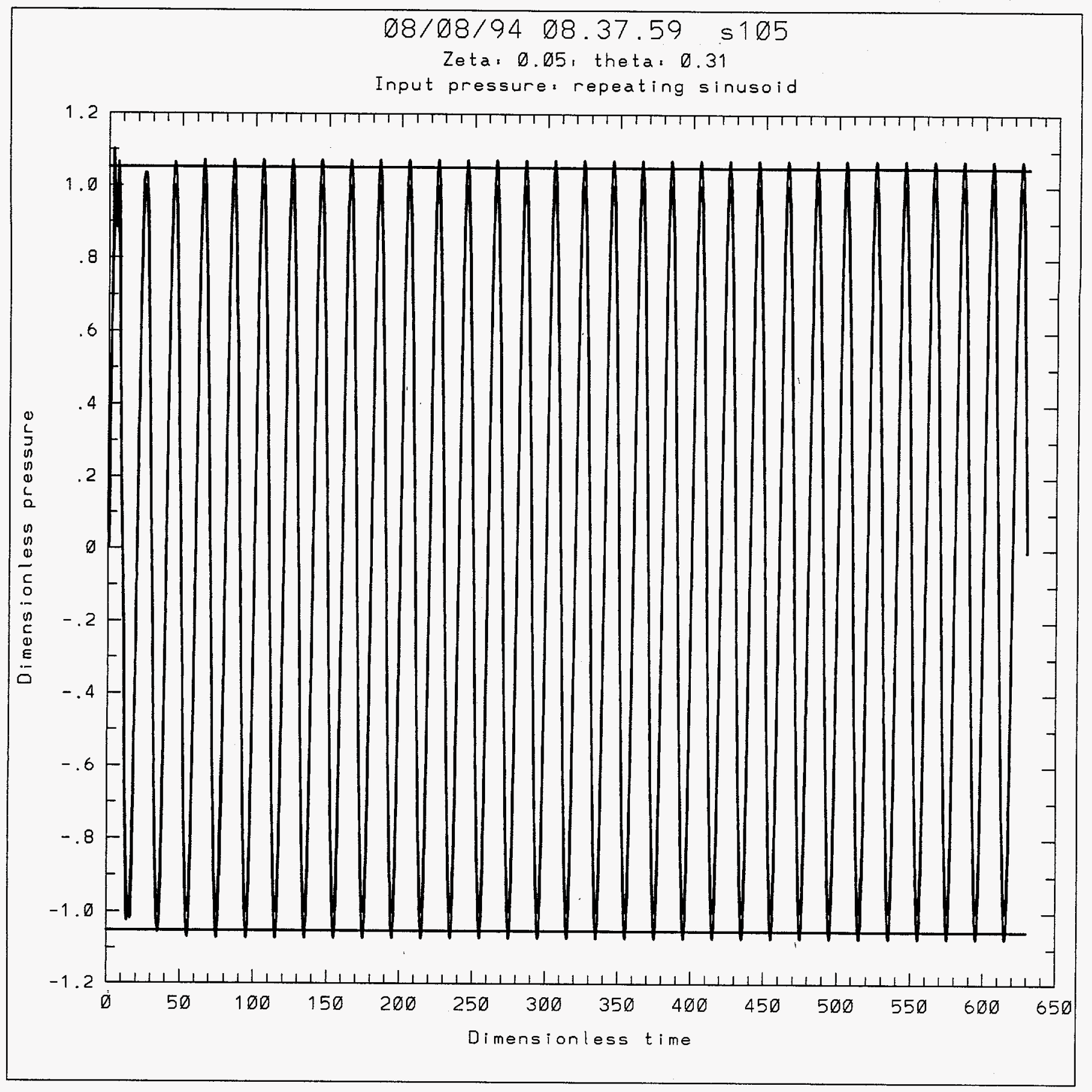

Figure A-15 
UNLIMITED RELEASE

\section{INITIAL DISTRIBUTION}

U.S. Army Research Laboratory (15)

Attn: Director

Kenneth Bannister, AMSRL-WT-PD

Bruce Burns, AMSRL-WT-PD (3)

James DeSpirito, AMSRL-WT-PA

William Drysdale, AMSRL-WT-PD

Thomas Erline, AMSRL-WT-PD

Al Horst, AMSRL-WT-PA

Mark Kregel, AMSRL-WT-PC

Ingo May, AMSRL-WT-P

Thomas C. Minor, AMSRL-WT-PA

Walter Morrison, AMSRL-WT-T

Todd Rosenberger, AMSRL-WT-PE

Gloria P. Wren, AMSRL-WT-PA

Aberdeen Proving Ground, MD 21005-5066

U.S. Army R\&D Engineering Center (13)

Attn: Commander

Carmine J. Spinelli, SMCAR-TD

Project Manager, Office for Advanced

Field Artillery Systems, OPM-AFAS

K. Chung, SMCAR-FSN-N (2)

Lt. Col. D. Ellis, OPM-AFAS

Richard Kopmann, SMCAR-FSS-DH

Ted Kuriata, OPM-AFAS

Barbara Machak, SMCAR-FSS- A

Dave Menchala, SMCAR-FSS-A

Jim Shields, OPM-AFAS

Susan Traendly, SMCAR-FSS-A (2)

Picatinny Arsenal, NJ 07806-5000

Los Alamos National Laboratory (2)

Attn: Tom Butler

MEE-13, MS J576

Don Rabern

P.O. Box 1663

Los Alamos, NM 87545

Martin Marietta

Attn: William Sliney

100 Plastics Avenue

Pittsfield, MA 01201

PCB Piezotronics, Inc. (2)

Attn: Dave Jaros

3425 Walden Avenue

Depew, NY 14043 
0419 J. D. Rogers, 4112

0437 K. W. Schuler, 1518

0482 A. E. Hodapp, 1502

0744 T. G. Priddy, 6448

0763 W. F. Hartman, 9614

0836 C. W. Peterson, 1516

0841 D. J. McCloskey, 1500

0953 W. E. Alzheimer, 2900

1007 A. T. Jones, 2172

1375 L. D. Bertholf, 4400

9001 J. C. Crawford, 8000

Attn: R. J. Detry, 8200

W. J. McLean, 8300

L. A. Hiles, 8400

L. A. West, 8600

T. M. Dyer, 8800

9005 J. B. Wright, 5300

Attn: C. A. Pura, 5362

D. J. Beyer, 5363

R. D. Monson, 5364

C. T. Story, 5365

R. G. Miller, 5366

9004 M. E. John, 8100

9005 W. G. Wilson, 5304

9006 E. E. Ives, 5200

Attn: D. J. Bohrer, 5202

9036 W. Bolton, 8102

9053 C. M. Hartwig, 8366

9042 G. A. Benedetti, 8741 (3)

9042 J. Z. Benson, 8741

9042 M. L. Chiesa, 8741

9042 S. K. Griffiths, 8745

9042 Y-R Kan, 8741

9042 J. C. Keilman, 8741

9042 F. L. Rasmussen, 8741

9043 P. E. Nielan, 8742 (2)

9201 R. J. Gallagher, 8114

Attn: M. Abrams, 8114

9401 R. C. Wayne, 8700

Attn: M. L. Callabresi, 8743

R. J. Kee, 8745

W. A. Kawahara, 8746

9401 C. W. Robinson, 8702

9406 H. H. Hirano, 8412

9407 E. T. Cull, 8415

9731 D. B. Nelson, 8283

9022 Mail Distribution Team, 8533-1 for OSTI (10)

9022 Mail Distribution Team, 8533-1/Technical Library, MS8099, 13414

8099 Technical Library 13414 (4)

9018 Central Technical Files, 8523-2 (3) 